KNOWLEDGE TRANSLATION IN ACTION:

\title{
AN EVALUATION OF THE IMPLEMENTATION OF CUSTOMISED BIRTHWEIGHT CENTILES AT ONE NEW ZEALAND DISTRICT HEALTH BOARD
}

by

Malia Noelani Lardelli

A thesis submitted to the Victoria University of Wellington in fulfilment of the requirements for the degree of Master of Midwifery

Victoria University of Wellington 2011 


\begin{abstract}
Identifying small for gestational age (SGA) and large for gestational age (LGA) babies is important, because these babies may be at increased risk of hypoglycaemia at birth. It is proposed that customised birthweight centiles (CBWC) can more accurately identify these babies by taking into account several physiological variables of the pregnancy affecting birthweight. These variables are: maternal height and booking weight, ethnicity, parity, the baby's gender and gestation. CBWC for a New Zealand (NZ) population were developed by McCowan, Stewart, Francis and Gardosi (2004), and can be downloaded for free from www.gestation.net. Using CBWC is not in common practice in NZ, even though its use was discussed in a 2007 Health and Disability Commissioner report in relation to neonatal hypoglycaemia. The setting for the study was a NZ District Health Board (DHB) that calculates a CBWC for each birth. The research question asked how the evidence behind CBWC was put into practice and what outcomes resulted from translating this knowledge into action. This was a mixed methods evaluation which included interviews, focus groups, an audit and document analysis. The knowledge-to-action (KTA) framework (Straus, Tetroe, \& Graham, 2009) was used as a theoretical template to describe the implementation process that occurred. The results revealed that maternity care providers were initially challenged by the evidence. But over time, guideline compliance improved as practitioners experienced the benefit of using CBWC in practice. All agreed that the CBWC calculator was user-friendly. However, the audit demonstrated it was easy to make a mistake or manipulate results when using the calculator. CBWC can help identify babies at risk of hypoglycaemia who otherwise would have been missed. But it is unknown what difference it had made in improving neonatal morbidity and mortality, due to insufficient data. But anecdotally, stakeholders felt it had made a difference. The findings demonstrated that knowledge translation is a complex process which is difficult to capture within a one-dimensional framework. However, using such a framework can identify what stages are needed to complete an implementation process.
\end{abstract}


Key words - customised birthweight centiles, knowledge translation, small for gestational age, large for gestational age, evaluation, mixed methods. 


\section{Acknowledgements}

I wish to sincerely thank all those who supported me through this Master's Thesis journey.

Thank you to everyone at the DHB who participated in this study. I couldn't have done it without your willingness to be involved. I'd especially like to thank the Midwifery Educator for all her help and the manager and staff of the clinical records department who located over 600 clinical notes for me!

I would like to thank the Faculty of Humanities and Social Sciences for the funding provided to undertake the fieldwork associated with this research. I would also like to thank the University for the Tu Horomata Scholarship received, which is intended to retain and upskill Maori at a Masters level. Finally, I would also like to thank Health Work Force NZ for a postgraduate midwifery grant which assisted with paying course fees.

A special acknowledgement needs to be made to my supervisor Dr Joan Skinner for keeping me focused and her continued enthusiasm and encouragement. Thank you also to the Graduate School of Nursing, Midwifery and Health including the support staff for all your help. Another big thank you also to the assistance provided by the library distance learning service. Although I have never met any of you I cannot thank you enough for the amazing service you provide.

Thank you to mum for assisting to edit my Thesis. A special thanks to my husband Jason for all his love, support and patience. Finally, I'd like to dedicate this thesis to Samuel and Noelani. I hope that you will both develop a love of learning like your Mummy and I look forward to having more time to spend together as a family. 


\section{Table of Contents}

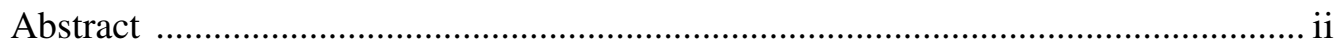

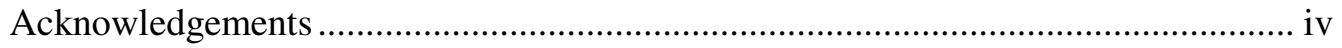

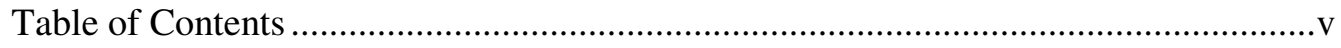

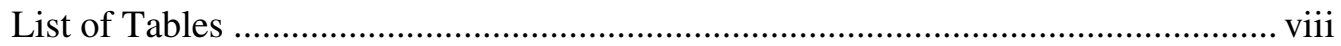

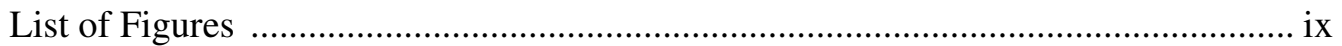

Chapter One: Introduction ...................................................................... 1

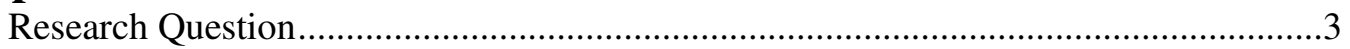

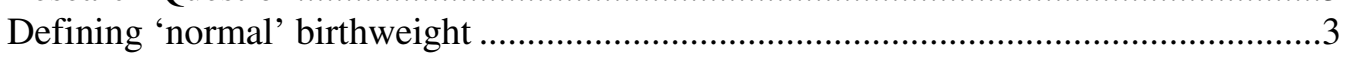

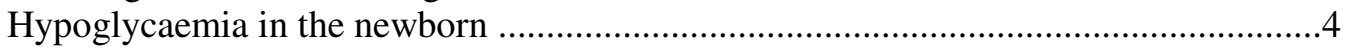

The Health and Disability Case (Case 05HDC16723) ................................................6

Better identifying babies at risk of hypoglycaemia due to their birthweight ................. 7

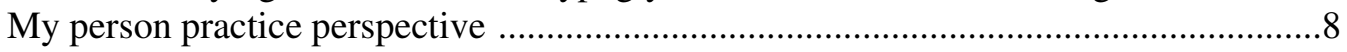

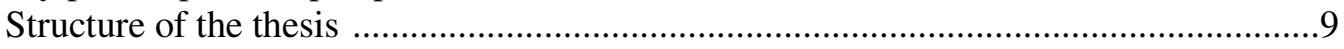

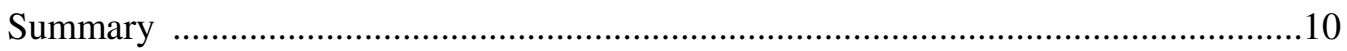

Chapter Two: Customised Birthweight Centiles ................................. 11

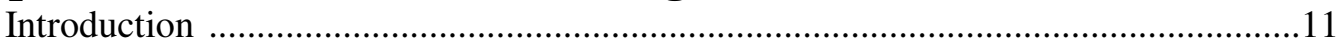

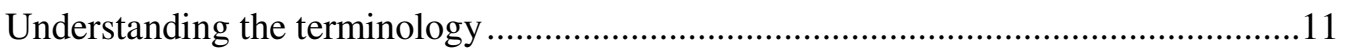

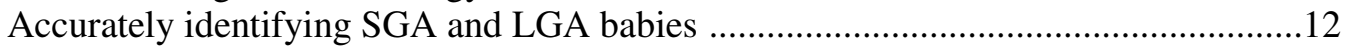

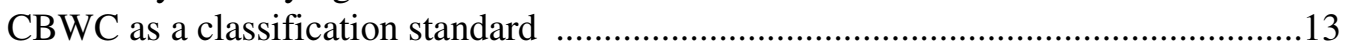

The development of 'GROW' (Gestation Related Optimum Weight) ..........................15

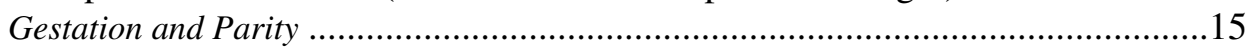

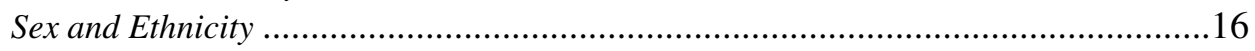

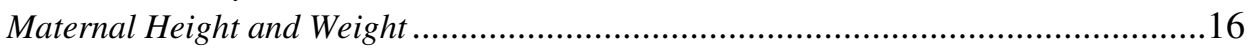

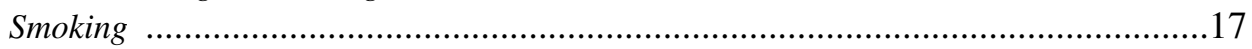

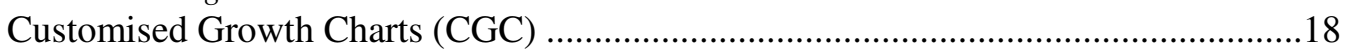

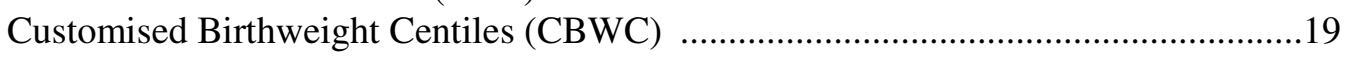

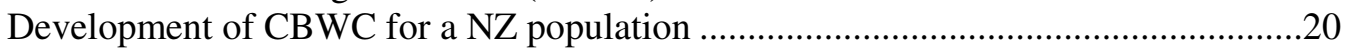

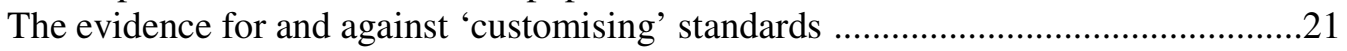

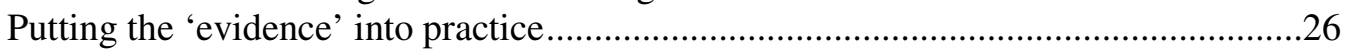

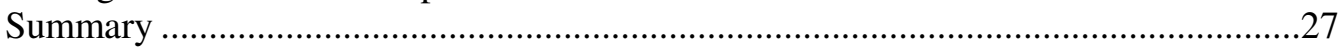

Chapter Three: Knowledge Translation ............................................... 28

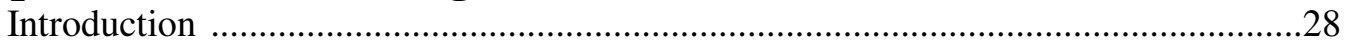

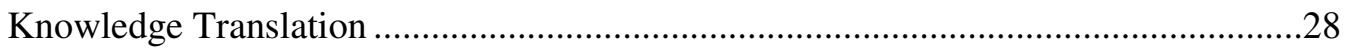

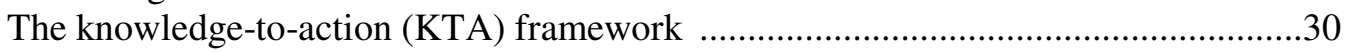

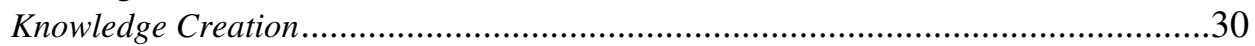

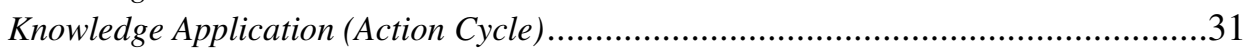

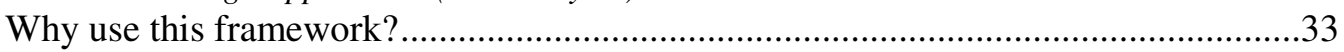

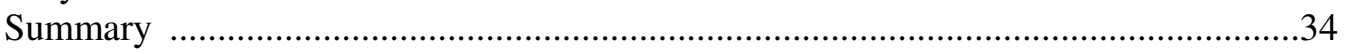

Chapter Four: Research Approach and Design................................. 35

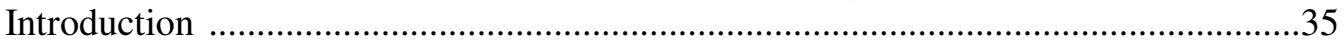

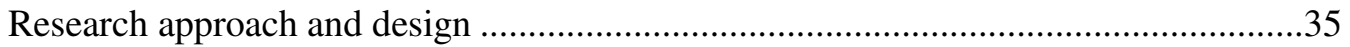

The value in using a mixed method evaluation approach ............................................37 


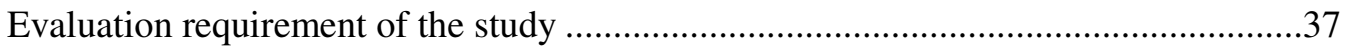

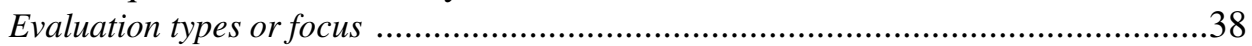

Applying a mixed method evaluation approach .......................................................39

Using the KTA framework as a research tool ........................................................4

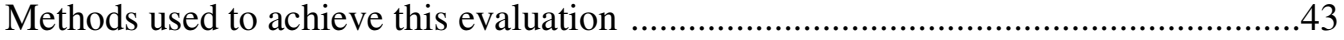

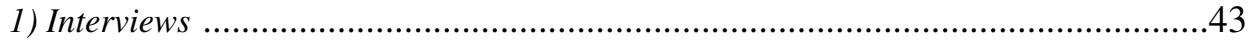

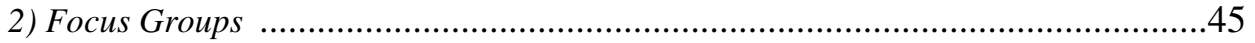

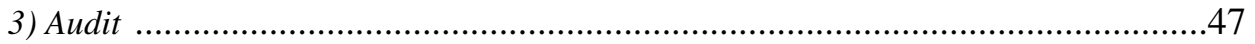

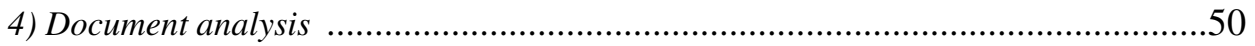

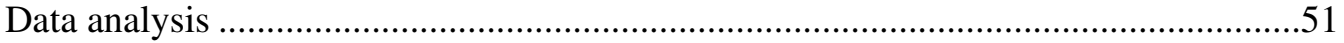

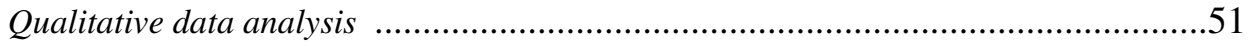

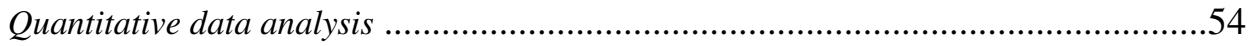

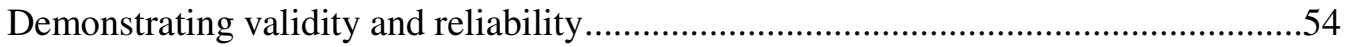

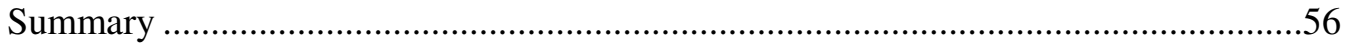

Chapter Five: Results .....................................................................5 57

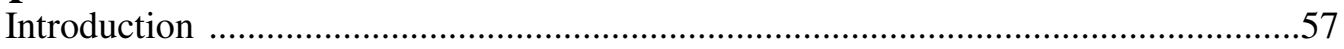

Part A: The implementation process, the story begins ............................................57

1) Identify problem $\leftrightarrow$ Identify, review, select knowledge ....................................58

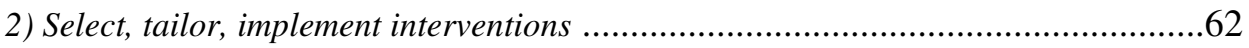

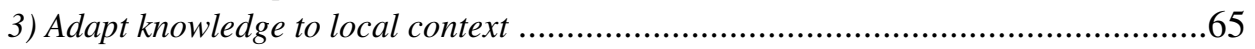

4) Assess barriers to knowledge use ....................................................................66

Part B: Measuring the extent of implementation, the story continues .....................73

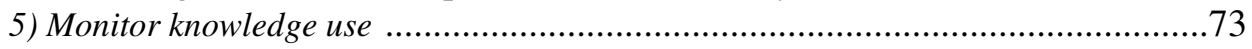

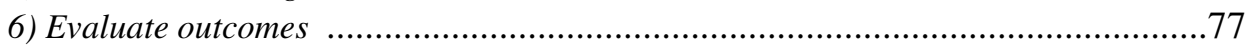

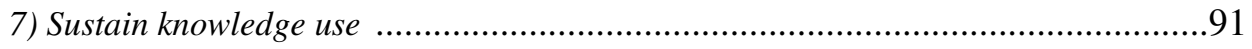

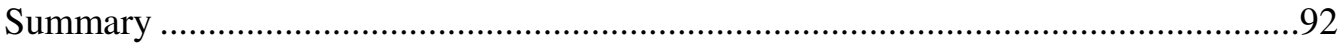

Chapter Six: Discussion ........................................................................ 94

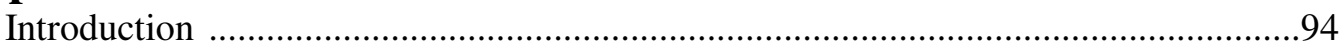

Section A: The process of implementing CBWC ...................................................94

1) Accuracy of the CBWC result: It's easy to use, however it's easy to make a mistake 94

2) Practice improved over time: You have to experience the benefit, before you believe

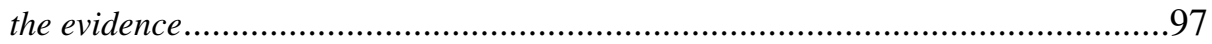

3) Did it make a difference?: Was a case similar to the HDC scenario prevented?.......99

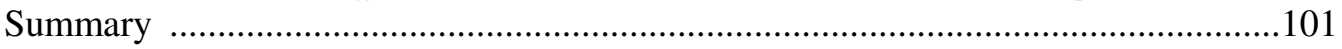

Section B: The knowledge translation framework ...................................................102

1) The KTA framework is unnecessarily complex; however, knowledge

translation is a complex process ......................................................................102

2) Knowledge translation can happen without a framework, but using the KTA

framework can help to complete the process ......................................................105

3) A champion facilitates the knowledge translation process ..................................106

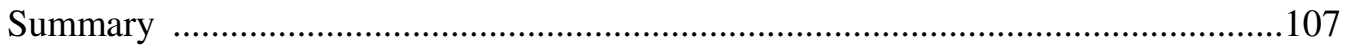

Section C: Reflections on the research as a whole ................................................107

1) It was not possible to do a process-outcome evaluation ......................................107

2) Using CBWC facilitated a proactive approach to managing hypoglycaemia and made practitioners more aware of the bigger picture of risk assessment ..........................108

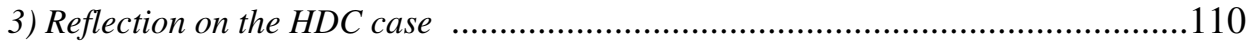

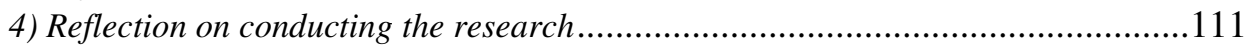

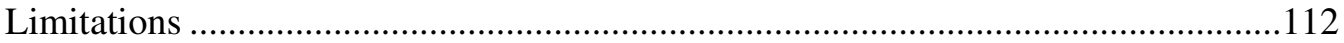


Section D: Significance/Recommendations/Ideas for further research

1) Significance

2) Recommendations

3) Ideas for further research

Conclusion: Revisiting the Research Question .................................... 117

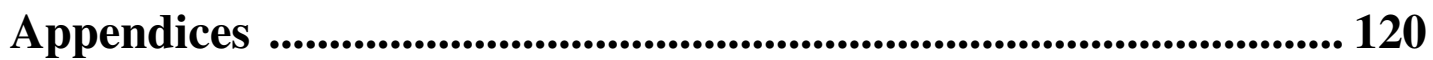

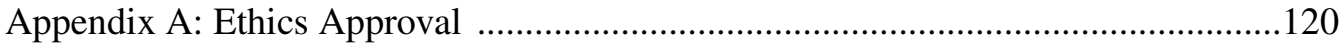

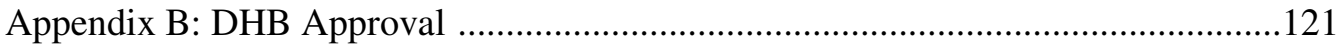

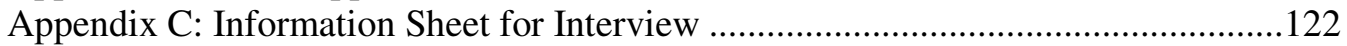

Appendix D: Consent Form for Interview ..........................................................124

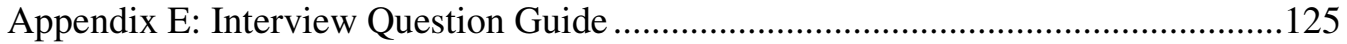

Appendix F: Information Sheet for Focus Group ................................................126

Appendix G: Consent Form for Focus Group ........................................................128

Appendix H: Invitation Poster for Focus Group .......................................................129

Appendix I: Focus Group Question Guide LMCs ...................................................130

Appendix J: Focus Group Question Guide Core Staff ...............................................131

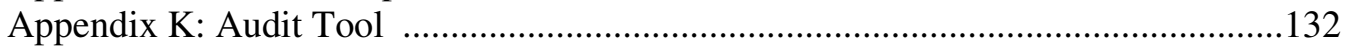

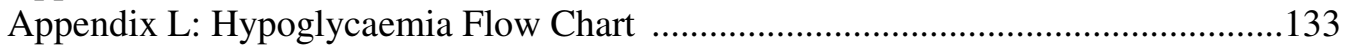

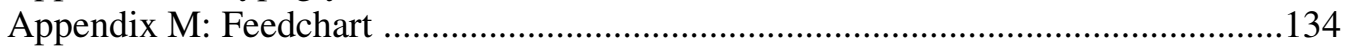

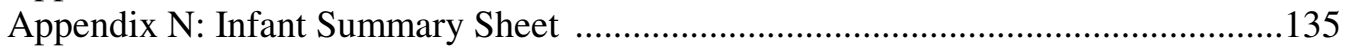

Appendix O: Population Birthweight Centile Charts ..................................................136

References ............................................................................................... 138 


\section{List of Tables}

Table 1. Studies providing the evidence for and against using 'customised' standards verses population based standards

Table 2. Timeline for putting the evidence behind CBWC into practice at one NZ DHB ...26

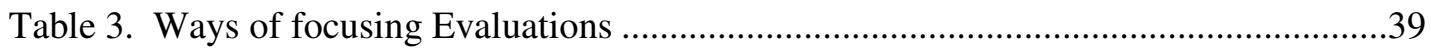

Table 4. The implementation and intervention timeline ......................................................62

Table 5. Number of births included in the audit ..................................................................

Table 6. Number of times the CBWC was calculated and recorded....................................78

Table 7. Comparison of recorded CBWC and CBWC calculated by myself .......................81

Table 8. Numbers of SGA and LGA babies identified by CBWC vs cut-off weights..........82

Table 9. 22 babies identified as SGA using CBWC who would not have been previously

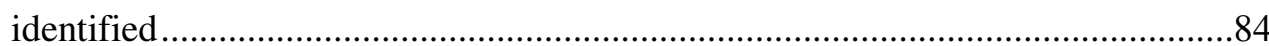

Table 10. 8 babies identified as LGA using CBWC who would not have been previously identified.......

Table 11. Babies recorded as SGA and whether BSL were initiated according to the guideline

Table 12. Babies recorded as LGA and whether BSL were initiated according to the guideline

Table 13. Ethnicity and birthweight of SGA and LGA babies identified by CBWC 


\section{List of Figures}

Figure 1. The classification of SGA babies by CBWC vs sex-adjusted population birthweight centiles.

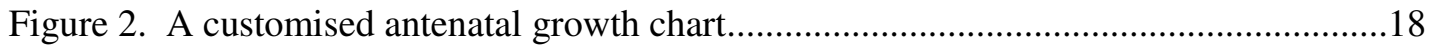

Figure 3. A customised birthweight centile calculator........................................................19

Figure 4. The knowledge-to-action framework ...............................................................29

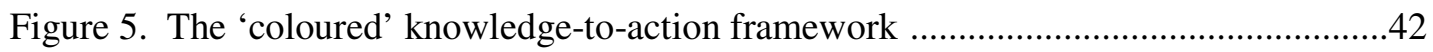

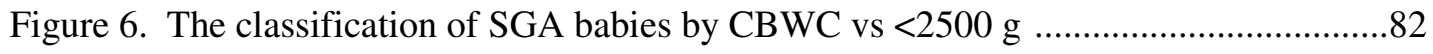

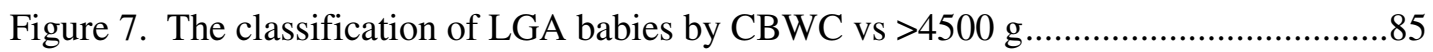

Figure 8. Simplified action cycle from the KTA framework ............................................104 


\section{Chapter One: Introduction}

When a baby is born, its birthweight is measured and an assessment is made to determine whether this weight is within a 'normal' range for the gestation. One reason why this is important is that babies who are either small or large for their gestation may be at risk of hypoglycaemia (Holtrop, 1993). Consequently, the method used to define small for gestational age (SGA) and large for gestational age (LGA) is crucial. It sets the threshold by which a newborn's blood sugar levels (BSL) will be monitored. Traditionally, cut-off points of $<2500 \mathrm{~g}$ and $>4500 \mathrm{~g}$ were used to identify SGA and LGA babies. But this only considers birthweight. Birthweight centiles are another tool commonly used. They consider gestation in addition to birthweight and are usually sex-adjusted. But often birthweight centile charts developed from a given population are then inappropriately applied to populations with different characteristics. Alternatively, customised birthweight centiles (CBWC) take into account birthweight as well as a number of variables of the pregnancy to give an 'individualised' or 'customised' centile for that baby. These variables are the mother's ethnicity, parity, height, booking weight and the baby's gender and gestation (McCowan, et al., 2004). The evidence shows that using a 'customised' centile will more accurately identify babies inappropriately grown for their gestation who are at increased risk of adverse perinatal events (De Jong, Gardosi, Dekker, Colenbrander, \& Van Geijn, 1998). This should be considered in assessing a baby's risk of neonatal hypoglycaemia.

To explain this further it is helpful to consider this scenario. A mother having her first baby gives birth to a $3 \mathrm{~kg}(6.6 \mathrm{lb})$ baby boy on her due date. Would you consider her baby to be an appropriate size? What if you also knew the mother was European, $157 \mathrm{~cm}$ (5ft 2") and had a booking weight of $50 \mathrm{~kg}$ ? Or what if the mother was Tongan, $170 \mathrm{~cm}$ (5ft 7") and had a booking weight of $120 \mathrm{~kg}$ ? Would your opinion about the size of the baby change depending on who the mother was? Taking all these variables (maternal ethnicity, parity, height, booking weight and 
baby gender and gestation) into consideration the European baby would have a CBWC of 19. The Tongan baby would have a CBWC of 2. If SGA is defined by a CBWC being less than 10, then only the Tongan baby would be identified as SGA. The Tongan baby would be considered at risk of hypoglycaemia and would have its BSL monitored. The European baby would be identified as appropriately grown for its gestation. It would not be considered at risk of hypoglycaemia and would not have its BSL monitored.

The concept of using customisation to accurately identify growth restricted babies is a relevant and timely issue, as demonstrated by the most recent Perinatal and Maternal Mortality Review Committee (PMMRC) report (PMMRC, 2011). Using CBWC they found "SGA was evident in 41 percent of perinatal related deaths at 24 weeks or beyond ... 42 percent of stillborn babies and 26 percent of neonates whose deaths were not due to congenital abnormality. This [was] significantly more frequent than the expected rate of 10 percent" (PMMRC, 2011, p. 55). As a result the PMMRC have recommended the use of customised growth charts in pregnancy (PMMRC, 2011). CBWC which are calculated at birth have not yet been nationally recommended and their application in NZ remains limited.

This thesis explores the use of CBWC in clinical practice. It looks at why and how CBWC were implemented at one NZ DHB. It also examines the results of implementing this change in practice. In wanting to describe what happened in a meaningful way, a knowledge translation framework was chosen to provide the theoretical foundation needed to interpret and analyse the processes that occurred. The study was a mixed methods process-outcome evaluation, overlayed with the knowledge-to-action (KTA) framework to explain the processes of implementation. After utilising the framework, it was decided that comment could also be made about the usefulness of the framework when applied to a clinical practice issue retrospectively. This resulted in a double layered thesis. In the forefront the focus is on the issue of using CBWC in clinical practice. In the background the usefulness of 
a knowledge translation framework is also highlighted. This thought process led to the development of the following Research Question and Objectives:

\section{Research Question}

How was the evidence behind the use of customised birthweight centiles (CBWC) put into practice at one New Zealand District Health Board (NZ DHB) and what are the outcomes resulting from translating this knowledge into action?

\section{Objectives:}

- To identify and critique the evidence relating to the use of CBWC

- To describe how CBWC were implemented at one NZ DHB

- To evaluate the processes followed when utilising CBWC

- To identify outcomes relating to babies identified as SGA or LGA using CBWC

- To evaluate the usefulness of the knowledge-to-action framework when applied to a clinical practice issue

The remainder of this introductory chapter will provide the background to how 'normal' birthweight has been defined and why this is important in relation to assessing risk of neonatal hypoglycaemia. The relevance of these issues will be explored by examining a Health and Disability Commissioner (HDC) report in which the Commissioner asked how practitioners can better identify babies at risk of hypoglycaemia based on their birthweight.

\section{Defining 'normal' birthweight}

The history of defining 'normal' birthweight has been inadequate and haphazard at best. In 1948 the First World Health Assembly accepted the definition for prematurity as a birthweight $\leq 2500 \mathrm{~g}$ and this was adopted almost universally to identify infants requiring special care (World Health Organization, 1961). In the 
1950 's an expert group recognised this definition wasn't applicable in some countries and that birthweight needed to be correlated with gestation (World Health Organization, 1961). It was recognised this standard overwhelmed the resources of some hospitals, so the birthweight was lowered to locally adapt the definition (World Health Organization, 1961). This meant there was no longer a uniform definition for babies requiring special care based on their birthweight.

Another expert committee found that "there is in reality no sharp dividing line between mature and premature babies or between high and low birth weight babies" (World Health Organization, 1961, p. 6). But due to the lack of basic data they were not able to clarify this definition further. Instead they recommended the definition of $\leq 2500 \mathrm{~g}$ be changed from defining pre-maturity to that of low birth weight (World Health Organization, 1961). Gestation was not included as part of this classification. This definition of $\leq 2500 \mathrm{~g}$, then became commonly used to identify SGA babies, with LGA babies commonly identified as those $>4500 \mathrm{~g}$ (Health and Disability Commissioner, 2007). Defining 'normal' birthweight is important because babies who are inappropriately grown for their gestation may be at increased risk of hypoglycaemia at birth. The way 'normal' birthweight is defined provides one criteria for which babies will be monitored for hypoglycaemia in the immediate newborn period.

\section{Hypoglycaemia in the newborn}

Hypoglycaemia occurs when low blood sugar levels (BSL) are present. In the newborn, undetected hypoglycaemia can lead to irreversible brain damage. "In newborns at risk, hypoglycaemia is most likely to occur in the first 24 hours of life, as the infant adapts to extrauterine life" (World Health Organization, 1997, p. 1). Glucose levels are the lowest 1-2 hours after birth. But a healthy term infant has a normal physiological response called counterregulation (World Health Organization, 1997), which can increase BSL above $2.5 \mathrm{mmol} / \mathrm{L}$ (Rozance \& Hay, 2006). A BSL concentration maintained around $2.5 \mathrm{mmol} / \mathrm{L}$ is generally accepted as a 'safe' level 
(Wight, Marinelli, \& The Academy of Breastfeeding Medicine Protocol Committee, 2006). But selecting an 'unsafe' level below which neurological damage will occur, remains highly debatable.

A consistent definition of hypoglycaemia does not exist in the literature (Rozance \& Hay, 2006). Cornblath et al., (2000) state that "the definition of clinically significant hypoglycaemia remains one of the most confused and contentious issues in contemporary neonatology" (p. 1141). In addition there is no definitive time frame known in which neurological injury can occur (Rozance \& Hay, 2006). This is because deciding whether a BSL is 'safe' or not is influenced by birthweight, gestation, postnatal age and feeding method. So any definition would likely be too rigid to apply universally (World Health Organization, 1997). Therefore "all that can be proposed are pragmatic intervention thresholds that also provide a margin of safety" (Cornblath, et al., 2000, p. 1143). Hypoglycaemia may indicate that malnutrition in-utero has occurred (Gardosi, Mongelli, \& Mul, 1995). Except for babies born to mothers with poorly controlled diabetes, hypoglycaemia is more common in growth restricted babies than any other group (Halliday, 2009).

Some SGA babies are unable to produce an adequate metabolic and endocrine response to hypoglycaemia (Cornblath, et al., 2000). SGA babies may be at increased risk of hypoglycaemia due to a high brain to body mass ratio, reduced fat stores and hyperinsulinism (World Health Organization, 1997). A baby identified as SGA will have its BSL closely monitored in an attempt to prevent some degree of morbidity or possible mortality (Goldenberg \& Cliver, 1997). But there is a lack of research on how to more precisely identify SGA babies and the incidence and outcomes of hypoglycaemia in these babies (World Health Organization, 1997). The risks of hypoglycaemia for LGA babies are discussed to a lesser degree in the literature.

Metabolic studies have not been as extensively studied in LGA infants (World Health Organization, 1997), but macrosomia may be associated with a higher risk of 
neonatal hypoglycaemia (Wollschlaeger, Nieder, Koppe, \& Hartlein, 1999). A baby who is physiologically LGA has proportionate increases in brain size and abdominal circumference. Whereas a LGA baby born to a diabetic mother will have increased fat, muscle and liver mass due to the effects of increased insulin (World Health Organization, 1997). LGA babies have previously been considered 'at risk' as it was believed they represented babies born to mothers with undiagnosed diabetes (Wight, et al., 2006). But this view remains controversial. Some have suggested that all LGA babies of non diabetic mothers should be monitored for hypoglycaemia, but for a shorter period (Holtrop, 1993). While others believe routine glucose monitoring should be performed on all LGA babies of non-diabetic mothers (Schaefer-Graf et al., 2002). More recently the literature favours LGA babies not being considered 'at risk' when maternal diabetes is absent (UNICEF, 2008). This means screening women for diabetes in pregnancy can eliminate LGA as a risk factor for those babies which are physiologically versus pathologically large.

\section{The Health and Disability Case (Case 05HDC16723)}

Birthweight as a risk factor for neonatal hypoglycaemia was highlighted by a recent HDC case. The report was a timely reminder for DHBs around the importance of identifying babies at risk and recognising, then rectifying, hypoglycaemia. In this case (Health and Disability Commissioner, 2007) a $2735 \mathrm{~g}$ male baby was born at $38+5$ weeks gestation by caesarean section for foetal distress in early labour. The mother smoked in pregnancy and was taking the anti-depressant Aropax. Although the baby breastfed well initially, by Day Three the baby was reluctant to feed and became hypoglycaemic. Despite intervention at this point the baby was unable to maintain adequate BSL. Due to the period of hypoglycaemia, the baby suffered severe neurological problems. Expert midwifery opinion for the case confirmed the baby's birthweight of $2735 \mathrm{~g}$ would have been considered normal, as <2500 g was the cut-off for SGA and low birthweight babies in many hospitals. 
"Weight of 2500 gms would have been used as a cut off point in 2004 rather than customised growth charts to assess individual growth. Customised growth charts are not a normal assessment tool in all hospital/units within NZ even in 2007. He therefore was not considered to be at risk of hypoglycaemia" (Health and Disability Commissioner, 2007, p. 20).

Although the term customised growth charts (GCG) was used by the expert midwife, in relation to identifying the normality of a baby's birthweight, a customised birthweight centile (CBWC) would provide a more accurate guide of appropriate growth at birth for a given gestation, rather than the CGC. CGC are used antenatally, whereas CBWC are calculated at birth. A CGC therefore estimates foetal growth in pregnancy based on serial plotted fundal height measurements. But a CBWC gives a 'customised' birthweight centile based on the actual birthweight. But both CGC and CBWC were developed from the same software. Throughout the HDC report CGC is referred to when mentioning the baby's birthweight of $2735 \mathrm{~g}$. But to provide the correct context for the comments made in the report, it would have been more accurate for CBWC to be referred to in relation to considering whether the baby's birthweight was 'normal'. In his final opinion the Commissioner states, "I am advised that the customised growth charts [CBWC], which are a more reliable guide for staff [in identifying birthweight as a risk factor], are even now not in common usage throughout New Zealand" (Health and Disability Commissioner, 2007, p. 31). The HDC findings were sent to all DHB CEO's and other national organisations for educational purposes. This case highlighted the need for DHBs to review their neonatal hypoglycaemic management policies and how they identified babies 'at risk'. It also proposed using CBWC to assess individual birthweight as part of that risk assessment.

\section{Better identifying babies at risk of hypoglycaemia due to their birthweight}

A study in the 1970's found that episodes of hypoglycaemia were being missed in some babies who were $>2500 \mathrm{~g}$. These babies had likely experienced intra-uterine 
malnutrition and their actual birthweight fell short of its genetic potential (Oakley \& Parsons, 1977). They determined that more research needed to be completed to see whether it was possible to predict which infants $>2500 \mathrm{~g}$ would suffer from neonatal hypoglycaemia and to determine an objective measure of malnourishment in babies $>2500$ g (Oakley \& Parsons, 1977). Since then others have also realised that assessment of gestational age and appropriateness of size for gestation should be a part of determining this risk (Rozance \& Hay, 2006). Perhaps CBWC, which considers the optimum growth potential of a baby by taking a number of variables of the pregnancy into account, could be the 'objective measure' referred to.

\section{My person practice perspective}

My midwifery career began in 2005 and I have worked as a caseload midwife in the community and a hospital-based midwife within secondary and tertiary settings. During this time I also became a mother and commenced my postgraduate studies. I am a Maori midwife who developed a love of learning from my parents and I view ongoing education as an important part of my personal and professional development. I had always wanted to complete a piece of research and publish the findings in a journal. While working as a midwife I looked for an interesting topic to research relating to clinical practice. As a student and then a qualified midwife, I observed that people were often resistant to changing the way they practiced. Often midwives reasoned they had always done it that way and saw no reason to change. I also noticed that DHBs had different ways of practicing even though each guideline claimed to be 'evidenced-based'. One policy difference I noticed was how SGA and LGA babies were identified in relation to their risk of hypoglycaemia. I work at a DHB who use cut-off birthweights at varying gestations adapted from population centile charts. I later became aware of another DHB who were using CBWC. I wondered what the rationale was for using either method. I also wondered which method was more successful at identifying babies at risk of hypoglycaemia based on birthweight. 
I chose CBWC as the topic for my thesis because using customisation to identify growth restricted babies is a contemporary issue. The majority of health practitioners I've spoken with have never heard of CBWC. So it appeared the DHB, that was using CBWC was innovative and pioneering in their use of CBWC. I wondered why this DHB chose to use CBWC and what difference it had made in the outcomes for babies. There didn't appear to be any studies describing the practical issues around implementing CBWC. There were no studies which investigated the use of CBWC to identify risk of hypoglycaemia in the newborn. So I believed the findings of my research could potentially be of significant interest to a number of health professionals.

\section{Structure of the thesis}

This thesis tells the story of how one NZ DHB implemented CBWC at their maternity unit. The thesis is divided into six chapters. This introductory chapter has provided the background of how using birthweight to identify risk of hypoglycaemia is relevant and how a recent HDC case highlighted this issue. Chapter Two discusses the evidence that exists for using customised standards, which led to the development of CBWC. Chapter Three will discuss the concept of knowledge translation and introduce the knowledge-to-action theoretical framework used as a research tool. Chapter Four will provide the justification for selecting a mixed methods evaluation approach and describes the overall research design and methods. The results of the research will be presented in Chapter Five. Chapter Six will elaborate on some of the more significant findings, including discussing implications, recommendations and ideas for further research. The thesis ends with an overall conclusion and numerous appendices have been attached which are referred to throughout the thesis. 


\section{Summary}

The history of how 'normal' birthweight and hypoglycaemia have been defined is haphazard and controversial. Each definition depends on a number of variables and no single definition is applicable to all clinical scenarios. The importance of recognising birthweight as a risk factor for hypoglycaemia was highlighted by a HDC case where a baby developed irreversible brain damage. The Commissioner noted that using CGC was a more reliable guide for staff, but in the context of the report in relating to identifying risk based on birthweight, what should have been discussed was the use of CBWC as a more reliable guide for staff. The use of CBWC is still not common practice within NZ DHBs. This topical issue interested me as I was aware of one NZ DHB who calculated a CBWC after every birth. I wanted to investigate why and how they implemented CBWC and the outcomes resulting from this change. It is hoped this thesis will cause individuals and DHBs to consider the value of $\mathrm{CBWC}$ as a clinical tool in assessing the normality of birthweight and its relationship with the risk of neonatal hypoglycaemia. In the next section I will explain in detail what CBWC are and how they were developed. The evidence for using 'customised' standards will also be provided. 


\section{Chapter Two: Customised Birthweight Centiles}

\section{Introduction}

Over the last 50 years the importance of considering gestation when defining 'normal' birthweight has been recognised (World Health Organization, 1961). Additionally, a number of physiological and pathological maternal and foetal variables are known to affect birthweight (Gardosi, Mongelli, Wilcox, \& Chang, 1995). This chapter introduces some of the terminology used to define appropriate birthweight and how it relates to the way 'small' and 'large' babies are identified. It discusses how the GROW (Gestation Related Optimum Weight) software was developed. This was then used to create customised growth charts (CGC) which monitor foetal growth in pregnancy and customised birthweight centiles (CBWC) which are calculated at birth. The findings from the main studies on customising standards are presented. The majority of the studies conclude that using CBWC as a classification standard results in more accurately identifying those babies who are truly growth-restricted, or pathologically large. This represents babies that are at increased risk of perinatal morbidity and mortality. Finally a timeline is also presented to show how long it took for this evidence to be implemented at the DHB in this research study.

\section{Understanding the terminology}

The terms IUGR (intrauterine growth restriction), SGA (small for gestational age) and LBW (low birth weight) are often used interchangeably. SGA babies are also sometimes known as small for dates (SFD). While it is possible that a baby might be all of these, this may not necessarily be true. IUGR is a "reduced growth in relation to the fetus' genetic potential and small for date infants may be the result of individual normal genetic variation rather than poor growth" (Ego et al., 2006, p. 1042). IUGR babies are labelled according to an antenatal assessment of their growth, whereas SGA babies are labelled according to an assessment of their 
birthweight (Maulik, 2006). A LBW baby is born at term ( $\geq 37$ weeks) weighing $<2500$ g (World Health Organization, 1961). LGA (large for gestational age) babies are also labelled according to an assessment of their birthweight. Definitions of macrosomic (large) babies range from $>4,000 \mathrm{~g}$ to $>5000 \mathrm{~g}$ regardless of gestation, with $\geq 4500$ g most commonly used (Heiskanen, Raatikainen, \& Heinonen, 2006). Comprehending the subtle differences between some of these definitions is important in understanding how these terms relate to $\mathrm{CGC}$ and $\mathrm{CBWC}$. For example, CGC identifies foetal growth restriction in pregnancy (IUGR) and CBWC identifies foetal growth restriction at birth (SGA). It is important that the correct terminology is used and understood in the appropriate context relating to how 'small' and 'large' babies are identified.

\section{Accurately identifying SGA and LGA babies}

SGA babies have an increased risk of perinatal morbidity and mortality and at the other extreme LGA babies have a similar increased risk of morbidity (Beeby, Bhutap, \& Taylor, 1996). Choosing the most accurate method to identify these babies at birth is important as this will determine which ones require additional care. Some methods, to determine which babies require additional care, but not considering gestation have been developed such as: weight to length ratios, ratios of anthropometric measurements, neonatal skinfold thickness, body fat and body composition (Goldenberg \& Cliver, 1997). But most traditionally a baby $<2500$ g or $>4500 \mathrm{~g}$ has been considered SGA or LGA. Population centile charts are sometimes used which take gestation into consideration and are usually sex-adjusted. Population centile chart cut-off points have been arbitrarily chosen to define SGA at the $10^{\text {th }}, 5^{\text {th }}$ or $3^{\text {rd }}$ centile or two standard deviations below the mean (Gardosi, 2009a). A cut-off below the $10^{\text {th }}$ centile for a given gestation is the most common definition applied for SGA babies (Maulik, 2006). The most common cut-off for LGA babies is above the $90^{\text {th }}$ centile (Gardosi, Chang, Kalyan, Sahota, \& Symonds, 1992). But the reliability of these classification methods have been questioned when applied to populations with varying characteristics. It has been proposed that 
CBWC are more appropriate to use (Zhang, Platt, Cnattingius, Joseph, \& Kramer, 2007). CBWC take several maternal and foetal characteristics relating to the pregnancy into consideration which 'customises' a birthweight centile for each individual baby. A cut-off centile still needs to be selected to identify a baby as SGA or LGA. But the centile will be 'customised' for that baby, instead of being based on conventional population standards.

\section{CBWC as a classification standard}

CBWC appear to be a more accurate measure because they consider the baby's potential for growth, rather than using arbitrary cut-off points based on population standards.

A customised birthweight centile reflects a greater range of physiologic characteristics of the pregnancy namely: booking weight, height, parity, ethnicity and infant gestation at delivery and sex. Using these data the centile calculator generates a customised birthweight centile for that individual baby. (McCowan \& Stewart, 2004, p. 435)

Using unadjusted population centiles will lead to a number of at risk babies being missed and a number of babies being incorrectly identified (Gardosi, Clausson, \& Francis, 2009). In their original study, Gardosi et al., (1992), found that when physiological variables are not taken into account around a quarter of babies identified as SGA or LGA were false-positives. These babies would have been physiologically rather than pathologically small or large. They also found that around a quarter of babies who should be SGA or LGA weren't identified when using conventional centiles.

This is demonstrated in Figure 1 below, which represents all SGA babies in a given population and how they would be identified if CBWC were used as a classification method versus sex-adjusted population centiles. One subset of the population will be identified as SGA by CBWC only; one subset will be identified as SGA by both 
methods and another subset will be identified as SGA by sex-adjusted population centiles only. The SGA by CBWC only subset will consist of babies who are pathologically small and who otherwise would have been missed. These babies would have been considered as having a normal birthweight on a population based centile chart, but are actually growth restricted (Cowan, 2009). The SGA by sexadjusted population centiles only subset will consist of babies who would have been considered false-positives. If based on CBWC they would not have been identified as growth restricted. A similar figure could be drawn to represent all LGA babies in a given population. (Note: In Figure 1 the size of the circles and overlap is not drawn to scale).

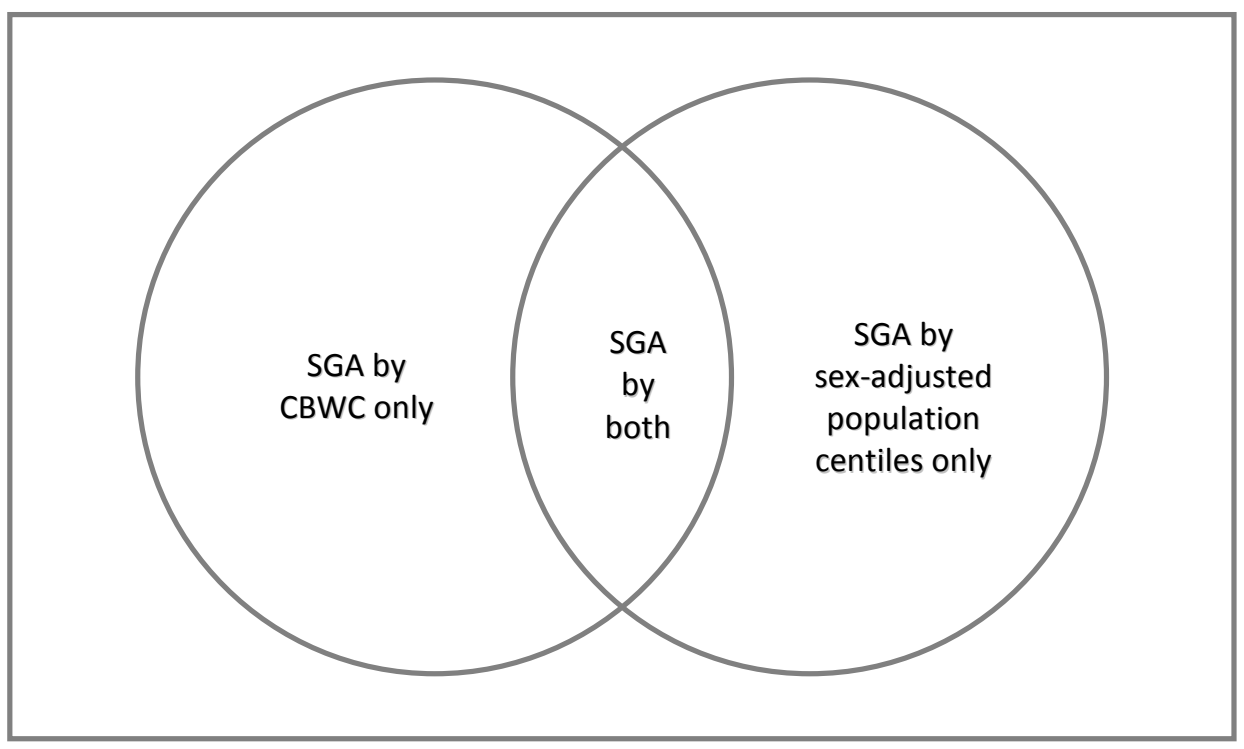

Figure 1. The classification of SGA babies by CBWC vs sex-adjusted population birthweight centiles

CBWC are a more reliable classification method as it defines 'normal' birthweight by the optimal growth potential of each individual baby (Gardosi, 2006). Using CBWC can reduce unnecessary intervention and decrease parental anxiety by identifying babies that are truly pathologically small or large for their gestation (Gardosi, et al., 1992). CBWC predicts an optimal birthweight based on a number 
of maternal and foetal variables. The software programme GROW was used to develop CBWC which considered these variables.

\section{The development of 'GROW' (Gestation Related Optimum Weight)}

The development of the GROW software by Gardosi et al., (1992) built upon previous concepts of individualised growth charts, but included factors that influenced the growth potential of the baby in-utero. It has been said that "birthweight is a biological variable with corresponding variability" (Bakketeig \& Magnus, 1992, p. 140), meaning that birthweight is influenced by a number of factors. Factors such as smoking, low socio-economic status, pre-eclampsia and diabetes were found to have a pathological influence on birthweight. So they were not included in the development of GROW as they were trying to predict a baby's growth within an optimum environment (Gardosi, 2009b). The variables: gestation, foetal sex, booking weight, height, ethnicity and parity were shown to be independent determinants of birthweight and were included in the model (Gardosi et al., 1992). GROW determined the level of influence these variables had on birthweight.

\section{Gestation and Parity}

As early as the 1960's it was understood that gestation influenced whether a birthweight should be considered 'normal' (World Health Organization, 1961). It was identified that infants of similar birthweight, but different gestational age, developed dissimilar clinical problems (Battaglia \& Lubchenco, 1967). Various classification systems based on birthweight and gestation were therefore proposed. Battaglia and Lubchenco (1967) divided gestational age into three divisions referred to as Pre-Term, Term and Post-Term and this determined which type of nursery care the baby received. This made it easier to identify which babies were SGA and LGA. Other birthweight centile charts were developed which took into account whether previous births were SGA or LGA. This reflected "the tendency to repeat weight for 
gestation in successive births" (Bakketeig \& Magnus, 1992, p. 142). It is known that gestation has the strongest influence on birthweight of about $20 \mathrm{~g}$ per day at term (Gardosi, Mongelli, \& Mul, 1995). So accurately dating pregnancies and calculating gestation is important. Parity is also a statistically significant determinant of birthweight (Voorhorst, Bouter, Bezemer, \& Kurver, 1993). And it has been shown that infants of nulliparous women weigh less than infants of multiparous women (Goldenberg \& Cliver, 1997).

\section{Sex and Ethnicity}

Male infants on average weigh more than female infants (Goldenberg \& Cliver, 1997). In developing NZ birthweight centiles one study found that at each gestation week, male babies were approximately 100g heavier than female babies (Thompson, Mitchell, \& Borman, 1994). McCowan and Stewart (2004) also found that there were significant differences in birthweights across NZ ethnic groups. Tongan and Samoan babies were found to be significantly heavier than all other ethnic groups. The understanding that ethnicity has a significant effect on birthweight has long been recognised (Grundy, Hood, \& Newman, 1978). Obvious differences between ethnic groups have been found as various birthweight databases were developed (Gardosi, Mongelli, Wilcox, et al., 1995). This variation in birthweight across ethnicities supports the argument that "continued use of birthweight standards derived from predominantly European or Caucasian populations for all racial/ethnic groups is clearly undesirable" (Gardosi, et al., 1992, p. 286). It has also been suggested that these differences appear to be mainly the result of characteristics such as maternal height and weight (Voorhorst, et al., 1993).

\section{Maternal Height and Weight}

In the 1960's it was recognised that birthweight was influenced by maternal height and weight. After gestation, maternal weight at booking was shown to have the strongest correlation with birthweight (World Health Organization, 1961). This correlation extends further in that there is a significant difference between the average height and weight for mothers of SGA and non-SGA babies. Not surprisingly, smaller mothers are more likely to have smaller babies (De Jong, et al., 
1998). Alternatively, Cnattingius, Bergstrom, Lipworth and Kramer (1998), found that a higher maternal weight protects against having a SGA baby. This research was based on population rather than customised standards and this finding was disputed by Gardosi, Clausson and Francis (2007). They found that using population standards underestimates the contribution of obesity to SGA risk. They also found that a high maternal body mass index (BMI) is associated with an increased number of SGA babies when customised centiles are used (Gardosi \& Francis, 2009a). A high BMI is an important risk factor which should be identified early in pregnancy (McCowan, et al., 2004). Increases in maternal BMI are directly proportional to perinatal mortality (Gardosi, et al., 2009). This is one risk factor of pregnancy which is preventable (Cnattingius, et al., 1998). Smoking in pregnancy is another preventable risk factor, which women have the ability to influence.

\section{Smoking}

Smoking has a significant and independent growth restricting effect (Gardosi, Mongelli, Wilcox, et al., 1995) and has a "dose-response relationship" with birthweight (Gardosi, 2009a, p. 742). Because this effect is pathological, expectant birthweight should be calculated as if the mother was a non-smoker (Gardosi, Mongelli, Wilcox, et al., 1995). The GROW software takes this into account and "the resultant growth curve ... represents an ideal standard for each individual pregnancy, which would be more sensitive for identifying any growth retardation due to smoking" (Gardosi, Mongelli, Wilcox, et al., 1995, p. 173). A mother who smokes does not provide the optimal environment in which their baby is expected to grow and this has a significant effect on birthweight.

It was already known that birthweight standards needed to be developed for the specific population they are applied to (Sloan \& Lorenz, 1991). So "to optimise antenatal growth screening and assessment, the physiological variables that are known to affect birthweight also need to be applied to fetal weight curves" (Gardosi, Mongelli, Wilcox, et al., 1995, p. 173). The GROW software achieved this by taking physiological variables affecting birthweight into consideration and was then used to create CGC and CBWC. 


\section{Customised Growth Charts (CGC)}

Gardosi et al., (1995) set out to make a model that could provide an adjustable foetal weight standard, by which a predicted ideal birthweight could be measured against the observed birthweight. This meant the median and centile values of a growth chart were not fixed, as the values could be affected by different characteristics. They were able to combine these maternal characteristics and previous birthweights with a "standard, longitudinal ultrasound-derived curve for intrauterine weight gain" to create a "computer-generated antenatal chart that can be easily "customised" for each individual pregnancy" (Gardosi, et al., 1992, p. 283). The software programme can be downloaded for free from www.gestation.net for UK, Australian, NZ and USA populations, with other international versions being developed. Maternal variables entered into the programme at the first antenatal visit will generate a chart (Figure 2), which can be used to monitor foetal growth throughout the pregnancy.

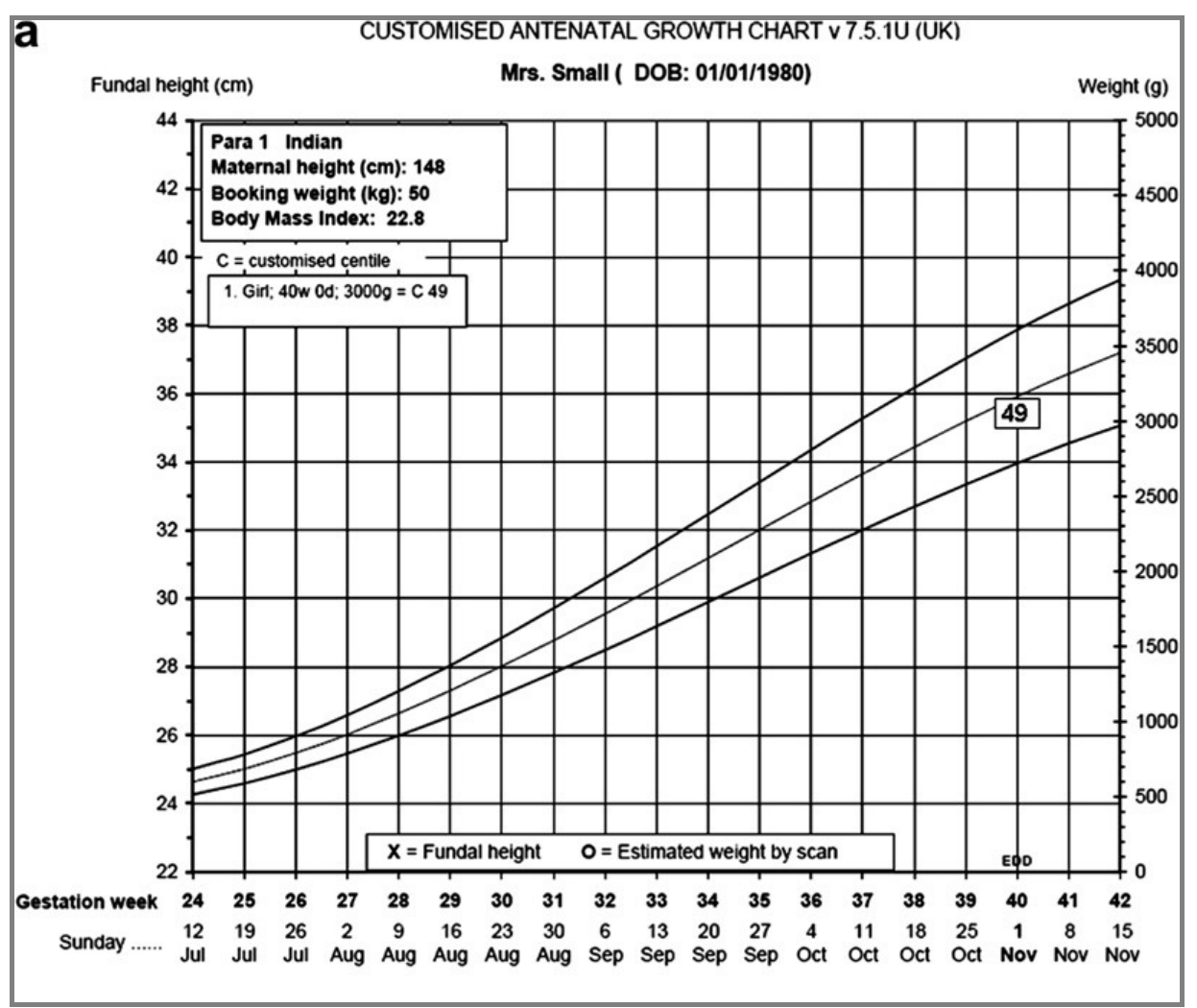

Figure 2. A customised antenatal growth chart (Gardosi, 2009a, p. 743) 
The use of CGC has been recommended by the PMMRC for all pregnancies in NZ to improve the recognition of foetal growth restriction antenatally (PMMRC, 2011). While fundal heights are plotted on a CGC throughout pregnancy, a CBWC is calculated in the postnatal period once the actual birthweight is known.

\section{Customised Birthweight Centiles (CBWC)}

The CBWC calculator can also be downloaded for free from www.gestation.net (Figure 3). The calculator is used by selecting a value from each of the drop down menus corresponding to each variable and a CBWC is automatically calculated. Figure 3 demonstrates the data values from the birth of my first child, giving a CBWC of 19.

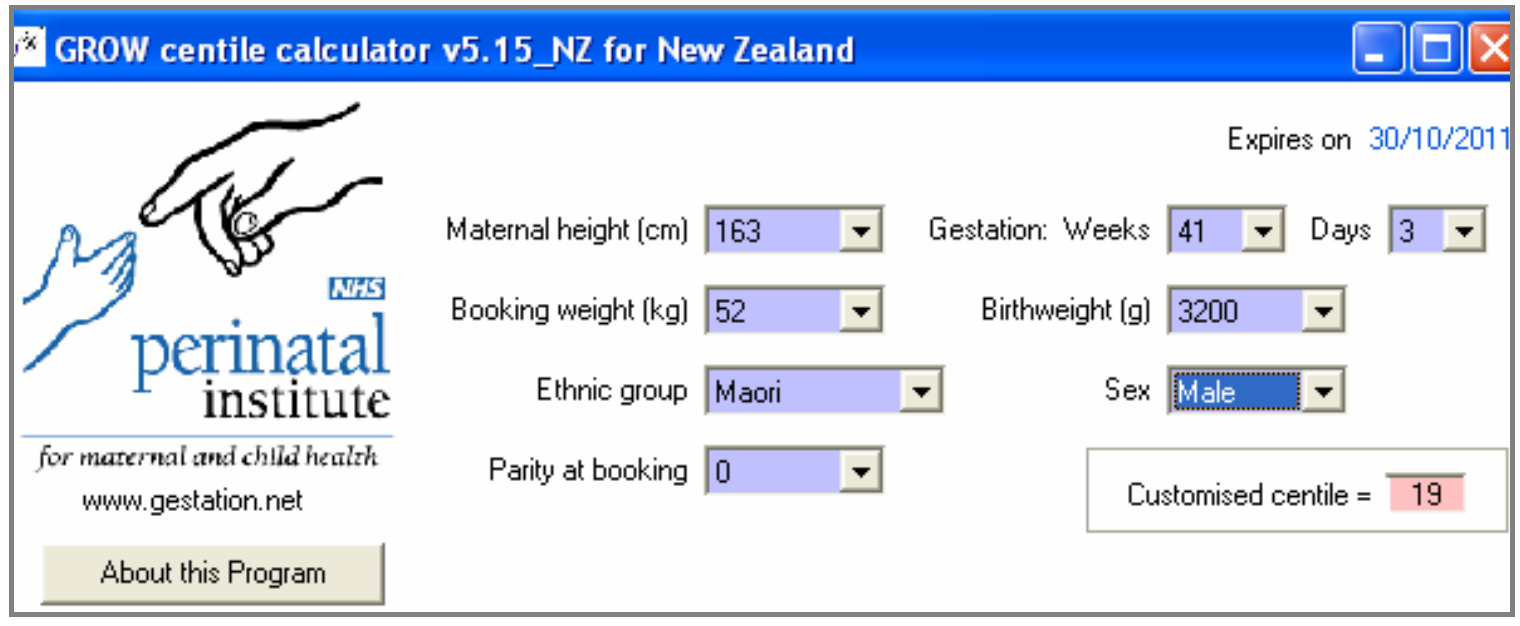

Figure 3. A customised birthweight centile calculator (www.gestation.net)

Adjusting any of the data values will accordingly change the CBWC result. In this example changing ethnicity to Tongan gives a CBWC of 10. Changing gestation to 39 weeks increases the $\mathrm{CBWC}$ to 53 . Increasing the booking weight to $90 \mathrm{~kg}$ lowers the CBWC to 7. A 'normal' $\mathrm{CBWC}$ is determined by the cut-off centile level and will likely vary across maternity providers. A cut-off below the $10^{\text {th }}$ centile is the most common definition applied for SGA (Maulik, 2006) and a cut-off above the $90^{\text {th }}$ centile for LGA babies (Gardosi, et al., 1992). The DHB in the study defined a 
SGA baby as having a CBWC less than the $10^{\text {th }}$ centile and a LGA baby as having a CBWC greater than the $95^{\text {th }}$ centile. Therefore, any CBWC value in the range of 10 to 95 was considered normal at the DHB.

Using CBWC has been recommended by the British Royal College of Obstetricians and Gynaecologists (Hutcheon, Zhang, Cnattingius, Kramer, \& Platt, 2008). It has also been suggested that American obstetricians adopt the use of customised foetal growth standards in customising birthweight (Resnik, 2007). The PMMRC calculates a CBWC for all foetal deaths in NZ when analysing perinatal mortality data and define SGA as a CBWC less than the $10^{\text {th }}$ centile (PMMRC, 2011). But their application has not yet been recommended nationally, even though a CBWC calculator for $\mathrm{NZ}$ is currently available.

\section{Development of CBWC for a NZ population}

McCowan and Stewart (2004) determined that differences in birthweight across major ethnic groups in NZ were significant. They developed NZ ethnic specific birthweight centile charts (for gestations 38 to 41 weeks) for both sexes. The 10th and 90th percentile was calculated for ethnicities and the cut-off points were found to be different (McCowan \& Stewart, 2004). This was the first study to report birthweight centiles for common ethnic groups in NZ. Part of this data was applied to the GROW software and used to develop CGC and a CBWC calculator for a NZ population. When applied to the model, half of the data had to be disregarded because maternal weight and/or height weren't available. They concluded that maternal height and weight at booking had to be routinely collected before customised centiles could be fully applied in a NZ setting (McCowan, et al., 2004). While the CBWC calculator is readily available, many practitioners are unaware of CBWC and the numerous studies advocating for the 'customising' of standards. 


\section{The evidence for and against 'customising' standards}

The GROW software was used to develop both CGC and CBWC. Therefore an argument supporting or opposing 'customising' standards in the antenatal period would also be applicable to the postnatal period. The main studies from a range of countries which provides the evidence for and against using 'customised' standards verses population based standards are outlined in Table 1 below. These studies are all examples of retrospective reviews of population databases. There are no randomised control trials. The majority of these studies were about customisation being used to identify SGA babies. Only one study specifically mentioned LGA babies. Most studies advocated the use of customised standards as the most accurate method. A few studies provided arguments against the use of customisation, but their findings have since been critiqued by the original authors of the research which was being challenged.

Studies advocating the use of customisation found that babies identified as SGA using customised standards had the highest rates of perinatal morbidity and mortality (De Jong, et al., 1998; Gardosi \& Francis, 2009a). This included a higher risk of stillbirth and neonatal death (Clausson, Gardosi, Francis, \& Cnattingius, 2001; Ego, et al., 2006; Gardosi, et al., 2009). For other measures of perinatal morbidity and mortality, there were similar rates in the SGA by customisation only group and the SGA by both methods group (McCowan, Harding, \& Stewart, 2005). Babies identified as SGA using population centiles only, had levels of perinatal morbidity similar to a non-SGA population (Clausson, et al., 2001). This relates back to Figure 1 (p. 14) introduced earlier in this chapter which illustrated three different subsets of babies identified as SGA using CBWC versus sex-adjusted population centiles. The CBWC group uses 'customised' standards, versus the sex-adjusted population centile group which uses population standards. The common conclusion of the studies was that customised centiles are better at truly identifying babies 'at risk' of adverse perinatal outcomes due to their birthweight (Clausson, et al., 2001; Gardosi, et al., 2009). Therefore, customising standards improves the diagnosis of growth restriction (Groom, Poppe, North, \& McCowan, 2007). 
Studies arguing against customising standards were around issues relating to the customisation technique of Gardosi et al., (1992). Some believed the effect of several variables had been incorrectly estimated, which produced an exaggerated increase in perinatal risk (Zhang, et al., 2007). Zhang et al., (2007) said that the finding of an increase in the risk of perinatal mortality observed with customised centiles was 'largely an artefact' and due to a higher proportion of preterm babies identified as SGA. But Gardosi et al., (2007) explained that "gestational age patterns become apparent when the appropriate standard is used. They are of interest in the study of adverse outcomes in pregnancy and are hardly an artefact" (Gardosi, et al., 2007, p. 1301). McCowan et al., (2007) also argued that the ability of customised centiles to identify an increased proportion of SGA infants born preterm is in fact one of the important advantages of using customised centiles over population centiles. Hutcheon et al., (2008) argued that customising for maternal variables makes little difference. But Gardosi and Francis (2009a) argued Hutcheon et al., (2008) had only partially applied their model, which would have affected the results. Finally, Ego et al., (2008) found that excluding parity from customising SGA babies made no difference in identifying high risk babies (Ego, et al., 2008). Gardosi et al., (2009) performed their own analysis to measure the effect of perinatal mortality risk associated with parity. They commented that Ego et al., (2008), had failed to look at the whole parity spectrum and that when all parity groups are considered, customising SGA babies better reflects perinatal mortality risk (Gardosi \& Francis, 2009c). 
Table 1. Studies providing the evidence for and against using 'customised' standards verses population based standards

\begin{tabular}{|c|c|c|}
\hline Authors and Title & Type of study & Results and Conclusions \\
\hline \multicolumn{3}{|c|}{ Studies advocating using customised standards: } \\
\hline $\begin{array}{l}\text { (Gardosi, et al., 1992) } \\
\text { Customised antenatal } \\
\text { growth charts. }\end{array}$ & $\begin{array}{l}\text { Retrospective } \\
\text { review. } \\
4179 \text { pregnancies } \\
\text { in the UK. }\end{array}$ & $\begin{array}{l}\text { - Using adjusted centiles } 28 \% \text { of babies designated SGA and } 22 \% \text { of those designated LGA were } \\
\text { actually within normal limits for the pregnancy. } \\
\text { - } 24 \% \text { and } 25 \% \text { of babies identified as SGA or LGA with adjusted centiles were not identified by } \\
\text { conventional unadjusted centiles. } \\
\text { - Babies who were SGA by conventional unadjusted centiles only, had significantly fewer } \\
\text { instances of low Apgar scores. }\end{array}$ \\
\hline $\begin{array}{l}\text { (De Jong, et al., 1998) } \\
\text { Application of a } \\
\text { customised birthweight } \\
\text { standard in the assessment } \\
\text { of perinatal outcome in a } \\
\text { high risk population. }\end{array}$ & $\begin{array}{l}\text { Retrospective } \\
\text { comparison. } \\
217 \text { babies from } \\
\text { high risk } \\
\text { pregnancies in the } \\
\text { Netherlands. }\end{array}$ & $\begin{array}{l}\text { - Customising resulted in an increase of SGA babies from } 14 \% \text { to } 31 \% \text {. } \\
\text { - Customised centiles significantly improves the identification of infants who have failed to reach } \\
\text { the expected birthweight and are at increased risk for adverse perinatal outcomes eg C-section } \\
\text { for foetal distress, NICU admission, ventilation, apgar }<7 \text { at } 5 \mathrm{~min}, \mathrm{pHa} \leq 7.15 \text {, stillbirth etc }\end{array}$ \\
\hline $\begin{array}{l}\text { (Clausson, et al., 2001) } \\
\text { Perinatal outcome in SGA } \\
\text { births defined by } \\
\text { customised vs population- } \\
\text { based birthweight } \\
\text { standards. }\end{array}$ & $\begin{array}{l}\text { Population based } \\
\text { cohort study. } \\
326,377 \text { births in } \\
\text { Sweden. }\end{array}$ & $\begin{array}{l}\text { - Risk of stillbirth, neonatal death and apgar score }<4 \text { at } 5 \text { mins were higher if SGA was classified } \\
\text { by customised vs population based birthweight standards. } \\
\text { - Compared to population based centiles, customised standards are better at identifying } \\
\text { foetuses with growth restriction associated with an increased risk of adverse perinatal outcome. } \\
\text { - Babies SGA by population standards, but not by customised standards, have little or no } \\
\text { increase in perinatal mortality. }\end{array}$ \\
\hline $\begin{array}{l}\text { (McCowan, et al., 2005) } \\
\text { Customised birthweight } \\
\text { centiles predict SGA } \\
\text { pregnancies with perinatal } \\
\text { morbidity. }\end{array}$ & $\begin{array}{l}\text { Cohort study. } \\
374 \text { SGA } \\
\text { pregnancies and a } \\
\text { general obstetric } \\
\text { population of } \\
12,879 \text { births. }\end{array}$ & $\begin{array}{l}\text { - Abnormal Doppler studies, nursery admission, long hospital stay, pre-eclampsia in the mother } \\
\text { and C-section for foetal distress, were less common in the population SGA only group. } \\
\text { - No perinatal deaths occurred in the population SGA only group. } \\
\text { - There were similar rates of perinatal morbidity and mortality between the customised SGA } \\
\text { only group and SGA both (customised and population) group. } \\
\text { - Using customised centiles is more likely to detect more babies at risk of perinatal morbidity } \\
\text { and mortality than using population centiles. }\end{array}$ \\
\hline
\end{tabular}




\begin{tabular}{|c|c|c|}
\hline $\begin{array}{l}\text { (Ego, et al., 2006) } \\
\text { Customized versus } \\
\text { population-based birth } \\
\text { weight standards for } \\
\text { identifying growth } \\
\text { restricted infants: A French } \\
\text { multicenter study. }\end{array}$ & $\begin{array}{l}\text { Retrospective } \\
\text { comparison. } \\
56,606 \text { births in } \\
\text { France. }\end{array}$ & $\begin{array}{l}\text { - CBWC identified a group of infants ( } 3 \%) \text { that would otherwise be considered appropriate for } \\
\text { gestational age using population standards. These infants had an increased risk of stillbirth and } \\
\text { perinatal death compared to non SGA babies. } \\
\text { - Individual growth norms adjusted for maternal and neonatal characteristics to identify } \\
\text { foetuses at risk should be used to define SGA. }\end{array}$ \\
\hline $\begin{array}{l}\text { (Gardosi \& Francis, 2009a) } \\
\text { Adverse pregnancy } \\
\text { outcome and association } \\
\text { with small for gestational } \\
\text { age birthweight by } \\
\text { customized and } \\
\text { population-based } \\
\text { percentiles. }\end{array}$ & $\begin{array}{l}\text { Retrospective } \\
\text { analysis of } \\
\text { database. } \\
35,235 \text { births in } \\
\text { the USA. }\end{array}$ & $\begin{array}{l}\text { - Babies by customised SGA showed a higher risk for threatened preterm labour, antepartum } \\
\text { haemorrhage, pregnancy-induced hypertension, preeclampsia, stillbirth and early neonatal } \\
\text { death, compared with SGA by population standards. } \\
\text { - Customised SGA differentiates between physiologically and pathologically small babies. }\end{array}$ \\
\hline $\begin{array}{l}\text { (Gardosi, et al., 2009) } \\
\text { The value of customised } \\
\text { centiles in assessing } \\
\text { perinatal mortality risk } \\
\text { associated with parity and } \\
\text { maternal size. }\end{array}$ & $\begin{array}{l}\text { Population-based } \\
\text { cohort study. } \\
354,205 \text { birth } \\
\text { records in Sweden. }\end{array}$ & $\begin{array}{l}\text { - SGA rates identified by customised centiles showed a strong association with perinatal } \\
\text { mortality rates. } \\
\text { - Using customised centiles to determine SGA improves the identification of pregnancies at } \\
\text { increased risk of perinatal death. } \\
\text { - Customising for parity and maternal size results in a standard that has a substantially } \\
\text { strengthened association with perinatal mortality risk. }\end{array}$ \\
\hline
\end{tabular}




\begin{tabular}{|c|c|c|}
\hline \multicolumn{3}{|c|}{ Studies arguing against using customised standards: } \\
\hline $\begin{array}{l}\text { (Zhang, et al., 2007) } \\
\text { The use of customised } \\
\text { versus population-based } \\
\text { birthweight standards in } \\
\text { predicting perinatal } \\
\text { mortality. }\end{array}$ & $\begin{array}{l}\text { Population based } \\
\text { cohort study. } \\
782,303 \text { births in } \\
\text { Sweden. }\end{array}$ & $\begin{array}{l}\text { - The increased risks associated with SGA babies classified by customised standards is due to the } \\
\text { inclusion of a large proportion of preterm births, as customised standards will classify a higher } \\
\text { proportion of preterm infants as SGA. }\end{array}$ \\
\hline $\begin{array}{l}\text { (Ego, et al., 2008) } \\
\text { Should parity be included } \\
\text { in customised fetal weight } \\
\text { standards for identifying } \\
\text { SGA babies? Results from a } \\
\text { French multicentre study. }\end{array}$ & $\begin{array}{l}\text { Retrospective } \\
\text { observational } \\
\text { study. } \\
51,126 \text { births in } \\
\text { France. }\end{array}$ & $\begin{array}{l}\text { - Adjusting for parity markedly decreases the proportion of primigravida with SGA babies, but } \\
\text { doesn't appear to improve the identification of high risk babies. } \\
\text { - Removing parity will simplify the Gardosi et al., model of customisation and remove the } \\
\text { assumption that lower birthweight babies for primigravida is normal. }\end{array}$ \\
\hline
\end{tabular}


The literature was overwhelmingly in favour of the evidence advocating for the use of standards based on customisation, rather than population based standards. But while the evidence for customisation exists, it has yet to be routinely applied in clinical practice.

\section{Putting the 'evidence' into practice}

Although the evidence exists to show that customisation improves the diagnosis of growth restriction, its use in practice remains limited (Maulik, 2006). There is often a time lag between evidence being produced and then incorporated into practice (Hanberg \& Brown, 2006). While using CGC is recommended by the PMMRC in $\mathrm{NZ}$, it is unknown how widespread their use is. NZ was one of the first countries to apply the evidence and develop a CBWC calculator specific to their population (McCowan, et al., 2004). The DHB in this study, which implemented CBWC, understood they were the only DHB in NZ calculating a CBWC for every birth. The time taken for CBWC to be put into practice at this DHB in demonstrated in Table 2. It took 17 years from when CGC were first developed for CGC to be recommended by the PMMRC. It also took 12 years for a CBWC calculator to be developed for a NZ population. From this point, it took an additional three years for the DHB to use CBWC, which was not long after it was discussed as part of a HDC case.

Table 2. Timeline for putting the evidence behind CBWC into practice at one NZ DHB

\section{Timeline Milestone}

Customised antenatal growth charts developed (Gardosi, et al., 1992).

CBWC developed for a NZ population (McCowan, et al., 2004).

HDC report expressing concern over DHB's not using CBWC (Health

and Disability Commissioner, 2007).

CBWC initially implemented at the DHB in the study.

Guidelines and a flowchart incorporating CBWC finalised at the DHB in the study, after much debate.

First recommendation by PMMRC to complete CGC for every pregnancy in NZ (PMMRC, 2009).

\section{Publication date}

February 1992

October 2004

September 2007

October 2007

March 2008

March 2009 
CBWC for an Australian population was first published in April 2007 (Mongelli, Figueras, Francis, \& Gardosi, 2007). CGC developed for a US population was first published in July 2009 (Gardosi \& Francis, 2009b). This shows that the NZ and the DHB in this study were ahead of other countries in developing their own customised standards and then applying them in practice.

\section{Summary}

The GROW software determined the degree of influence a number of physiological variables of pregnancy had on birthweight. This software was then used to create customised growth charts (CGC) and customised birthweight centiles (CBWC). Fundal heights are plotted on CGC and identify growth restriction antenatally by predicting birthweight. CBWC are calculated at birth using the actual birthweight. Using CBWC versus sex-adjusted population centiles will result in picking up SGA and LGA babies who previously would have been missed and decreases the number of false positives. Customisation will result in identifying babies that are pathologically rather than physiologically small or large. The majority of the studies showed using customised standards are a more accurate way of identifying babies that are truly growth restricted. And babies identified as SGA by CBWC only, have the highest rates of perinatal morbidity and mortality. Nevertheless, this evidence hasn't been put into practice to the degree recommended. The next chapter will discuss knowledge translation theory which is about putting knowledge into action. It then introduces a conceptual knowledge translation framework which was used as a research tool for this study. 


\section{Chapter Three: Knowledge Translation}

\section{Introduction}

A CBWC calculator developed for a NZ population has been available since 2004 (McCowan, et al., 2004). However, a 2007 HDC case highlighted the fact that this evidence has not been put into practice in NZ. The Commissioner asked why a gap existed between the evidence available and what was seen in clinical practice. This chapter discusses knowledge translation theory, which is related to putting the action back into evidence based practice. The knowledge-to-action (KTA) framework is introduced which illustrates the ideal phases required for knowledge translation to occur. Frameworks can help us to make sense of complex processes. Therefore, the KTA framework was selected to help describe the processes involved with the implementation of CBWC at the NZ DHB studied.

\section{Knowledge Translation}

A unpublished literature search conducted by McKibbon and colleagues, found more than 90 different terms to describe how research is used (McKibbon, 2009, as cited in Straus, Tetroe, \& Graham, 2009). Terminology such as: knowledge uptake, implementation science and practice change are used. But the term knowledge translation is increasingly used today. Knowledge translation is defined as:

A dynamic and iterative process that includes the synthesis, dissemination, exchange and ethically sound application of knowledge to improve health, provide more effective health services and products, and strengthen the health care system. (Straus, Tetroe, \& Graham, 2009, p. 165)

Knowledge translation occurs in response to curiosity and is about problem solving with a purpose (Landry, Amara, Pablos-Mendes, Shademani, \& Gold, 2006). It uses the best knowledge available (World Health Organization, 2006), which is evidence 
based. The concept of knowledge translation promotes a culture of learning, critical thinking, innovation and change. It recognises that moving from knowledge to action is not a one-way process (World Health Organization, 2006). Knowledge translation is not a 'state' or 'end product' and is constantly changing (Graham \& Tetroe, 2009). It depends on people and context (Landry, et al., 2006) and the culture of the organisation in which the knowledge is being applied (Graham \& Tetroe, 2009). Knowledge translation cannot happen on its own. It "requires careful planning, implementation and monitoring with people who are engaged in and committed to change" (Graham \& Tetroe, 2009, p. 158). Also integral to its success is the need to consider the various stakeholders or end-users of the knowledge being implemented (Straus, Tetroe, \& Graham, 2009). The key elements required for knowledge translation have been put together visually by Graham et al., (2006), in a knowledge-to-action (KTA) framework (Figure 4).

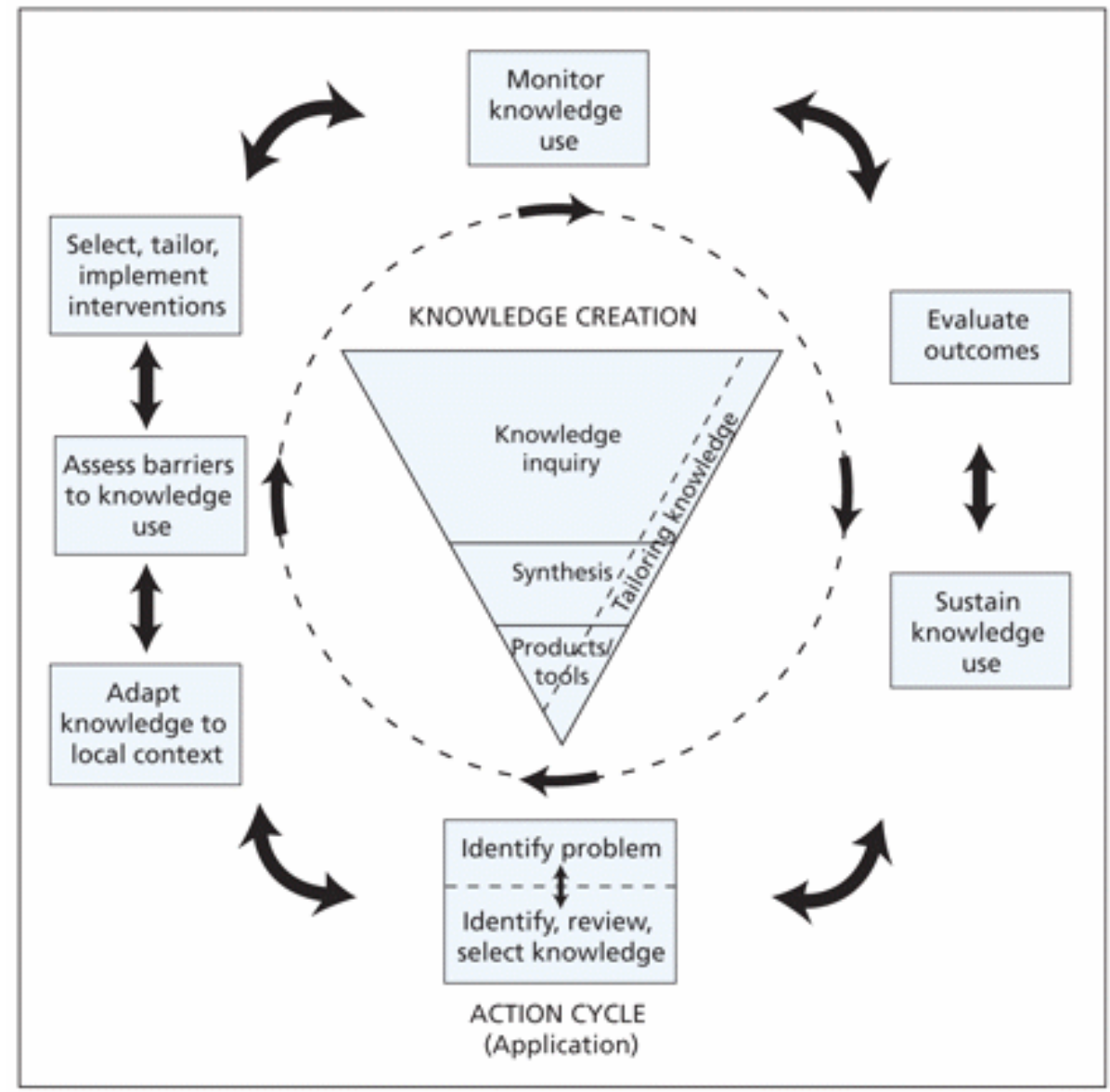

Figure 4. The knowledge-to-action framework (Straus, Tetroe, \& Graham, 2009, p. 167) 


\section{The knowledge-to-action (KTA) framework}

The KTA framework was first published in 2006 by Graham et al. (2006). The framework was developed from the similarities found in reviewing planned action theories (Straus, Tetroe, \& Graham, 2009). It was created to help make sense of the phenomenon of knowledge translation, by providing a map to guide the application of knowledge into action (Graham \& Tetroe, 2010). The authors believed that "given the action cycle's grounding in planned action theory, the framework can be considered evidence-informed" (Graham \& Tetroe, 2010, p. 212). They also acknowledged they were unaware of any studies evaluating the framework itself.

The framework (Figure 4) illustrates the two processes of knowledge creation and knowledge application. The middle funnel symbolises knowledge creation which involves the phases of knowledge inquiry, synthesis and products/tools. The action cycle on the perimeter represents the phases related to knowledge application. These are: identify the problem/identify review, select knowledge, adapt knowledge to local context, assess barriers to knowledge use, select, tailor and implement interventions, monitor knowledge use, evaluate outcomes and sustain knowledge use. It depicts movement and flexibility within the framework by using double sided arrows. While appearing linear, in reality the knowledge translation process is complex and each phase can inform other phases. This can happen sequentially or simultaneously, as the boundaries between knowledge creation and knowledge application are fluid and permeable (Graham \& Tetroe, 2009).

\section{Knowledge Creation}

As knowledge moves through the funnel, it becomes more refined and useful as it is tailored to meet the needs of the end-users of the knowledge (Brouwers, Stacey, \& O'Connor, 2010). The knowledge creation funnel is divided into three interrelated parts. Knowledge inquiry relates to the completion of primary research (Straus, Tetroe, \& Graham, 2009). It is also known as first-generation knowledge which is derived from primary studies, for example the results of a randomised control trial (Brouwers, et al., 2010). Synthesis is about focusing on whether there is a reliable 
and valid base of evidence that exists (Brouwers, et al., 2010). The findings that exist on any given topic are brought together and the quality of the evidence is considered (Straus, Tetroe, \& Graham, 2009). This involves looking at the evidence as a whole, rather than relying on individual studies. This synthesis of evidence is also known as second-generation knowledge and provides the basis for tools such as decision aids, guidelines and policies (Brouwers, et al., 2010). The development of a product or tool is known as third-generation knowledge (Brouwers, et al., 2010). This presents the knowledge in a user-friendly format for stakeholders, which can help facilitate the uptake of knowledge (Graham, et al., 2006). To increase the likelihood of implementation, ideally, a multi-disciplinary group would be engaged with the development and endorsement of the tool (Brouwers, et al., 2010). Strategies then have to be put into place to ensure that this knowledge passes through each of the phases of the action cycle.

\section{Knowledge Application (Action Cycle)}

The cyclical part of the framework represents the phases by which knowledge is implemented (Straus, 2009). This requires a proactive effort to encourage the use of knowledge at the decision-making level (Harrison, Legare, Graham, \& Fervers, 2010). The action cycle is divided into seven interrelated parts (Figure 4 p. 29) which are discussed next.

The action cycle begins by first identifying a problem that requires addressing (Graham, et al., 2006). Knowledge or research relevant to that problem is then selected (Doran, 2010) and the local and social context are considered (Kitson \& Phil, 2009). Customising knowledge to a particular organisation can improve compliance, especially when there is active involvement of the end-users (Harrison, et al., 2010). Part of this process includes identifying people who can help, support or facilitate this change (Graham, et al., 2006). The uptake of evidence and adapting to change is closely associated with assessing barriers to knowledge uptake (Harrison, et al., 2010). When barriers have been identified, then specific interventions should be linked to each barrier and tailored in a way to overcome them (Wensing, Bosch, \& Grol, 2010). Examples of different types of interventions 
are: professional interventions (educational programmes, decision support tools), passive educational interventions (guidelines, conferences), active educational interventions (websites for self-study), organisational interventions (enhanced teamwork, revision of roles), patient-directed interventions and financial interventions. But there is a lack of evidence around which is the most effective type to use (Wensing, et al., 2010). However what is known is that any intervention that employs only one technique is unlikely to produce a change in performance (Davis \& Davis, 2010). Monitoring the uptake of knowledge is another important aspect of the application process.

The extent to which knowledge is being used needs to be measured (Straus et al., 2010). Strategies employed to measure knowledge uptake may vary according to the group being monitored. For example, when assessing policy-makers you may use interviews and document analysis. When assessing physicians, you may use clinical databases, or when assessing patients, surveys may be used (Straus, et al., 2010). When the degree of knowledge use is found to be less than expected, it may be necessary to reassess the barriers to knowledge use (Straus, et al., 2010). It is also important to measure whether the new knowledge is actually making a difference and having the intended impact. Evaluating outcomes involves determining whether the knowledge being applied is actually improving health outcomes and whether the effort required for implementation is worthwhile (Graham, et al., 2006). Monitoring outcomes may also result in finding unintended consequences of a new intervention (Straus, et al., 2010). Ideally, outcomes should be measured at the patient level. But determining outcomes at the patient level can be a prohibitively expensive exercise to undertake (Straus, et al., 2010). This often means outcomes are not evaluated properly and in turn there is no sustaining of knowledge use. Sustainability is "the degree to which an innovation continues to be used after initial efforts to secure adoption is completed" (Rogers, 2005, p. 429). Sustainability is also "when new ways of working and improved outcomes become the norm" (Maher \& Evans, n.d, p. 2). The sustainability phase is supposed to set in motion a feedback loop that cycles itself through the action phases of the framework 
(Graham, et al., 2006). Little has been written about this aspect of the KTA framework even though it is an integral part of the knowledge translation process. The application cycle is intended to be a continuous ongoing process and this is achieved by sustaining knowledge use.

\section{Why use this framework?}

A framework organises a set of coherent ideas, so they can be more easily communicated to others (Doran, 2010). It is helpful for health professionals to learn about theoretical frameworks so they can understand how to increase the uptake of knowledge and influence change in their practice setting (Graham, et al., 2006). Closing the research-practice gap is a complex endeavour and a conceptual framework can help with understanding this process better (Graham \& Tetroe, 2007). When using any framework, documenting your experiences can enhance the understanding of others wanting to use it (Graham \& Tetroe, 2007). So it is acceptable for researchers to comment on the particular contribution a framework makes.

Knowledge translation is a field in its infancy with most commentary in the form of opinion pieces and anecdotal reports, with limited formal evaluation having been done (Mitton, Adair, McKenzie, Patten, \& Perry, 2007). It has been acknowledged by its authors that the KTA framework "has been widely cited, but has not, as yet, been tested empirically" (Graham \& Tetroe, 2010, p. 207). However, ten of the theories used in designing the framework had been empirically tested. The authors were "unaware of studies that have been specifically designed to evaluate the KTA framework" (Graham \& Tetroe, 2010, p. 216). There are only a few examples in the literature where the framework has been used in strategy or project development. In one example the framework was used as a model for the development of a mentorship strategy (Straus, Graham, Taylor, \& Lockyer, 2008). In another example the framework was used to design a project on preventing delirium after a hip fracture (Straus \& Holroyd-Leduc, 2008). More studies evaluating the framework 
are needed so "future iterations of the KTA cycle will be informed by feedback from the researchers and knowledge-users who are trying to apply it" (Graham \& Tetroe, 2010, p. 207).

This thesis is timely and relevant, as there is a need for more research to be completed around evaluating knowledge translation processes. This study was not specifically designed to evaluate the effectiveness of the KTA framework. The main focus of this research was to evaluate how CBWC were implemented at one NZ DHB and the impact of utilising CBWC. Early on it became apparent that the implementation process was complex. To be able to interpret what happened and describe the implementation processes in a meaningful way the KTA framework was selected retrospectively as a research tool to organise the data collected. By using the framework in this way I was then able to comment on its usefulness when applied to the clinical practice issue of implementing CBWC.

\section{Summary}

Knowledge translation is about putting knowledge into action. The process itself is complex and using a framework can aid in understanding the dynamics involved. The KTA framework conceptualises knowledge translation theory by depicting the ideal phases that should occur. It illustrates that implementing change and incorporating new evidence is an ongoing process. The ultimate purpose of knowledge translation is to improve health outcomes and service delivery. This is measured by evaluating whether the knowledge is used as it was intended and whether it improves clinical outcomes. This research study is about evaluating how the evidence behind CBWC was implemented at one NZ DHB and the outcomes resulting from this change in practice. The following chapter describes the overall study design and methods used and provides the justification for why an evaluation research approach was chosen. 


\section{Chapter Four: Research Approach and Design}

\section{Introduction}

The setting for this study is a maternity unit at one NZ DHB, which calculates a CBWC for every birth. The DHB understood they were the only hospital in NZ using CBWC to identify SGA and LGA babies and their subsequent risk of hypoglycaemia. The hospital provides secondary care maternity services for a large Maori birthing population and has 700 to 750 births per year. Having previously worked at the DHB I was familiar with the maternity service structure. I was aware the DHB had wanted to evaluate any outcomes since implementing CBWC, but hadn't had the opportunity to do so. This study was designed to answer this question and other questions concerning the implementation of CBWC at the DHB. This chapter outlines the approach and design selected to answer the overall research question and objectives. It provides the rationale for choosing this approach and describes the methods used to achieve this evaluation. As well, it describes the systematic way in which the data was collected and analysed and discusses some of the ethical issues involved.

\section{Research approach and design}

This study uses a mixed method evaluation approach to answer the overall research question which was:

How was the evidence behind the use of CBWC put into practice at one $N Z D H B$ and what are the outcomes resulting from translating this knowledge into action?

The first part of the research question asks; 'How was the evidence behind the use of $C B W C$ put into practice at one $N Z D H B$ '. To answer this required collecting qualitative data to gain the perspectives of individuals involved with implementing CBWC. The second part of the question asks; 'What are the outcomes resulting 
from translating this knowledge into action'. Answering this part would require the collection of quantitative data to evaluate outcomes resulting from this change in practice.

Several objectives were developed by dissecting the research question into smaller parts. Examining each part then contributed to answering the research question as a whole. The objectives chosen to answer the overall research question were:

- To identify and critique the evidence relating to the use of CBWC

- To describe how CBWC were implemented at one NZ DHB

- To evaluate the processes followed when utilising CBWC

- To identify outcomes relating to babies identified as SGA or LGA using CBWC

To adequately answer the objectives a variety of qualitative and quantitative methods were needed, so a mixed methods design was chosen. The study was about evaluating the experiences of people and events that occurred as CBWC were implemented and the extent to which the processes of implementation were being followed correctly. The study was also about evaluating some of the outcomes that resulted from introducing CBWC. Therefore a process-outcome evaluation approach was chosen.

Ethical approval to conduct this study was granted by the NZ Northern Y Regional Ethics Committee (see Appendix A). Approval from the DHB where the research was conducted was also received. Support was given from the Pouwhakahaere Hauora Maori (Maori Health Manager) and written approval was granted by the Chairman of the Clinical Board (see Appendix B). 


\section{The value in using a mixed method evaluation approach}

Evaluations are often conducted in the healthcare setting as they can help to improve programmes and assist decision making around whether a treatment or procedure should continue (Brophy, Snooks, \& Griffiths, 2008). Evaluations can highlight both the positive and negative impacts of an intervention, treatment, service or programme. Even services which are implemented with the best possible intention, may actually result in people being worse off than when they started (Brophy, et al., 2008). The most effective healthcare programme would be one that is designed to have some kind of evaluation framework incorporated in it (Brophy, et al., 2008). This would allow for the programme to be evaluated at a later date. Programmes need to be evaluated to see if it was implemented as planned and whether it is producing the intended outcomes. Using mixed methods in an evaluation can help to "provide a more comprehensive view of the outcomes" (Datta, 2001, p. 33). Using both qualitative and quantitative methods is especially valuable when evaluating complex interactions (Campbell et al., 2000).

\section{Evaluation requirement of the study}

Evaluation can be considered a research methodology when the data is collected and analysed in a systematic way (Patton, 2002). Evaluation research is usually investigator initiated and theory based, with the evaluation usually being the object of the study (Bickman, as cited in Patton, 2008). This evaluation is being performed to fulfil the requirements for a Masters Thesis. In this context, this evaluation can be considered research as it is being conducted in a systematic way, initiated by myself and is based on the theoretical concept of knowledge translation. 
A comprehensive definition of evaluation from the 'Encyclopaedia of Evaluation' states:

Evaluation is an applied inquiry process for collecting and synthesizing evidence that culminates in conclusions about the state of affairs, value, merit, worth, significance, or quality of a program, product, person, policy, proposal, or plan. Conclusions made in evaluations encompass both an empirical aspect (that something is the case) and a normative aspect (judgment about the value of something). It is the value feature that distinguishes evaluation from other types of inquiry. (Deborah Fournier, as cited in Mathison, 2005, p. 139)

The empirical aspect of this evaluation is in illustrating how the evidence behind CBWC was implemented. The normative aspect is in making a judgment about the value of CBWC. Patton (2008) adds that by evaluating a programme, you can make judgments and develop an increased understanding of a programme. In simple terms, the way evaluation differs from other types of inquiry is that it measures the value of something. This simplistic definition demonstrates the broad scope of evaluation. This provides the researcher with the ability to look at almost anything in a meaningful way by using appropriately selected methods. As a result, evaluations are often categorised by type or focus.

\section{Evaluation types or focus}

Patton (2008) provided a table which lists 79 different evaluation focuses or types and explains that the list doesn't exhaust all the possibilities. More than one type may be used within an evaluation or others may be implemented over time as the focus or defining question of the evaluation changes. The three most common types of evaluation are: outcome evaluation, process evaluation and summative evaluation (Table 3). The type chosen will depend on the defining research question or research approach selected. 
Table 3. Ways of focusing Evaluations

\begin{tabular}{ll}
\hline \multicolumn{1}{c}{ Focus or Type of Evaluation } & \multicolumn{1}{c}{ Defining Question or Approach } \\
\hline Outcomes evaluation & $\begin{array}{l}\text { To what extent are desired client/participant } \\
\text { outcomes being attained? What are the effects } \\
\text { of the programme on clients or participants? }\end{array}$ \\
Process evaluation & $\begin{array}{l}\text { Evaluating the activities and events that occur as } \\
\text { part of implementation: What do participants } \\
\text { experience in the programme? What are the } \\
\text { strengths and weaknesses of day-to-day } \\
\text { operations? How can these processes be } \\
\text { improved? }\end{array}$ \\
Summative evaluation & $\begin{array}{l}\text { Should the programme be continued? If so, at } \\
\text { what level? What is the overall merit and worth } \\
\text { of the programme? }\end{array}$ \\
\hline
\end{tabular}

Adapted from (Patton, 2008, p. 300).

An outcome evaluation is usually conducted retrospectively on established programmes, so sufficient time has lapsed for the program to cause an effect (Owen, 2006). A process evaluation "looks at how the intervention is formed or how the processes of the intervention come together, and focuses on the ways that a programme or intervention is working" (Brophy, et al., 2008, p. 27). Sometimes these two types are combined to then become a process-outcome evaluation. This evaluation will measure the degree of implementation, as well as determining outcomes. This is often necessary because the outcomes might be better explained once the extent to which the programme has been implemented is understood (Owen, 2006).

\section{Applying a mixed method evaluation approach}

Performing a mixed methods process-outcome evaluation is appropriate as both qualitative and quantitative data is required to answer the research question and objectives. 
Patton (2002) lists four ways that qualitative inquiry is appropriate when trying to understand the dynamics of how a programme or organisation works:

1) depicting process requires detailed descriptions for how people engage with each other

2) the experience of process typically varies for different people so their experiences need to be captured in their own words 3) process is fluid and dynamic so it can't be fairly summarized on a single rating scale at one point in time

4) participants' perceptions are a key process consideration (p. 159)

Understanding and describing these dynamics can help you decide what elements may have contributed to the success or failure of the programme (Patton, 2002). Quantitative inquiry in evaluation is often used when "selecting measures for the intended programme outcomes and assessing the extent to which changes in the outcomes for the programme's target population can be attributed to the programme" (Jennifer Greene \& Gary Henry, as cited in Mathison, 2005, p. 348). To understand the processes involved with implementing CBWC at the DHB, a mixed method evaluation was required.

Mixed methods research in evaluation has been defined as:

... one that planfully juxtaposes or combines methods of different types (qualitative and quantitative) to provide a more elaborated understanding of the phenomenon of interest (including its context) and, as well, to gain greater confidence in the conclusions generated by the evaluation study. (Valerie Caracelli as cited in R. Johnson, Onwuegbuzie, \& Turner, 2007, p. 119)

By using different methods to gather different kinds of data, the validity of the study increases through the use of triangulation. Triangulation increases certainty in the results by using methods which can cancel out each other's limitations (Datta, 2001). Triangulation in evaluation can also enhance "the validity or credibility of evaluation findings through results from the different methods that converge and agree one with the other" (Jennifer Greene, as cited in Mathison, 2005, p. 255). Mixed methods is commonly used in evaluation as it can help us to better understand 
"complex, multifaceted, real-world social phenomena" (Jennifer Greene, as cited in Mathison, 2005, p. 255). Using both qualitative and quantitative methods is particularly helpful when evaluating complex knowledge translation interactions (Straus, et al., 2010).

In determining the best way to use mixed methods to evaluate the implementation process, I realised I needed to support my description of events from a theoretical perspective. The literature was examined to determine what theories might be useful to help answer my research question (Creswell, 2009). I explored knowledge translation theory, which is about putting the action back into evidence based practice. I examined the knowledge-to-action (KTA) framework which described the ideal phases required for knowledge translation to occur. I decided to apply the KTA framework retrospectively as a research tool to help me describe what happened in a meaningful way.

\section{Using the KTA framework as a research tool}

The KTA framework was used to provide a theoretical rationale to explain how each implementation process was interrelated. Using a framework to evaluate an implementation process provides a holistic account of what happened by showing multiple perspectives and identifying the various factors involved (Creswell, 2009). The funnel shaped section of the framework relates to knowledge creation. These steps occurred as a result of the research that went into the development of the CBWC calculator. This is described in Chapter Two which discusses the evidence supporting the use of CBWC. The circular part of the framework relates to knowledge application. In learning more about how the framework depicts the knowledge translation process, the action cycle seemed to naturally divide itself into two parts (Figure 5). The first four headings of the action cycle (shaded green) related to the phases involved with the actual implementation process. This part would be best described using mainly qualitative data. The last three headings (shaded red) were about measuring the extent of implementation. This part would be best explained using mainly quantitative data, but qualitative data could also be used to further validate the quantitative findings. 


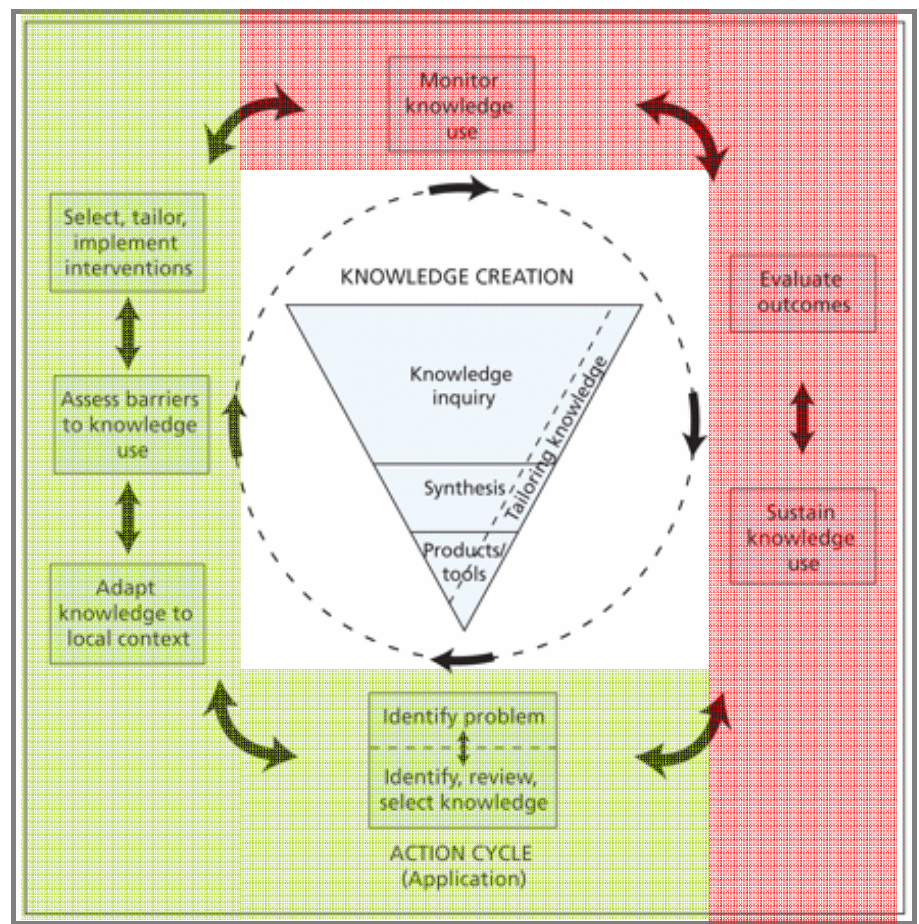

Figure 5. The 'coloured' knowledge-to-action framework (Straus, Tetroe, \& Graham, 2009, p. 167)

Retrospectively applying the KTA framework to a clinical practice issue provided an opportunity to comment on the usefulness of the framework in this setting. An additional research objective was then added to the previous objectives. This objective did not aid in answering the overall research question, but provided insight into the useability of the KTA framework which was:

- To evaluate the usefulness of the knowledge-to-action framework when applied to a clinical practice issue

The KTA framework provided a template to be overlayed on top of what happened at the DHB, which then described the implementation process in an organised way. The methods selected provided the data embedded within the framework, which related the knowledge translation story. 


\section{Methods used to achieve this evaluation}

The purpose of an evaluation influences the design and methods chosen. It is important that the methods chosen for data collection and analysis are the ones which will provide the best answers to the questions (Owen, 2006). The resources available also need to be considered as you will be constrained by time, finances and expertise (Brophy, et al., 2008). This relates to considering the rigour and feasibility of the evaluation. Rigour involves making the evaluation strategy explicit and using valid methods. Feasibility relates to keeping the evaluation strategy realistic and appropriate within the setting (Straus, et al., 2010). This was small scale research project conducted to fulfill the requirements for a Masters Thesis, which limited the size and scope of the research.

Interviews, focus groups, a clinical audit and document analysis were the methods chosen to answer the objectives and overall research question. They were conducted in this order as the data collected from each method informed the next method used. Considering the relationships between the types of data collected influences the order and combination of methods (Saks \& Allsop, 2008). By performing the audit after the interviews and focus groups, the quantitative data did not influence how the qualitative data was collected. This ensured stakeholder perceptions of their experiences could be compared with what was really happening in practice.

\section{1) Interviews}

Interviews were chosen to gain insight from individuals in management positions as to why and how CBWC were implemented at the DHB.

\section{Size and method of sample}

In having some knowledge of the maternity service structure at the DHB, I identified who I believed were five key people to interview. These were the; Midwifery Educator, Maternity Unit Manager, Neonatal Quality Coordinator, Clinical Director of Women's Health and Clinical Director of Child Health. Letters of information (Appendix C) and consent forms (Appendix D) were mailed, inviting them to be 
interviewed. I did not receive any response when trying to contact the Clinical Director of Women's Health. The Midwifery Educator informed me that the Clinical Director of Women's Health was difficult to contact and was not really involved with implementing CBWC. Instead she suggested interviewing a particular midwife who had been vocal about the changes. This midwife was contacted to be interviewed instead. An interview with the Clinical Director of Child Health was also not achieved after several requests to do so. In the end, four interviews were completed.

\section{Method of obtaining data}

The Midwifery Educator was interviewed first, as she was the person most familiar with the implementation process. This interview took the longest, but provided the greatest insight. Next the Maternity Unit Manager, midwife and Neonatal Quality Coordinator were interviewed as they were available. The interviews lasted between 30 to 75 minutes and were recorded. An interview schedule of basic questions was used (Appendix E). Each interview informed subsequent interviews and specific questions were formulated relating to that individual's role in the process. The interviews also helped to refine the focus group questions. I felt the interviews were successful as I was provided with the information needed to help answer the relevant research objectives. Each interviewee was open and forthcoming with their responses and some came prepared with documentation to validate what they remembered. Each participant expressed that they looked forward to hearing the results of the research. Interviewees were given a gift voucher as a thank you for their participation.

An interview is a "conversation with a purpose", where specific topics are discussed in-depth (Hennink, Hutter, \& Bailey, 2011, p. 109). One-to-one semi-structured interviews are the most common qualitative method employed and is known as the 'gold standard' approach (Barbour, 2008). They are often "used when seeking information on individual, personal experiences from people about a specific issue 
or topic" (Hennink, et al., 2011, p. 109). Semi-structured interviews were used as key people were known to be involved with the implementation of CBWC.

\section{Ethical considerations}

The rights of participants were considered by giving letters of information which fully disclosed what the study involved and emphasised voluntary participation. It was important to maintain a balance between attempting to commit people to being part of the research and allowing people the right to decline to participate. Confidentiality forms were signed before the interviews commenced.

Anonymity was maintained by not naming the DHB in the research. For this reason demographics describing the setting are intentionally broad, including non-specific ethnicity statistics. NZ is a small country and the DHB may have been identifiable if these statistics were used, as well as the interviewees. Interviewees were aware they may be identified by their job title, but that they would not be individually named.

Ethical consideration was needed in considering my personal reflexivity as a researcher. This involved acknowledging and examining my own characteristics, biases and insights and how they might influence the participants, the evaluation process and the findings (David Williams, as cited in Mathison, 2005). I was known by all the participants and this could have potentially indicated a conflict of interest and had an impact on the findings. But I felt this wasn't a hindrance as participants willingly shared information with me, as a rapport was already established. I wasn't working at the maternity unit at the time $\mathrm{CBWC}$ were implemented so I was unaware of the events that took place.

\section{2) Focus Groups}

Focus groups were chosen to gain an understanding of the end users perspective of how $\mathrm{CBWC}$ were implemented and how they were being used in practice. 


\section{Size and method of sample}

Two focus groups were chosen as there were two distinct end user groups involved with calculating the CBWC and using it in practice. One focus group was for LMCs (lead maternity caregivers). These were self-employed community midwives that provided primary antenatal, labour, birth and postnatal care services. Another focus group was for core staff (midwives and/or nurses) employed by the hospital who provided secondary care services for women and their babies. Letters of information (Appendix F) and consent forms (Appendix G) were placed in the mail trays of all LMCs and core staff in the staffroom. Posters (Appendix H) advertising the focus groups were pinned in the office and staffroom. Reminders were emailed and texted to potential participants and some verbal RSVPs were received, but it was unknown how many would attend on the actual days.

\section{Method of obtaining data}

The focus group was moderated by myself and a brief question guide was used (Appendices I and $\mathrm{J}$ ) which provided an unstructured approach. In this way the discussion was facilitated rather than directed. The order of focus groups was important. The LMC focus group was held first and five from a possible eight LMCs attended. A week later the core staff focus group met and six people attended. The core staff focus group was held last as they are the ones who provide the ongoing management for babies identified as SGA or LGA by CBWC. The focus groups lasted 45 and 60 mins and were recorded. Within each group no one person dominated. Participants appeared to be comfortable with expressing their opinions, which lead to some enthusiastic and open discussions. Everyone was supportive of the research and looked forward to learning of the findings. Lunch was provided as a thank you for their participation.

A focus group gathers information from a selected small group of people through group discussion which aims to move from broader to more specific issues (Owen, 2006). This forum allows for the analysis of a complexity of views, rather than a single opinion (Saks \& Allsop, 2008). Participants are encouraged to talk to each 
other rather than just answering the moderators questions (Smithson, 2008). Separate focus groups were held to facilitate an open discussion within each homogenous group, as the LMCs and core staff played different roles in the implementation process.

\section{Ethical considerations}

The same principles of confidentiality, anonymity, rights of participants and my reflexivity as a researcher were considered for those participating in the focus groups. Participants were given letters of information and consent forms were signed before the focus groups commenced. Quotes used from the focus groups were identified as LMC or CORE, rather than LMC 1, LMC 2 etc as it was possible that people within the DHB may be able to identify individuals from their comments made. Quotes used from the individual midwife interviewed were included with the LMC quotes, as this person may have been identified by her colleagues if her comments were identified separately. Within the focus groups while the moderator can guarantee confidentiality with keeping the focus group discussions private themselves, they cannot guarantee that participants will not discuss the group outside the meeting (Smithson, 2008). Participants were reminded of the confidentiality agreement at the beginning, but this cannot be guaranteed outside the focus group.

\section{3) Audit}

An audit was chosen to evaluate if the correct processes were followed when calculating a CBWC and to identify outcomes for babies identified as SGA or LGA.

\section{Size and method of sample}

The maternity unit had approximately 700-750 births per year and 95\% of these births were at term ( $\geq 37$ weeks gestation). An online sample size calculator was used (www.raosoft.com/samplesize.html) to determine the audit number, accepting a $5 \%$ margin of error and a $95 \%$ confidence level. The recommended sample size was 249. There were approximately 55 term births per month. Auditing six months of births would include an estimated 330 term births, with additional babies eliminated once the final inclusion criteria was applied. It was decided that births from January, 
February, March 2009 would be audited, as well as births from November, December 2009 and January 2010. January 2009 was chosen as the starting point because this would have allowed enough time from when CBWC were implemented for LMCs to routinely collect maternal height and weight. January 2010 was chosen as the end point, so the audit was spread over a 12 month period. This meant a comparison could be made to see whether the audit results changed over time. Births included in the audit were those $\geq 37$ weeks gestation, but not including multiple pregnancies, stillbirths or congenital abnormalities, which was consistent with the methodology of other studies (McCowan, et al., 2004). The final audit sample size was 303 .

\section{Method of obtaining data}

The audit was conducted after the interviews and focus groups to prevent the findings influencing the interview and focus group process. The audit tool (Appendix K) initially included the woman and baby's NHI (National Health Index) number, date of birth and mother's initials for ease of referencing. This was later removed to anonymise the data for analysis. A pilot audit of 10 births, which was included in the final data set, was performed to estimate the time needed and to see whether the tool collected the required information. The birth register was used to fill in the tool as much as possible. The clinical notes were then used to complete the audit tool. Throughout this process the data was checked multiple times as it was entered on the audit tool spreadsheet. Each tenth entry was also systematically checked as the tool was being completed. The CBWC was computed using the centile calculator. Each CBWC calculation was re-checked multiple times. When the result was different from what had been recorded, or the result was borderline, or when a baby was identified as SGA or LGA, the result was checked several times again. Any relevant details were included in the comments column. Each CBWC was calculated individually rather than using the bulk calculator in order to simulate what happened in practice. 
The audit took much longer than expected due to the time required to request notes, re-check results, investigate discrepancies and fatigue. It was anticipated that only the maternal notes would need to be requested. But partway through the audit period the layout of the maternal and infant clinical notes changed, which meant I also had to request the infant notes as well. Instead of requesting over 300 notes I had to request more than 600 sets of clinical notes. A significant error was also discovered near the end of the audit when I realised I was using version 5.15 of the centile calculator and the maternity unit was using version 5.13. Version 5.15 was the most recent version which could be downloaded from www.gestation.net, but version 5.13 had not yet expired. I contacted the Perinatal Institute who advised the difference between the versions was that BMI limits were removed (A. Francis, personal communication, May 5, 2010). But this would most likely only result in small changes for very small or very large women (L. McCowan, personal communication, April 30, 2010). I recalculated the CBWC for each birth using version 5.13. In the majority of cases there was no change in the CBWC result. When the CBWC result did change, the difference was only small and there was no change in which babies were identified as SGA or LGA. Recalculating every CBWC was necessary as one outcome being audited was whether the CBWC I calculated was the same as what had been recorded. So it was essential that the same version of the calculator was being used.

An audit is "any summary of clinical performance of health care over a specified period of time" (Sudsawad, 2007, p. 16). An audit "compares actual practice to a standard of practice. As a result of this comparison, any deficiencies in actual practice may be identified and rectified" (Ministry of Health, 2002, p. 7). This is a relevant method to use when evaluating knowledge translation processes as audits can highlight implementation gaps and therefore priorities for knowledge implementation (Foy \& Eccles, 2009). 


\section{Ethical considerations}

When accessing the birthing register and clinical notes it was important that only the sections applicable to the research were examined. The birthing register was accessed at the maternity unit only and was never taken off-site. The clinical notes were accessed in the clinical records storage area and notes were never taken offsite. Once the audit tool was completed the data was then de-identified to protect anonymity. The NHI numbers, date of birth and maternal initials were removed. I was the only person to see the original raw data on the audit tool. The quantitative data analysis was performed using de-identified data only and all reporting of results and tables used de-identified data.

\section{4) Document analysis}

Document analysis was chosen in addition to the previous methods, to provide additional information to support the data to be collected.

\section{Method of obtaining data}

Documentation was used to corroborate and verify what had been revealed through the interviews and focus groups. For example, meeting minutes, emails, dated guideline drafts, guidelines and policies, letters and powerpoint presentations were used to verify dates and provide an accurate timeline of events. These were all examples of secondary data which were collected and recorded at an earlier time (B. Johnson \& Christensen, 2012) but was later used as part of this research. The alternative was to rely solely on people's memory recall of events, which likely would have resulted in inaccuracies in the data. Documentation was provided to me by participants to provide evidence for their comments. Other documentation was accessed in the maternity office which was freely available for all staff to read.

Documentation review is useful when you want to find comprehensive, historical unbiased information that already exists (Mertens, 2010). The documentation was able to give some of the "necessary background of the situation and insights into the dynamics of everyday functioning" (Mertens, 2010, p. 373). 


\section{Ethical considerations}

Documentation given to me was kept confidential and anything electronic was stored on my password protected computer, with hard copies kept in a locked safe. Documentation of a more sensitive nature which was accessible in the maternity office was left in its original location and only notes were taken. Only information relevant to the research, and in the timeframe being examined, was accessed.

Other electronically stored data (audit results, interview and focus group transcripts) were kept on mine and my supervisor's password protected computers and will be deleted after 10 years. Copies of the audit results, original recordings of the interviews and focus groups and signed consent forms are stored in a locked safe and will also be destroyed after 10 years.

\section{Data analysis}

The KTA framework was chosen as a template to illustrate the knowledge translation process and the data collected was positioned within this framework. Using it in this way was appropriate because a conceptual framework can help to guide, interpret and understand an implementation process and provides an organised way to think, observe and interpret what happens (Graham \& Tetroe, 2009). The headings in the framework provided the overall themes and thematic analysis of the qualitative data provided the subheadings. The quantitative data was analysed using descriptive statistics.

\section{Qualitative data analysis}

Using the KTA framework provided a map of the ideal phases occurring during a knowledge translation process. But this meant the data had to fit within the headings of the framework. Both pre-determined codes [headings in the framework] and emerging codes [codes developed from the data] (Creswell, 2009) were used. The analysis was deductive as it involved a process of coding the data then trying to fit it into a pre-existing coding frame (Braun \& Clarke, 2006). The data was interpreted and allowed to speak for itself but it was also structured so that the knowledge 
translation process was described in a systematic way. A basic generic form of qualitative data analysis was used which was to collect the data, analyse it for themes or perspectives and then report several themes (Creswell, 2009).

Immediately after each interview and focus group, initial impressions were noted. A table was constructed using the headings from the KTA framework to label each row. The tapes were listened to and points were typed under the heading it seemed to best fit. The process of transcribing tapes yourself is a good way to familiarise yourself with the data and develop a better understanding of the data (Braun \& Clarke, 2006). This allowed me to test whether my first impressions were supported by what was later heard on the tapes. When a noteworthy statement was heard this was transcribed verbatim. It is important to select compelling examples to illustrate the themes that emerge (Braun \& Clarke, 2006). Initially the table was also colour coded to identify who was speaking for ease of referencing, but the colour coding was later removed.

This was a labourious and confusing process as it was sometimes difficult to decide which heading was most appropriate to use. The process resulted in a large repetitive document. This was re-read many times and headings were regrouped to combine similar themes, by cutting and pasting within the table. Qualitative data analysis is a cyclical reflective process and is something you do with your data, rather than to your data (Carter, 2004). Qualitative data analysis is also iterative in that it is something that you repeatedly return to and it can be a complex and messy process (Carter, 2004). The coding was theory driven as there were specific predetermined headings that I wanted to use (Braun \& Clarke, 2006). One example of how codes emerged can be shown using the heading 'Assess Barriers to Knowledge Use', where there were initially 20 subheadings. 


\section{'Assess Barriers to Knowledge Use' initial codes}

1) New knowledge

2) Challenging guideline after it was signed off

3) Intervention on bigger babies

4) Accuracy of data

5) Installing the calculator

6) Mother/family resistant to change

7) No ongoing use

8) Not evidence-based

9) 'Normal' weight babies identified as SGA or LGA

10) Informing the mother

11) Missing data

12) Not following the guideline properly

13) Imposing your own beliefs on others

14) Time to accept it

15) Resistant to change

16) Fixing the results

17) Heelpricks

18) Interfering with breastfeeding

19) Whose role is it? Is it primary or secondary care?

20) Seeing the bigger picture

When I was unable to condense the subheadings any further within the document, I printed them onto strips of paper. The analysing process then became one of physically clustering and rearranging the subheadings over and over again. This was done until five final subheadings emerged which were:

\section{'Assess Barriers to Knowledge Use' final codes}

1) Resistance to changing practice

2) Using the CBWC calculator

3) Increased intervention

4) Following the guideline correctly

5) Primary or secondary care responsibility?

Halfway through the qualitative data analysis I realised I needed to use more direct quotes and I had to listen to the tapes again to transcribe more parts verbatim. I had thought my original transcribing was completed at an appropriate level of detail (Braun \& Clarke, 2006). But it turned out to be a mistake to have not originally transcribed the tapes word for word, as this would have saved me a lot of time. 


\section{Quantitative data analysis}

After completing the audit, data had been collected for 318 births. The data was

checked and some births were removed as they did not fit the inclusion criteria, which was; births $\geq 37$ weeks gestation, but not including multiple pregnancies, stillbirths or congenital abnormalities. The decision was also made to remove any birth that was recorded in the register as BBA (born before arrival). These were planned or unplanned homebirths where the mother and baby arrived at the hospital after the birth. These births were excluded because there was variation around whether the mother and baby were admitted to the hospital and whether care was handed over to the core staff. For example, one baby only had a few hours of transitional care in the NNU and then transferred home again. The baby was never admitted to the hospital so the baby's birth was incorrectly recorded in the register. Alternatively, another baby was admitted to the ward with its mother and care was handed over to the core staff. Because some of these births were incorrectly recorded in the register and there was variation in how old the babies were when they were admitted, all BBA births were excluded. The final audit number became 303 births.

Descriptive statistics relevant to the outcomes being evaluated were calculated and results were put into tables. Descriptive statistics function to "describe or indicate several characteristics common to the entire sample ... [and] summarise data on a single variable" (Mertens, 2010, p. 405). Using the SPSS statistical package was beyond the scope of this study and the audit number would not have been large enough to be able to determine statistical significance in the results. The quantitative findings were then placed within the appropriate heading in the KTA framework which was 'Evaluate Outcomes'.

\section{Demonstrating validity and reliability}

Research aims to be objective by demonstrating reliability and validity. Reliability is the extent to which a measurement procedure yields the same answer whenever it 
is carried out and validity is the extent to which it gives the correct answer (Kirk \& Miller, 1986). Commonly, qualitative research relies more on measures allowing validity, whereas quantitative research relies on checks for reliability (Kirk \& Miller, 1986). Using a mixed method approach which aims to combine "complementary strengths and nonoverlapping weaknesses of quantitative and qualitative research" is a contentious issue (Onwuegbuzie \& Johnson, 2006, p. 48). Combining methods to increase the overall validity of the research is a simplistic explanation for why it is used. But it can be said that using a mixed methods design is "one criterion for increased validity in a research study with multiple purposes and questions" (Mertens, 2005, p. 301).

Reliability is demonstrated by explicitly describing how the researcher collected and analysed the data (Kirk \& Miller, 1986). This was done in this chapter where the overall research design and methods used are described in detail. Reliability is also about a "tendency toward consistency found in repeated measurements of the same phenomenon" (Carmines \& Zeller, 1979, p. 12). The reliability of the audit tool could be measured if someone else was to complete the audit and achieve the same results. The calculator itself could be considered reliable in that when the same data is inputted it provides the same result. As this is a small scale piece of research, I did not use statistical tests to determine reliability.

Relating to qualitative data, validity is "a question of whether the researcher sees what he or she thinks he or she sees" (Kirk \& Miller, 1986, p. 21). Qualitative methods require a judgment to be made and will be influenced by an individual's background knowledge and understanding. Therefore, an element of nonvalidity will always be involved (Hammersley, 2008). Some also argue that the validity of research is tested by the application of the knowledge that is produced (Hammersley, 2008). The application of this knowledge is valid in the current NZ maternity system as the use of CGC has been recommended for all pregnancies by the PMMRC. The use of CBWC could be an extension of that recommendation. Generally, any measuring device can be considered valid if it does what it was 
intended to do (Carmines \& Zeller, 1979). The audit tool and question guides could be considered valid in that they answered the objectives of the study and overall research question.

\section{Summary}

Evaluation was an appropriate research approach to select because of its characteristic broad scope. This allowed a variety of methods to be employed to answer the study objectives, which in turn answered the overall research question. The use of a process-outcome evaluation meant that the process of implementation and the outcomes resulting from this change in practice could be examined. A mixed methods design was needed to provide a better understanding of the complex processes involved, while also strengthening the validity of the research. Using the KTA framework provided a way to discuss the findings within a theoretical context and ethical principles were adhered to throughout the research. The following chapter describes in detail the results of the study using the qualitative and quantitative data collected. This 'knowledge translation story' is told using the KTA framework as a template to outline the implementation process that took place. 


\section{Chapter Five: Results}

\section{Introduction}

Making sense of the processes that occurred as CBWC were implemented at the DHB studied, was more complex than expected. For this reason a knowledge translation framework was chosen to help organise the collected data in a meaningful way. This chapter presents the results of the 'knowledge translation story' that occurred as the evidence behind CBWC was put into action at one NZ DHB. The KTA framework is used as a template which provides the story outline and the data is positioned within the headings of the framework. The knowledge creation funnel is not included as part of the results, as Chapter Two provides the background and evidence for using CBWC. Therefore this chapter tells the story relating to the 'action cycle', which is about how the knowledge was implemented and put into action. The first part of the story describes how CBWC were implemented using qualitative findings and the second part talks about measuring the extent of implementation using mainly quantitative findings. Qualitative findings are also used to verify the outcomes of the audit.

\section{Part A: The implementation process, the story begins ...}

The phases of the action cycle shaded green (Figure 5, p. 42) were involved with the actual implementation process. These were; identify the problem/identify review, select knowledge, adapt knowledge to local context, assess barriers to knowledge use, select, tailor and implement interventions. This part of the story is told from the perspective of the senior management involved with implementing $\mathrm{CBWC}$, as well as the perspective of the staff and community midwives who were expected to abide by the appropriate guidelines and calculate a CBWC after every birth. Practitioners were unfamiliar with $\mathrm{CBWC}$ and were challenged by having to use them as a method of identifying babies at risk of hypoglycaemia. Many barriers existed to prevent individuals from applying the evidence into their practice. The importance 
of a champion was identified who maintained the momentum needed for CBWC to be put into practice. She employed numerous interventions to progress the knowledge translation process beyond the knowledge creation stage.

In this part of the story, 'Assess barriers to knowledge use' is discussed last, which is in a different order from the KTA framework. The KTA framework was not used by the DHB implementing CBWC but was used by myself in making sense of the data. So barriers were not identified before the implementation process began. Instead the barriers were identified by myself as part of the qualitative data analysis. The authors of the framework have explained that the phases do not have to be sequential (Graham \& Tetroe, 2009)

Extracts used to tell this story can be identified by the following key.

\section{Key for interview and focus group extracts:}

Midwifery Educator: Midwifery Educator/Quality Coordinator MM: Maternity Unit Manager NNU: Neonatal Unit Quality Coordinator

LMC: Lead Maternity Caregiver (self-employed community midwives) and individual midwife interviewed

CORE: Core staff midwife or core staff nurse

\section{1) Identify problem $\leftrightarrow$ Identify, review, select knowledge}

At a small DHB, individuals often have multiple roles within the organisation that would be staffed by a number of employees at larger DHBs. For example larger DHBs often have more than one Midwifery Educator whose sole role is to coordinate ongoing midwifery education needs. The Midwifery Educator in the study had many responsibilities, including updating and formulating evidence based guidelines in her additional role as the Quality Coordinator and acting as the local Perinatal Maternal Mortality Review Committee (PMMRC) Coordinator. In this role, she learned that the PMMRC were calculating CBWC for all perinatal deaths nationally, which was the first time she had heard of CBWC. 
That's what the PMMRC do and have done since July 2006. So for any perinatal death they are collecting $C B W C$. So I sort of looked at that, I didn't really know what it was before then. (Midwifery Educator)

At the time she was only able to locate sex-adjusted birthweight centile charts and decided to investigate what CBWC were. She was introduced to GROW and began looking up the evidence around CGC and CBWC by Professor Jason Gardosi at the Perinatal Institute in the UK. She then liaised with the National PMMRC Coordinator and Associate Professor Lesley McCowan, who did the NZ research around CBWC. She learned that CBWC weren't being used by hospitals and became excited that the DHB could be forward thinking by calculating a CBWC for every birth. From examining the evidence, she felt that CGC and CBWC would be beneficial tools for the maternity unit to use and would help practitioners to better identify IUGR babies antenatally and SGA babies postnatally. She felt as a DHB they could be providing better care for their 'high risk' birthing population and that babies were probably being missed.

The next step was for her to introduce the evidence to other practitioners and convince them of the benefits. She gave a presentation at a perinatal meeting in July 2007 introducing the concepts around CGC and CBWC. The majority of practitioners had never heard of CBWC, including midwives who had recently worked in the UK.

There was talk about it but they hadn't started using them when I left. I left June '08 they were talking about this new system they were going to bring in but it hadn't been implemented. (CORE)

CGC were implemented first but it took time for LMCs to incorporate these into their practice. CBWC were then included in the relevant guidelines, but could not be put into practice, as convincing IT technicians to download the calculator on the maternity unit computers proved difficult. 
You can download this package onto your home computer but of course all the computers in the hospital have limitations put on to what you download, so I had to give a sort of a business case for getting those on to the computers. (Midwifery Educator)

This obstacle was overcome when the findings of a HDC case relating to neonatal hypoglycaemia was released. This acted as a catalyst to allow the Midwifery Educator to get CBWC fully implemented.

The thing that really forged it ahead was the HDC case ... that came up which actually really helped me to implement that and to give a reason and rationale for doing it. (Midwifery Educator)

The HDC case released in Sept 2007 highlighted the problem of better identifying babies at risk of hypoglycaemia. This case was brought to the attention of the CEO as a learning tool for DHBs. The Clinical Director of Child Health was asked whether a similar scenario could happen at their hospital. The Clinical Director and the Midwifery Educator made a joint presentation to the Clinical Board on what the maternity and neonatal unit had put in place to prevent a case such as this.

We had to present a report on how we think it is unlikely to happen here or what we are putting in place and this was one of those things, the $C B W C$. (Midwifery Educator)

Some felt the HDC findings had generated an exaggerated response from management.

There's this absolute fear. Can I just say that it's fear of litigation too. There's a lot of defensive practice as to why we implement things like this too. Not on my shift, not in my hospital ... I've heard it out of people's mouths at the top management. (LMC)

Once the tool was available on the computers, people were instructed on its use and which guidelines to follow. It was at this point that the main debate about the issues began, as people were challenged by the reality of calculating and using CBWC in their practice. 
[The Clinical Director of Child Health] would say look this is your guideline, it's just signed off. You had a chance to debate it and they didn't actually when it was going through. Only when it had gone through then afterwards they suddenly [did]. (Midwifery Educator)

A UNICEF guidance document on hypoglycaemia was also published in December 2007. This initiated further debate and demonstrated that hypoglycaemia was a controversial topic worldwide and was not just a local issue. Long before CBWC were introduced, the NNU Quality Coordinator vividly remembered having to write seven drafts of the original hypoglycaemia guideline. Anything involving BSL was challenging to implement. The UNICEF document was discussed at a perinatal meeting and became instrumental in adapting the hypoglycaemia guideline. It provided the evidence for changing the timing and number of BSL and discussed how hypoglycaemia was less critical in larger babies. This eased some of the concerns people had about increased intervention on babies. A flowchart for the management of babies with hypoglycaemia (Appendix L) was also added to the completed guideline.

The Midwifery Educator was the 'champion' that eventually saw CBWC put into action. Many acknowledged her efforts, although they did not always agree with her methods of implementation.

She did her best to bring it in because no one else had done all the ground work and good on her, because now we are streaks ahead ... That's because of her suaveness around keeping up with research ... But the way it kind of happened is, this is it and just do it. So you know it was kind of like, aye? (LMC)

The Midwifery Educator said she welcomed criticism as long as it was constructive and productive. 
When I first came and people were like we've always done it like that. I said you must never say that to me. Don't say we have always done it like that ok, that's your biggest mistake. Because I don't care what you have always done. Why are you doing it? Tell me why you are doing it and should we change it? (Midwifery Educator)

With the timely publication of the HDC findings and the UNICEF document the debate continued. These documents provided the momentum the Midwifery Educator needed to fully implement CBWC. During this time several interventions were also employed to help achieve this.

\section{2) Select, tailor, implement interventions}

Several interventions were routinely used when implementing new evidence in a guideline at the DHB. But the Midwifery Educator noted in this case the process of implementation took longer than anticipated and required repeated interventions, which is outlined in Table 4.

\section{Table 4. The implementation and intervention timeline}

\begin{tabular}{ll}
\hline Date & \multicolumn{1}{c}{ Intervention } \\
\hline 2006 & 'Management of hypoglycaemia of the newborn infant' quideline updated by \\
NNU quality coordinator. Seven drafts required as the topic initiates a lot of \\
debate and discussion. \\
-
\end{tabular}


July 2007 - Perinatal meeting presentation by Midwifery Educator on CGC and CBWC.

Sept 2007 - HDC case released to DHB CEOs. Midwifery Educator reads report and emails her comments to all relevant stakeholders. CBWC already part of a guideline but she has not been able to get the tool downloaded by the IT department.

Oct 2007 - CBWC calculator available on maternity computers.

- Amended 'Management of hypoglycaemia of the newborn infant' quideline to include CBWC to identify SGA and LGA babies.

- Ward staff meeting explaining the calculator was available and that a CBWC should be documented on the hand-over whiteboard and the infant summary sheet (see Appendix N), which was being updated.

- Midwifery Educator confirms each staff member can access the calculator and provides individual training.

Nov 2007 - HDC case presented and discussed at a perinatal meeting.

Dec 2007 - Presentation by Midwifery Educator and Clinical Director of Child Health to the clinical board on how they would prevent a similar scenario to the HDC case from happening at the DHB.

- UNICEF document 'Guidance on the development of policies and guidelines for the prevention and management of hypoglycaemia of the newborn' published.

Jan 2008 - Midwifery Educator emails stakeholders about ongoing conflict around CBWC and managing babies at risk of hypoglycaemia.

- Email communication with McCowan about some of the concerns being raised.

- Review of 'Management of hypoglycaemia of the newborn infant' quideline, to incorporate UNICEF recommendations.

Feb 2008

- $\quad$ Perinatal Meeting presentation reviewing key points from HDC case and UNICEF recommendations. Heated debate about CBWC. Draft of flow chart for 'Management of infants at risk of hypoglycaemia' presented by NNU Quality Coordinator.

- Email/letter send out to stake holders asking for further comments on flowchart.

Mar 2008 - Flowchart (see Appendix L) finalised and included in quideline. Includes changes in the timing and number of BSL and management of LGA babies.

Mid 2008 - At least two incident reports written by the Clinical Director of Child Health when the guideline was not followed correctly.

Nov 2008 - Letter sent to all LMCs by the Perinatal Committee Chairman (who was also the Clinical Director of Child Health), reminding them to complete CGC for all pregnant women.

Dec 2008

- Amended 'Management of well babies with low birth weights on the Maternity Unit' quideline to include flowchart.

Jan 2009 - Beginning date for clinical audit. Commences 13 months after the CBWC calculator was first available and 10 months after the guideline includes the flowchart. 
Each intervention allowed maternity practitioners to become more aware of the evidence and continued the debate and discussion.

Two things that this is highlighting is that even with extensive consultation there can be a problem implementing the guideline. But also the conflict actually serves to make people more aware of it. (Midwifery Educator)

\section{Main types of interventions}

\section{a) Perinatal Meetings}

These are attended by senior obstetric, senior paediatric, maternity and NNU staff, LMCs and other relevant health practitioners. It is a quality protected forum where recommendations are made to improve services. Any major changes to policies are presented here for discussion. Those implementing CBWC remember some of the discussions being quite challenging.

I remember one specific perinatal meeting where it was a quite heated discussion around it. I can't remember whether that was after the guideline was out or during the implementation period? (MM)

\section{b) Guidelines}

Any guideline changes are emailed to maternity care providers and pinned on the notice board in the maternity office. They were given one to three weeks to provide feedback on the changes. But the Midwifery Educator rarely received any comments.

I'd say look why don't you feedback and give me something and they would say oh no it's fine. If you've written it, it's fine and I'd say but that's not good enough ... You either agree or you don't and if there's things about it you are not happy with you must come back and say. Or if you know something that I don't, or whatever. (Midwifery Educator) 
Someone commented it was hard to give feedback on a guideline when you hadn't used it in clinical practice before.

Sometimes you have to actually use a protocol before you really sort of think, oh this is not there or something. (CORE)

\section{c) Flowchart}

The UNICEF document provided a sample flowchart for the management of breastfed babies at risk of hypoglycaemia. This was adapted for use at the maternity unit. Management wanted stakeholders to realise that the changes weren't just about CBWC. The changes were about improving the way they assessed babies at risk of hypoglycaemia. The flowchart encouraged this, because it required practitioners to make judgments about the care they were providing.

Nurses and midwives want a number so they can say that this baby is fine and I don't need to worry about it and this baby isn't fine and I need to worry about it. People have real barriers with making intellectual evaluations of the care they are giving. (NNU)

The timing of the first BSL was changed from being performed at one hour of age, to $>2$ hours of age, but before the second feed. Two normal consecutive BSL were required instead of three. BSL were no longer required for LGA babies if the mother had a negative diabetes screen in pregnancy. These changes made the guideline less invasive, but required core staff to be more proactive in the care they provided.

The interventions used were adapted for the local maternity service. But the CBWC calculator tool itself doesn't need to be locally adapted. It is applicable across other DHBs as it is based on the main ethnic groups in a NZ birthing population.

\section{3) Adapt knowledge to local context}

The maternity unit was part of a secondary care hospital and provided services for women covering a large urban, semi-rural and remote rural area. The DHB had a high Maori birthing population, compared to the national average of 21\% (Ministry 
of Health, 2010). Stakeholders recognised that the DHB provided services for a high risk birthing population.

We have the most socio-economically deprived area of $N Z$ and we have a very high proportion of Maori and a very high proportion of them smoke or are obese. So you know all those risk factors for IUGR babies. We do have a lot of LBW babies. We have a lot of prem babies and they are all because I believe, or most of them are due to our clientele and the smoking issue. (Midwifery Educator)

It was also recognised there was an advantage to being a smaller hospital as staffing remained relatively consistent, which made implementing change somewhat easier.

\section{4) Assess barriers to knowledge use}

Standard processes and interventions were used when introducing CBWC, such as emailing the guideline changes and perinatal meeting presentations. But practitioners were challenged more than usual by the evidence and the implementation process took longer than expected. Practitioners had a number of issues with applying the knowledge and changing the way they practiced.

\section{a) Resistance to changing practice}

Practitioners recognised that the definitions used to identify SGA and LGA babies at the maternity unit were inadequate.

This whole a birthweight of $<2500 \mathrm{~g}$ you do this, or $>4500 \mathrm{~g}$ you do this, I think it's crap. Because it just depends. Because a birthweight of $2500 \mathrm{~g}$ at 34 weeks is actually really good but a birthweight of $2500 \mathrm{~g}$ at 41 weeks is really not good. So I didn't like that arbitrary cut-off line. I thought it wasn't relevant at all. (Midwifery Educator)

In many ways people were already applying some of the underlying principles of CBWC based on their clinical experience. 
There was talk at the time that the birthweight centiles we were using were not effective working in a multi-ethnic hospital. Really and truly you were talking about little Chinese ladies to bigger Jamaican ladies. So really it just didn't make any sense. So I think we were sort of using them [CBWC] without using them ... So I think the concept was there something wasn't quite right. (CORE)

But people would dismiss the CBWC result and regress to the old definition when challenged by a baby having what they considered to be a 'normal' birthweight who was identified as 'at risk'.

There was still a little bit of resistance to the $3 \mathrm{~kg}$ baby ... and so that whole believing that those babies could still be at risk. I think took a little while. (MM)

Practitioners seemed less challenged by smaller babies being identified as SGA. They were more challenged by 'big healthy babies' having their BSLs monitored. They also found mothers were challenged by the changes as well. They questioned why their baby needed to have BSL taken when previous babies of a similar birthweight had not been monitored for hypoglycaemia. Some people also thought using CBWC was not breastfeeding friendly.

If we have got a small baby you want to be keeping that baby skin to skin and as warm as possible and we have got the core midwife there saying you need to weigh the baby and see what the CBWC is. You need to do a BSL. (LMC)

But people began to change their practice when they experienced looking after a baby that was unable to maintain its BSL, who would not have been identified previously.

Unless you have actually had them, you don't really get it. I think, because you just think oh for goodness sake they're a normal size baby blah, blah. But if you've been caught out I think that's when it really rams home to you, whereas I haven't. So I'm probably a bit more blasé. But if you have then it makes you think sh**. (LMC) 
Eventually calculating a CBWC became a part of the culture of the maternity unit.

It was incremental probably over a year ... Now it's just a routine thing. (Midwifery Educator)

The majority of stakeholders now agree that CBWC are valuable. Even those who opposed using them in the beginning have accepted calculating CBWC as good practice.

\section{b) Using the CBWC calculator}

CBWC were part of the guideline before it was accessible on the maternity unit computers. But the delay in downloading of the calculator was rectified immediately once the issue was brought to the attention of the CEO. Use of the calculator relies on the accurate collection and recording of data and accurate data entry. Previously most LMCs were not routinely collecting pre-pregnancy weight and height, which are required to calculate a CBWC.

The height and weight and BMI, again that's another change that's incremental. Now $99 \%$ of women have them done ... That took ages to get people to understand that ... If you've got somebody with a BMI of over 30 you've actually got more risk factors in the pregnancy anyway. (Midwifery Educator)

But there was variation in how this data was collected which caused some to question the accuracy of the CBWC result.

Sometimes they don't know their pre-pregnancy weight. (LMC)

I just put the weight and say how many weeks they are. (LMC)

I've had people turn up at 32 weeks and it's not relevant. (LMC)

When this information was missing sometimes the core staff would estimate a value or ask the woman what it was postnatally. 
The other thing I've done is guesstimated you know. You can put in she's about this tall and about this weight and this is how much it would be and you think oh that's ok. (Midwifery Educator)

Everyone agreed the calculator was easy to use. But because the data was entered manually people also commented that it was easy to make a mistake or manipulate results. Both LMCs and core staff talked about seeing others 'fix' the CBWC result by changing a data value so a baby was no longer identified as SGA or LGA.

Some of them are just like, nah I'm sure her heights this, oh no I think it's, you know like sometimes people almost want to try and fix the results ... they try to recheck and dates might be the one thing they try to, because if the baby can be slightly older or under it can make one point of difference. (LMC)

But some LMCs and core staff disagreed with this practice.

Sometimes the borderline babies with their weight are the ones that are crashing. (LMC)

\section{c) Increased intervention}

Using CBWC resulted in an increased number of babies identified as SGA or LGA, which meant more babies received heel pricks. People weren't concerned about the increase in their workload but were concerned about the increased invasiveness of the procedure.

When it involves an invasive heel prick ... that's where people are reluctant to change their practice and I think that's probably around if you don't believe it yourself trying to convince the parents that us you know doing a heel prick on your baby is a good thing. People struggled with that. (MM) 
The guideline was modified accordingly to alleviate peoples' concerns.

We tweaked it at the end so the guideline became less invasive. I think that's probably what helped settle it. It was around the timing of BSL and around how many ... I think that probably helped people. (MM)

But despite this change people admitted performing more BSL than required because they were used to the old protocol. But all agreed that performing a heel prick was better than a baby developing brain damage and the way this information was shared with mothers and their families was important.

\section{d) Following the guideline correctly}

Most of the resistance came after CBWC were included in the appropriate guidelines and people had forgotten about the education and consultation process that had occurred months prior.

Often the people say they are fine with it and then the resistance comes after the guideline is signed off and the writers of the guidelines want feedback before it is put out. (Midwifery Educator)

There was confusion over what people believed the guideline did and didn't say. People were unaware that the baby's temperature, respirations and heart rate were also meant to be recorded. They were unaware that population BWC charts (Appendix O) were to be used when data was missing and a CBWC couldn't be calculated. There was confusion around monitoring LGA babies. Many did not realise that BSL were not required if the mother had a negative diabetes screen in pregnancy. Others questioned the accuracy of the polycose screening test and monitored the baby regardless of the polycose result.

They have just changed the polycose thing so it's not really applicable to pregnancy. So they would have been raised on the old one, but because they have now moved the boundaries up with the new polycose it's showing them as normal and it's like, well which one do we go by? Cause the new one isn't really applicable in pregnancy. (CORE) 
Incident reports were written by the Clinical Director of Child Health, when the guideline was not followed.

There was a macrosomic baby that was on the $97^{\text {th }}$ centile and the Paed ended up writing an incident report about that one because the protocol wasn't followed correctly. (LMC)

Some women also refused to have their babies monitored.

Actually women still have the right to say no ... and some women did, even after being informed they said no. (LMC)

But midwives were cautioned about the information they were sharing with women.

If you tell the mums what our guideline is and then they decline, then that's ok. It's you as a professional making that decision to do it on behalf of them, that's when it's not I don't believe ok ... You're a professional but actually it's about the parents making the decision, not you ... It's around if you have that discussion with her then you must document it. But if you didn't have the discussion stop and think about is it your perception or is it the mother's? So get that clear in your head. (MM)

There were also unwritten rules around how the CBWC was recorded.

[It's written in] three places. One's on the board, one's on the neonate infant newborn summary and one's in the register. (Midwifery Educator)

Also missing from the guideline was instructions on whose role it was to calculate the CBWC.

\section{e) Primary or secondary care responsibility?}

There were differing views on whose responsibility it was to calculate the CBWC. One issue was that LMCs were not given computer logins so they couldn't access the maternity unit computers. But the calculator was usually available on any computer desktop which had been logged on. 
I thought it was the job of the core midwife on handover to do the $C B W C$ but somebody said to me recently, oh no it says in the guideline it's the LMCs responsibility, and I thought oh? I can't remember putting that down. I thought it was on handover, you know once the woman is handed over postnatally you need to do a $C B W C$. (Midwifery Educator)

But some LMCs believed it was their responsibility and would calculate it themselves or ask someone to do it for them.

I think I'd like to have it done before you leave though so you know and especially you can get some that kind of surprise you and have a low CBWC and you think whoa I wouldn't have picked that one and so that baby is going to need obs. So if you think about it, if you are leaving after the two hours that baby might be due another feed anyway or might be just about to go on the breast and so you are wanting to get that BSL done before that. So I think it is a good idea to have it done before you leave. (LMC)

The core staff believed the calculation should be part of the care provided by the LMC.

That's part of the first assessment ... If they can do the baby check, the full baby check, then why can't they just quickly sit down for two minutes and do a CBWC. (CORE)

But the core staff felt that calculating the CBWC was largely left for them to complete. Even though they felt it was inappropriate that they were the ones having to inform the women of the result.

They are the ones [providing] primary care so if anything has got to change about that like a baby needing obs and BSL, then they should be the ones that inform the women of it. Rather than a face that they have never met before coming in and going actually I've got to prick your baby's heel every 3 hours. (CORE) 
The core staff admitted that sometimes opportunities were missed, especially when the ward was busy.

A couple of times it's been a good few hours, you know six hours afterwards and you are like crap, oh what's that CBWC and you work it out and it's low and you are like oh no. (CORE)

But if LMCs and core staff worked together this would not be an issue.

When it's quiet we can do it and get on with it. But when we are running around like a lunatic and there's only one midwife on shift ... it would be helpful in that situation that when we are busy that they could just do it [the LMC] so that we wouldn't have to worry about babies slipping through the net. (CORE)

Most people agreed that regardless of who calculated it, the LMC should be aware of the CBWC result before handing over care of the baby to core staff.

\section{Part B: Measuring the extent of implementation, the story continues ...}

The red shaded parts of the action cycle (Figure 5, p. 42) were about measuring the extent to which the CBWC had been implemented as intended and what outcomes had resulted from it. No formal monitoring of outcomes had been done by the DHB itself. But people believed after the initial challenges, that calculating CBWC had become part of the culture of the maternity unit. They also believed using CBWC was making a difference in identifying babies at risk that previously would have been missed. A clinical audit was conducted as part of this research, to measure the extent of implementation and evaluate outcomes. Qualitative findings were also used to continue telling the story and validate the quantitative outcomes of the audit.

\section{5) Monitor knowledge use}

Nothing had been done by the DHB to monitor knowledge use, but stakeholders felt the knowledge of CBWC had initiated a proactive individualised management approach to neonatal hypoglycaemia. Practitioners also began to notice how CBWC 
fit into the bigger picture of risk assessment and started making appropriate connections.

\section{a) Proactive management}

Using CBWC had resulted in a general increased awareness around identifying neonatal risk of hypoglycaemia. The UNICEF document was instrumental in developing a flowchart which promoted a proactive approach to the management of hypoglycaemia.

It's actually promoting looking at that child in totality and not normalising the abnormal but not abnormalising the normal either. So if you've got a child that's got a low $C B W C$, yes you are going to have to be proactive about the thermal management of that baby, feeding, about a multiplicity of things. You might have to be really careful about early discharge ... So I think it was making that intellectual shift to actually really looking at the baby instead of just wanting a number and yes that BSL is ok and I can ignore it. So I think it's an intellectual process and I think that has changed. (NNU)

As a result, it appeared the number of babies admitted to the neonatal unit with hypoglycaemia had decreased.

I haven't seen one of those ... for a year or so. Where they come from the ward, they are really really sleepy looking, absolutely dreadful, unrecordable BSL and then you send it off to the lab and get a 0.6 or something. We are not getting those now because I think we are more alert to those. (NNU)

Staff were no longer waiting for the non-feeding baby to show signs of hypoglycaemia before deciding to intervene in some way.

\section{b) Individualising care}

Stakeholders felt that using CBWC was better than the old birthweight cut-off points as each baby was now considered individually. 
Now it's individualised, you are going by what is normal for that mum based upon her ethnicity, her parity, her height and weight, because we never put those factors in [before]. (LMC)

Using CBWC picked up babies that were previously missed and it seemed to be an effective tool.

There was one that was a 3 on the centile and it was admitted eventually for a week ... It was $3.2 \mathrm{~kg}$ but on the $3^{\text {rd }}$ centile. (LMC)

The CBWC result become more than just a number and practitioners started to make connections with how it fit into the bigger picture of 'risk' for the mother and baby.

\section{c) Making connections}

People saw the value of CBWC in being about more than just the risk of hypoglycaemia.

Really it is a screening tool and if anything it has helped me identify risk, risk behaviour and almost predicting outcomes. (LMC)

A link was noticed with the importance of effective breastfeeding.

I think it's made people more aware ... in general of the risk factors around babies feeding patterns and stuff and around the importance of actually seeing, making sure a baby has fed well. Rather than just taking the mother's word for it. (MM)

It also increased awareness of how smoking in pregnancy was related to birthweight.

Getting women to reduce smoking is the biggest thing we can do to reduce perinatal mortality ... and CBWC is something that makes up the whole picture. (Midwifery Educator)

Many of the LMCs said since CBWC were implemented, they now routinely screened women for diabetes. 
I think that because we've got the growth charts in pregnancy, the GROW package, we are doing a lot to try and educate women around diet ... So I haven't seen so many big babies over the 95th centile and since we have been screening all women for diabetes they are kind of picked up. (LMC)

Connections were also made with a woman's BMI and risk.

I just had someone recently who is pregnant again and I did her's and she's obese and her baby came out SGA. Her previous baby and I thought it wasn't a huge baby, but a normal sized baby and quite interesting because of her weight it made her baby SGA. (LMC)

People saw how CBWC linked with CGC charts completed in pregnancy. When a baby was born with a low CBWC people started asking if the result had been unexpected and whether this was picked up antenatally.

This baby had a CBWC of zero. Did you not pick it up antenatally? What happened? (LMC)

An LMC talked about one woman and how the CBWC result highlighted the other risk factors of her pregnancy.

Smoking in pregnancy, alcohol involved ... formula feeding ... she kind of likes to co-sleep. So huge risk factors around that baby being a SIDS risk ... This baby was $2690 \mathrm{~g}$ and see it was 42 weeks gestation and because of her size and her parity and stuff like that the baby wasn't $2500 \mathrm{~g}$ but it was a zero CBWC. So it's been good in picking up babies like that. (LMC)

After these experiences people became more vigilant in calculating the CBWC because they felt it identified babies who were previously missed. The CBWC was seen as an effective screening tool and more than just a number. 


\section{6) Evaluate outcomes}

Stakeholders believed that the guideline was being followed correctly and that using CBWC was making a difference. But no one had evaluated whether this was indeed true.

Because nobody's actually as far as I understand now looked at the research ... after implementing it, to justify your actions, I suppose to see actually now yes it has improved outcomes for babies. (MM)

Therefore, a clinical audit was performed to try to answer these questions and evaluate outcomes.

\section{Results of the clinical audit}

There were 303 births that fit the final inclusion criteria (Table 5). The audit covered two three month periods, beginning and ending one year apart. Births included in the audit were: $\geq 37$ weeks gestation, no stillbirths, no congenital abnormalities, no multiple births and no BBA's (born before arrival). Data provided by the Maternity Unit Manager indicated the total number of births in 2009 was 701 . Several outcomes were evaluated relating to following processes and which babies were identified by CBWC.

Table 5. Number of births included in the audit

\begin{tabular}{lccc}
\hline & $\begin{array}{c}\text { Jan 2009 } \\
\text { Feb 2009 } \\
\text { Mar 2009 }\end{array}$ & $\begin{array}{l}\text { Nov 2009 } \\
\text { Dec 2009 } \\
\text { Jan 2010 }\end{array}$ & TOTAL \\
\hline $\begin{array}{l}\text { Number of births fitting } \\
\text { the inclusion criteria }\end{array}$ & 141 & 162 & 303 \\
\hline
\end{tabular}

\section{a) Were processes followed correctly?}

Each CBWC was supposed to be recorded on the infant summary sheet (Appendix $\mathrm{N})$ and in the birthing register. According to the guideline, if a CBWC could not be calculated due to missing data, then sex-adjusted population birthweight centile charts (Appendix O) were to be used. Whether this process was followed or not was audited (Table 6). 
The CBWC was recorded on the infant summary sheet and/or the birthing register $76.2 \%$ of the time. This improved over the audit period from $71.6 \% \rightarrow 80.2 \%$. Extrapolated for all births in 2009, potentially the CBWC would not have been recorded 167 times $(23.8 \%)$.

The maternal details were missing and no attempt was made to record a CBWC result, $3.6 \%$ of the time. This improved over the audit period as the percentage decreased from $5 \% \rightarrow 2.5 \%$. For these 11 cases, the population BWC charts should have been used. The maternal results were missing 22 times (7.3\% (22/303), but for half of these a CBWC result was recorded. So it was unknown how this result was calculated.

Table 6. Number of times the CBWC was calculated and recorded

\begin{tabular}{lccc}
\hline & $\begin{array}{c}\text { Jan 2009 } \\
\text { Feb 2009 } \\
\text { Mar 2009 }\end{array}$ & $\begin{array}{c}\text { Nov 2009 } \\
\text { Dec 2009 } \\
\text { Jan 2010 }\end{array}$ & TOTAL \% \\
\hline $\begin{array}{l}\text { Number of times the CBWC was } \\
\text { recorded (on the infant summary } \\
\text { sheet and/or the register) }\end{array}$ & $71.6 \%(101 / 141)$ & $80.2 \%(130 / 162)$ & $76.2 \%(231 / 303)$ \\
$\begin{array}{l}\text { CBWC recorded on infant summary } \\
\text { sheet }\end{array}$ & $66 \%(93 / 141)$ & $74.7 \%(121 / 162)$. & $70.6 \%(214 / 303)$ \\
$\begin{array}{l}\text { CBWC recorded in birthing register } \\
\text { Number of times maternal details }\end{array}$ & $26.2 \%(37 / 141)$ & $50 \%(81 / 162)$ & $38.9 \%(118 / 303)$ \\
$\begin{array}{l}\text { were missing and no attempt was } \\
\text { made to record a CBWC }\end{array}$ & $5 \%(7 / 141)$ & $2.5 \%(4 / 162)$ & $3.6 \%(11 / 303)$ \\
\hline
\end{tabular}

In the 11 cases where no CBWC was recorded, I used the sex-adjusted population birthweight centile charts as the guideline indicated (Appendix N). For all 11 cases the population BWC was within the normal range (between the $10^{\text {th }}$ and $95^{\text {th }}$ centile) selected by the DHB.

Almost a quarter of the time there was no evidence of a CBWC having been recorded, but this didn't necessarily mean that it wasn't calculated. It is possible that 
the result was written on the handover whiteboard and was calculated appropriately. But this result is erased at the time of discharge. When the CBWC is not recorded in the notes or birthing register, it gives the impression that it was not done.

We write them in red up on the board, so it should be pretty obvious now whether it's been done or not ... Although I would have to say that probably they never or very rarely get filled on the green form [infant summary sheet] ... The receptionist first thing in the morning when they come in they fill in the register. (MM)

When staff were asked if they had ever used the population BWC charts the unanimous response was:

What? (CORE)

People didn't realise that using them was part of the guideline and were unfamiliar with what the population BWC charts looked like and where they were located.

The CBWC recorded and the CBWC calculated by myself were compared (Table 7). I was only able to compare a CBWC for 220 births. $27.4 \%$ of the time either some of the required data was missing, or a CBWC had not been recorded. From these 220 births, $62.7 \%$ of the time I calculated the same CBWC as the one that was recorded. This improved over the audit period from $52 \% \rightarrow 71.3 \%$. Extrapolated for all births in 2009, this means that potentially two people using the same data could calculate and record a different CBWC result for 261 births (37.3\%).

Calculating a different result $37.3 \%$ of the time appears high. But in reality this only resulted in five instances where the clinical management of the baby would have changed. For example, if I calculated a CBWC of 61 but 63 had been recorded, the baby would have still had a CBWC in the normal range. Any baby with a CBWC $<10$ or $>95$ (if the mother did not have a negative diabetes screen in pregnancy) was required to have BSL monitoring. Of these five cases, there appeared to be evidence of some of these results being purposely changed. 
- For three cases I calculated a CBWC of 9, whereas a CBWC of 10 had been recorded. The birthweights of these babies were $3180 \mathrm{~g}, 3300 \mathrm{~g}$ and $3100 \mathrm{~g}$.

- In one case I calculated a CBWC of 96, whereas a CBWC of 95 had been recorded. The birthweight of this baby was $4220 \mathrm{~g}$.

- In one case I calculated a CBWC of 94 and 97 had been recorded. The birthweight of this baby was $4540 \mathrm{~g}$. It appeared a calculation error had been made.

In the first four cases $(1.8 \%(4 / 220))$ discussed above, the results appear to have been purposely manipulated.

It was not surprising to find evidence of results being manipulated as this practice had been disclosed in the interviews and focus groups. But nobody admitted doing it themselves.

Because I suppose of the different kind of perceptions staff have of the CBWC and doing BSLs they will say, oh look that baby looks perfectly alright for goodness sake it's just under. Lets say if we made it umm that she weighed this, you know take a couple kilos off or make her a cm taller, then the baby is in the normal range and they say now we don't have to prick it's foot. (LMC)

This meant that four babies with what appeared to be 'normal' birthweights, were not identified as SGA or LGA. Extrapolated for all births in 2009, potentially the CBWC results would be manipulated for 13 births. 


\begin{tabular}{lccc}
\hline & $\begin{array}{l}\text { Jan 2009 } \\
\text { Feb 2009 } \\
\text { Mar 2009 }\end{array}$ & $\begin{array}{l}\text { Nov 2009 } \\
\text { Dec 2009 } \\
\text { Jan 2010 }\end{array}$ & TOTAL \% \\
\hline $\begin{array}{l}\text { Number of births where I was able to } \\
\text { calculate a CBWC and compare it } \\
\text { with a recorded CBWC }\end{array}$ & $69.5 \%(98 / 141)$ & $75.3 \%(122 / 162)$ & $72.6 \%(220 / 303)$ \\
$\begin{array}{l}\text { Number of times where the CBWC I } \\
\text { calculated was the same as the }\end{array}$ & $52 \%(51 / 98)$ & $71.3 \%(87 / 122)$ & $62.7 \%(138 / 220)$ \\
$\begin{array}{l}\text { recorded CBWC } \\
\begin{array}{l}\text { Number of times when the difference } \\
\text { between the CBWC I calculated and } \\
\text { the CBWC that was recorded could } \\
\text { have been of clinical significance }\end{array}\end{array}$ & $2 \%(2 / 98)$ & $2.5 \%(3 / 122)$ & $2.3 \%(5 / 220)$ \\
\hline
\end{tabular}

\section{b) The number of babies identified as SGA or LGA}

For the remaining outcomes, the audit sample will remain at 303 births. A CBWC was calculated and recorded for 292 births, either by myself or the staff/LMCs. There were only 11 babies where no CBWC was able to be calculated or recorded. But the population BWC for all 11 babies were within the normal range. Therefore the audit sample size remained at 303. The babies identified as SGA or LGA were based on my calculations. I believed my calculations were the most accurate, as I had re-checked my results multiple times.

The number of babies identified as SGA or LGA using CBWC versus the cut-off weights of $<2500 \mathrm{~g}$ and $>4500 \mathrm{~g}$, were compared (Table 8). It shows that when using CBWC a larger number of babies were identified as SGA or LGA.

There were $10.9 \%$ of babies identified as SGA using CBWC, compared with $3.6 \%$ of babies using the $<2500 \mathrm{~g}$ cutoff. There were $4.6 \%$ of babies identified as LGA using CBWC, compared with $3 \%$ of babies using the $>4500 \mathrm{~g}$ cutoff. Twenty-two (7.3\%) more babies were identified as SGA using CBWC. Extrapolated for all births in 2009, potentially 51 more babies would have been identified as SGA if CBWC were used. Five (1.6\%) more babies were identified as LGA using CBWC. 
Extrapolated for all births in 2009, potentially 12 more babies would have been identified as LGA if CBWC were used.

Table 8. Numbers of SGA and LGA babies identified by CBWC vs cut-off weights

\begin{tabular}{|c|c|c|c|}
\hline & $\begin{array}{l}\text { Jan } 2009 \\
\text { Feb } 2009 \\
\text { Mar } 2009\end{array}$ & $\begin{array}{l}\text { Nov } 2009 \\
\text { Dec } 2009 \\
\text { Jan } 2010\end{array}$ & TOTAL \% \\
\hline SGA babies by CBWC & $9.9 \%(14 / 141)$ & $12 \%(19 / 162)$ & $10.9 \%(33 / 303)$ \\
\hline SGA babies by $<2500 \mathrm{~g}$ & $2.8 \%(4 / 141)$ & $4.3 \%(7 / 162)$ & $3.6 \%(11 / 303)$ \\
\hline $\begin{array}{l}\text { Additional babies identified as SGA } \\
\text { by CBWC vs }<2500 \mathrm{~g}\end{array}$ & $7.1 \%(10 / 141)$ & $7.4 \%(12 / 162)$ & $7.3 \%(22 / 303)$ \\
\hline LGA babies by CBWC & $3.5 \%(5 / 141)$ & $5.6 \%(9 / 162)$ & $4.6 \%(14 / 303)$ \\
\hline LGA babies by $>4500 \mathrm{~g}$ & $2.1 \%(3 / 141)$ & $3.7 \%(6 / 162)$ & $3.0 \%(9 / 303)$ \\
\hline $\begin{array}{l}\text { Additional babies identified as LGA } \\
\text { by CBWC vs }>4500 \mathrm{~g}\end{array}$ & $1.4 \%(2 / 141)$ & $1.9 \%(3 / 162)$ & $1.6 \%(5 / 303)$ \\
\hline
\end{tabular}

The number of babies who were identified as SGA by CBWC only (22), the number of SGA babies identified by $<2500 \mathrm{~g}$ only ( 0 ) and the number of babies identified by both methods (11), are shown in Figure 6. All babies $<2500 \mathrm{~g}$ were still identified as SGA when using CBWC. (Note: In Figure 6 the size of the circles and overlap is not drawn to scale).

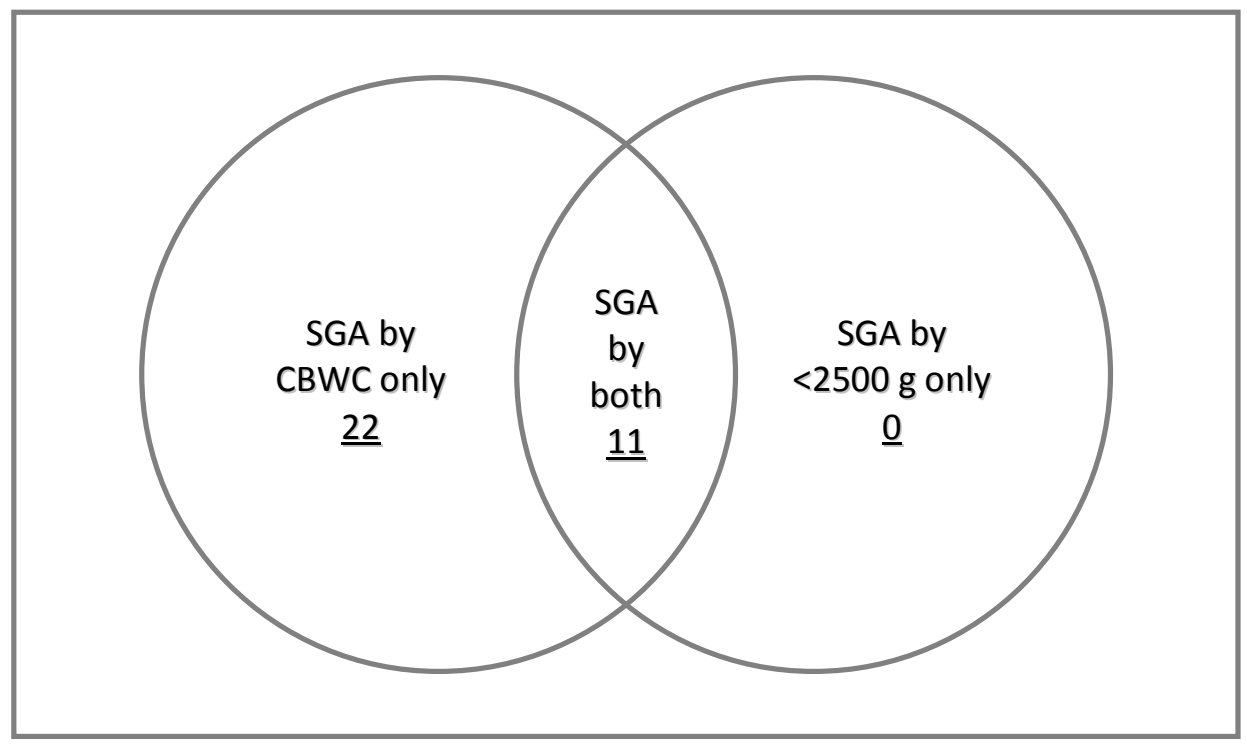

Figure 6. The classification of SGA babies by CBWC vs $<2500 \mathrm{~g}$ 
The data for the 22 babies that were identified as SGA by CBWC only is shown in Table 9. A few notes about each of these babies are provided. Among this list are examples of babies who potentially could have become unwell if their risk of hypoglycaemia was not recognised early. For example, baby \#20 was admitted to the NNU after recording a low BSL before and after its first breastfeed. It continued to have low BSL results despite receiving intravenous (IV) Glucose and nasogastric tube (NGT) feeds. The mother was Maori, obese and having her second baby. In other examples babies required intervention in the form of breastfeeding assistance, hand expressing and expressed breast milk (EBM) and/or formula top ups to correct low BSLs.

While more babies were identified, this doesn't demonstrate whether fewer babies experienced morbidity or mortality relating to hypoglycaemia. It also showed that even though CBWC were implemented, this doesn't guarantee babies aren't missed. For example, baby \#15 was discharged one and a half hours after birth and no early discharge form was completed. The baby's birthweight was 3500g and no CBWC was recorded. I calculated the $\mathrm{CBWC}$ as 9. The clinical notes explained the baby had measured small in pregnancy until 37 weeks. At 38 weeks there was a marked growth in fundal height. At 39 weeks the fundal height again plotted small for gestation. This was an example of a CBWC not being recorded and a SGA baby being discharged early. In another example, baby \#16 didn't have a CBWC calculated until it was eight and a half hours old. This baby was eventually admitted to the NNU with borderline BSLs and poor feeding. These were both examples of missed opportunities where a baby potentially could have become very unwell.

The average BMI for the mothers of these babies was $28.1 \mathrm{~kg} / \mathrm{m}^{2}$ (range: $19-42.2$ $\mathrm{kg} / \mathrm{m}^{2}$ ). In $\mathrm{NZ}$ a BMI of $25-29.9 \mathrm{~kg} / \mathrm{m}^{2}$ is considered overweight and a BMI $\geq 30$ $\mathrm{kg} / \mathrm{m}^{2}$ is considered obese (C. T. R. U. Ministry of Health, 2009). The average birthweight for these babies was $2915 \mathrm{~g}$ (range: 2500 - $3500 \mathrm{~g}$ ), which is well above the previous cut-off point of $2500 \mathrm{~g}$. 
Table 9. 22 babies identified as SGA using CBWC who would not have been previously identified

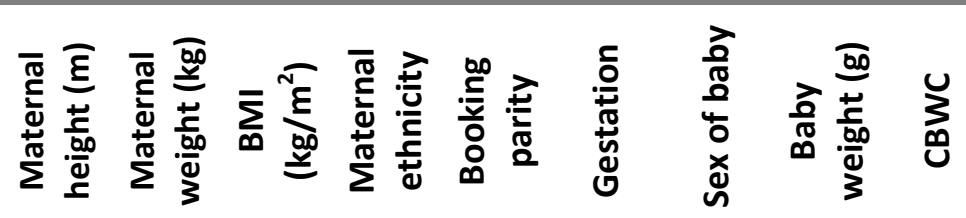

Notes

\begin{tabular}{|c|c|c|c|c|c|c|c|c|c|c|}
\hline 1 & 1.79 & 74 & 23.1 & M & 0 & $40+4$ & M & 3180 & 9 & Normal BSLs \\
\hline 2 & 1.72 & 83 & 28.1 & M & 0 & $39+6$ & $\mathrm{~F}$ & 2920 & 7 & Guideline not followed properly \\
\hline 3 & 1.67 & 80 & 28.7 & M & 4 & $41+3$ & M & 3120 & 2 & Deceased one month after birth, no cause recorded \\
\hline 4 & 1.70 & 55 & 19.0 & $\mathrm{E}$ & 0 & $40+2$ & $\mathrm{~F}$ & 2920 & 8 & Written in mothers notes for no BSL, no reason given \\
\hline 5 & 1.76 & 65 & 21.0 & $\mathrm{E}$ & 0 & $40+5$ & $\mathrm{~F}$ & 3000 & 6 & Didn't feed well, hand expressing, nipple shield, 3 normal BSL \\
\hline 6 & 1.58 & 95.4 & 38.2 & M & 5 & $39+6$ & $\mathrm{~F}$ & 2780 & 4 & Mother was gestational diabetic \\
\hline 7 & 1.57 & 104 & 42.2 & M & 1 & $39+2$ & M & 2660 & 3 & Required formula after low BSL, then normal BSL \\
\hline 8 & 1.72 & 99 & 33.5 & M & 0 & $40+6$ & $\mathrm{~F}$ & 2940 & 4 & $\begin{array}{l}\text { Sleepy baby, BF and EBM given, multiple BSL taken, one result } 2.3 \\
\text { all others normal }\end{array}$ \\
\hline 9 & 1.70 & 110 & 38.1 & M & 0 & $39+1$ & M & 2915 & 7 & $\begin{array}{l}\text { Apgar: } 7,7 \text {, lots of BF assistance, given formula and EBM top ups, } \\
\text { one BSL } 2.5 \text {, all others normal but }<3 \text {, admitted Day } 6 \text { for jaundice }\end{array}$ \\
\hline 10 & 1.64 & 56 & 20.8 & M & 0 & $40+4$ & $M$ & 2905 & 8 & Apgar: 5,8 , first BSL 1.7 , then normal \\
\hline 11 & 1.65 & 65 & 23.9 & $\mathrm{E}$ & 0 & $42+1$ & M & 3300 & 9 & $\begin{array}{l}\text { Apgar: } 7,9 \text {, cold, large capput, jaundiced but normal result, BF } \\
\text { assistance required }\end{array}$ \\
\hline 12 & 1.55 & 65 & 27.1 & M & 0 & $41+1$ & $\mathrm{~F}$ & 2720 & 3 & 2 normal BSL \\
\hline 13 & 1.67 & 63 & 22.7 & $M$ & 0 & $40+2$ & $\mathrm{~F}$ & 2860 & 8 & Multiple BSL taken as two results $<2.6$ \\
\hline 14 & 1.63 & 60 & 22.6 & $\mathrm{E}$ & 2 & 41 & $\mathrm{~F}$ & 2960 & 5 & BF well, normal BSL \\
\hline 15 & 1.80 & 92 & 28.4 & $\mathrm{E}$ & 1 & 41 & M & 3500 & 9 & Discharged at 1.5 hours old, no CBWC recorded \\
\hline 16 & 1.68 & 83 & 29.4 & $\mathrm{E}$ & 2 & $40+6$ & $\mathrm{~F}$ & 2980 & 3 & $\begin{array}{l}\text { CBWC not done until } 8.5 \text { hours old, multiple BSL between } 2.2-2.8 \\
\text { with most }<2.6 \text {, to NNU for poor feeding }\end{array}$ \\
\hline 17 & 1.65 & 70 & 25.7 & $\mathrm{E}$ & 5 & 42 & $\mathrm{~F}$ & 2680 & 0 & Formula fed, chose not to BF \\
\hline 18 & 1.65 & 91 & 33.4 & M & 3 & 40 & $\mathrm{~F}$ & 2680 & 2 & 4 normal BSL \\
\hline 19 & $?$ & 54 & $?$ & $M$ & 0 & $40+1$ & $M$ & 2500 & 0 & Normal BSL \\
\hline 20 & 1.56 & 73 & 30.2 & $M$ & 1 & $41+1$ & $\mathrm{~F}$ & 2860 & 3 & $\begin{array}{l}\text { Admitted to NNU, low BSL before and after the first breastfeed, } \\
\text { BSLs of } 1.5,1.6,1.3 \text {, borderline BSLs continued despite IV Glucose } \\
\text { and NGT feeds }\end{array}$ \\
\hline 21 & 1.71 & 65 & 22.2 & $M$ & 4 & $38+1$ & $M$ & 2640 & 4 & Took time to establish lactation, formula top ups after $1.4 \mathrm{BSL}$ \\
\hline 22 & 1.70 & 92.6 & 32.0 & M & 0 & $40+5$ & $\mathrm{~F}$ & 3100 & 9 & $\begin{array}{l}\text { No BSL done, lots of BF assistance, unsettled baby, maternal } \\
\text { request for formula }\end{array}$ \\
\hline Mean & 1.67 & 77 & 28.1 & & 1.3 & $40+4$ & & 2915 & 5 & \\
\hline
\end{tabular}


The number of babies identified as LGA by CBWC only (8), the number of babies identified by $>4500 \mathrm{~g}$ only (3) and the number of babies identified by both methods (6) are shown in Figure 7. Not all babies $>4500 \mathrm{~g}$ were identified as LGA when using CBWC. (Note: In Figure 7 the size of the circles and overlap is not drawn to scale).

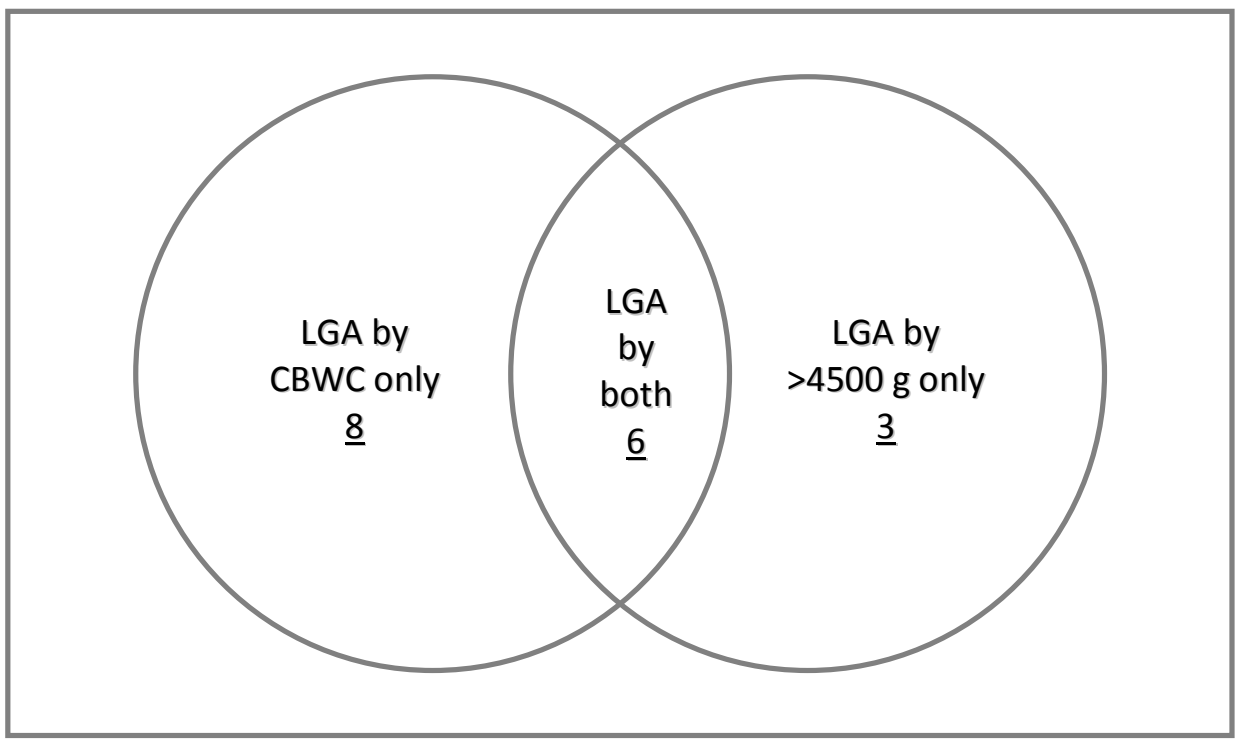

Figure 7. The classification of LGA babies by CBWC vs $>4500 \mathrm{~g}$

The data for the 8 babies that were identified as LGA by CBWC only are listed in Table 10. There may appear to be a discrepancy with Table 8 (p.83) which showed five more babies were identified as LGA using CBWC vs $4500 \mathrm{~g}$. The difference of three babies is explained by the fact that not all babies who were $>4500 \mathrm{~g}$ were identified as LGA when CBWC were used. A few notes about each of these babies are provided. From this list there is one example of a baby who could have potentially become unwell if their risk of hypoglycaemia was not recognised early. Baby \#1 was admitted to the NNU with low BSL that didn't improve with formula top ups and the baby's birthweight was $4280 \mathrm{~g}$. There were also some examples of BSL monitoring being performed incorrectly as the mother had a normal polycose. But in some of these examples the baby did have low BSL. For example, baby \#7 and baby \#8 had low BSL but their mothers had a normal polycose results. This sample is small so no conclusive results could be drawn from these examples. 
While more babies were identified as LGA, this doesn't demonstrate whether less babies experienced morbidity or mortality relating to hypoglycaemia since using CBWC.

The average BMI for the mothers of these babies was $37.3 \mathrm{~kg} / \mathrm{m}^{2}$ (range: 23 - 59.7 $\mathrm{kg} / \mathrm{m}^{2}$ ). In $\mathrm{NZ}$ a BMI of $35-39.9 \mathrm{~kg} / \mathrm{m}^{2}$ is considered to be moderately obese (C. T. R. U. Ministry of Health, 2009). The average birthweight for these babies was 4123 $\mathrm{g}$ (range: $3800-4440 \mathrm{~g}$ ), which is well below the previous cut-off point of $4500 \mathrm{~g}$. 
Table 10. 8 babies identified as LGA using CBWC who would not have been previously identified

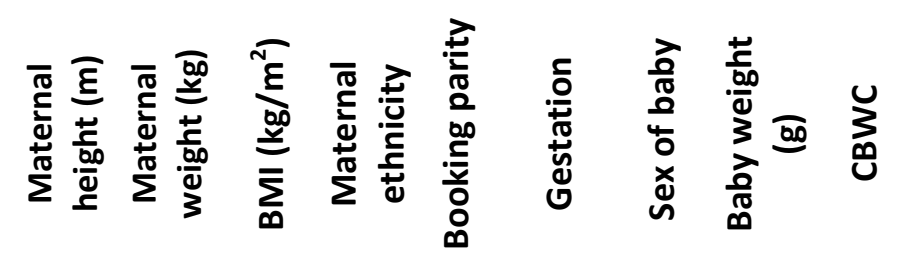

\begin{tabular}{|c|c|c|c|c|c|c|c|c|c|c|}
\hline & & & & & & & & & & \\
\hline 1 & 1.7 & 150 & 51.9 & $\mathrm{M}$ & 1 & $37+5$ & M & 4280 & 99 & $\begin{array}{l}\text { Low BSL 1.9, 2.1, didn't improve with formula top ups, admitted to } \\
\text { NNU for BSL, top ups, jaundice, no maternal polycose }\end{array}$ \\
\hline 2 & 1.64 & 109 & 40.5 & $M$ & 3 & $38+5$ & M & 4220 & 96 & BSL not required \\
\hline 3 & 1.4 & 65 & 33.2 & $M$ & 1 & $38+6$ & M & 3800 & 97 & $\begin{array}{l}\text { Had one BSL 2.1, had to be woken for feeds, took time to establish } \\
\text { BF, extra normal BSL done }\end{array}$ \\
\hline 4 & 1.52 & 138 & 59.7 & $E$ & 1 & 40 & $M$ & 4420 & 97 & Normal maternal polycose \\
\hline 5 & 1.6 & 59 & 23.0 & $M$ & 2 & 39 & $\mathrm{~F}$ & 3860 & 96 & Normal BSL \\
\hline 6 & 1.72 & 84.5 & 28.6 & $\mathrm{E}$ & 2 & $37+1$ & M & 3965 & 96 & $\begin{array}{l}\text { Admitted to NNU from CS, CPAP, No CBWC recorded, BSL in NNU } \\
\text { and immunoglobulin transfusion }\end{array}$ \\
\hline 7 & 1.58 & 69.9 & 28.0 & I & 1 & $38+6$ & $\mathrm{~F}$ & 4000 & 99 & $\begin{array}{l}\text { Six BSL done, two were } 2.2,2.5 \text {, but were stopped when it was } \\
\text { noted the maternal polycose was normal }\end{array}$ \\
\hline 8 & 1.68 & 94 & 33.3 & $E$ & 1 & $39+3$ & $\mathrm{~F}$ & 4440 & 98 & $\begin{array}{l}\text { Had low BSL } 2.5,1.5 \text {, several borderline results, not admitted to } \\
\text { NNU, BF well, normal maternal polycose }\end{array}$ \\
\hline Mean & 1.61 & 96.2 & 37.3 & & 2 & $38+5$ & & 4123 & 97 & \\
\hline
\end{tabular}




\section{c) Was the guideline followed correctly?}

The audit investigated whether BSL were initiated for babies that were identified as SGA (Table 11). There were 29 babies recorded as being SGA. In all but one instance BSL were initiated. In this case the baby had a CBWC of 8 and a birthweight of $2920 \mathrm{~g}$. The CBWC had been recorded and underlined on the appropriate feed chart, but only temperature, respirations and heart rate observations were taken. 'No BSL taking' had been written in the maternal notes, with no further explanation given. This was possibly an example of a mother deciding against BSL monitoring, but observations were still performed to watch for symptoms of hypoglycaemia.

Table 11. Babies recorded as SGA and whether BSL were initiated according to the guideline

\begin{tabular}{lccc}
\hline \multicolumn{1}{c}{ the guideline } & Jan 2009 & Nov 2009 & \\
& $\begin{array}{l}\text { Feb 2009 } \\
\text { Mar 2009 2009 }\end{array}$ & $\begin{array}{l}\text { Tan 2010 } \\
\text { JatAL }\end{array}$ & \\
\hline $\begin{array}{l}\text { BSL initiated for babies } \\
\text { recorded as SGA }\end{array}$ & $92.3 \%(12 / 13)$ & $100 \%(16 / 16)$ & $96.6 \%(28 / 29)$ \\
\hline
\end{tabular}

The guideline was not always followed correctly for babies identified as LGA (Table 12). The guideline states that LGA babies ( $>95^{\text {th }}$ centile) by CBWC where the "possibility of diabetes in pregnancy has not been excluded" need to have BSL monitoring. Therefore, if the mother had a negative polycose or negative glucose tolerance test (GTT) in pregnancy, then BSL were not required. This was followed correctly 50\% (7/14) of the time. Seven babies had BSL done unnecessarily, as the mother had a negative diabetes screen in pregnancy. 
Table 12. Babies recorded as LGA and whether BSL were initiated according to the guideline

\begin{tabular}{cccc}
\hline & $\begin{array}{c}\text { Negative } \\
\text { diabetes screen }\end{array}$ & $\begin{array}{c}\text { BSL } \\
\text { initiated }\end{array}$ & $\begin{array}{c}\text { Guideline } \\
\text { followed correctly }\end{array}$ \\
\hline $\mathbf{1}$ & $\mathrm{Y}$ & $\mathrm{N}$ & $\mathrm{Y}$ \\
\hline $\mathbf{2}$ & Unknown & $\mathrm{Y}$ & $\mathrm{N}$ \\
\hline $\mathbf{3}$ & $\mathrm{Y}$ & $\mathrm{Y}$ & $\mathrm{N}$ \\
\hline $\mathbf{4}$ & Unknown & $\mathrm{Y}$ & $\mathrm{Y}$ \\
\hline $\mathbf{5}$ & $\mathrm{Y}$ & $\mathrm{N}$ & $\mathrm{Y}$ \\
\hline $\mathbf{6}$ & $\mathrm{Y}$ & $\mathrm{N}$ & $\mathrm{N}$ \\
\hline $\mathbf{7}$ & $\mathrm{Y}$ & $\mathrm{Y}$ & $\mathrm{N}$ \\
\hline $\mathbf{8}$ & $\mathrm{Y}$ & $\mathrm{Y}$ & $\mathrm{Y}$ \\
\hline $\mathbf{9}$ & Unknown & $\mathrm{Y}$ & $\mathrm{N}$ \\
\hline $\mathbf{1 0}$ & $\mathrm{Y}$ & $\mathrm{Y}$ & $\mathrm{Y}$ \\
\hline $\mathbf{1 1}$ & Unknown & $\mathrm{Y}$ & $\mathrm{N}$ \\
\hline $\mathbf{1 2}$ & $\mathrm{Y}$ & $\mathrm{Y}$ & $\mathrm{N}$ \\
\hline $\mathbf{1 3}$ & $\mathrm{Y}$ & $\mathrm{Y}$ & $\mathrm{N}$ \\
\hline $\mathbf{1 4}$ & $\mathrm{Y}$ & $\mathrm{Y}$ & $50 \%(7 / 14)$ \\
\hline
\end{tabular}

This finding was not surprising as most staff and LMCs were unaware they were not required to perform BSL on LGA babies if the mother had a negative diabetes screen in pregnancy.

\section{d) Ethnicity and birthweight}

The ethnicities of the babies who were identified as SGA and LGA by CBWC are shown in Table 13. It shows $75.8 \%$ of SGA babies were Maori and $57.1 \%$ of LGA babies were Maori. The upper range for a SGA baby was 3500g. The lower range for a LGA baby was $3800 \mathrm{~g}$.

Table 13. Ethnicity and birthweight of SGA and LGA babies identified by CBWC

\begin{tabular}{|c|c|c|c|}
\hline $\begin{array}{c}\text { SGA babies by } \\
\text { CBWC }\end{array}$ & Total \% & $\begin{array}{c}\text { LGA babies by } \\
\text { CBWC }\end{array}$ & Total \% \\
\hline Maori & $75.8 \%(25 / 33)$ & Maori & $57.1 \%(8 / 14)$ \\
\hline European & $24.2 \%(8 / 33)$ & $\begin{array}{l}\text { European } \\
\text { Indian }\end{array}$ & $\begin{array}{c}35.7 \%(5 / 14) \\
7.1 \%(1 / 14)\end{array}$ \\
\hline Birthweight range & $1685 \mathrm{~g}-3500 \mathrm{~g}$ & Birthweight range & $3800 \mathrm{~g}-4860 \mathrm{~g}$ \\
\hline Birthweight mean & $2680 \mathrm{~g}$ & Birthweight mean & $4333 \mathrm{~g}$ \\
\hline
\end{tabular}

Although there are other ethnic groups which birthed at the maternity unit, the only ethnicities identified in the audit as SGA or LGA by CBWC were Maori, European and Indian. Average birthing statistics for ethnic groups at the DHB 
could not be listed as this would make the DHB identifiable, other than to say there was a high Maori birthing population.

\section{Measuring whether using CBWC improved outcomes for babies}

To measure whether outcomes had improved for babies, you would need to know the number of babies admitted to the NNU for hypoglycaemia. You would need to know which of these babies had been identified using CBWC and compare this with the previous method of $<2500 \mathrm{~g}$ and $>4500 \mathrm{~g}$. There was insufficient data available to do this. The 2009 NNU statistics indicated that 23 babies received treatment for hypoglycaemia. Ten were admitted for hypoglycaemia, six were admitted with hypoglycaemia and hypothermia and seven received transitional care for hypoglycaemia. But these numbers included the babies of diabetic mothers and babies who wouldn't have met the study inclusion criteria. So there is no way to measure the effect on NNU admission rates unless the reasons for admission were specified. The 2008 NNU statistics were also insufficient to be compared to the 2009 statistics.

Babies identified as 'at risk' using CBWC received early intervention in the form of staff facilitating early, frequent and effective breastfeeds. It would be difficult to know if these babies would have had normal BSL without this type of breastfeeding support. The only evidence therefore that outcomes improved for babies since using CBWC is anecdotal.

Those involved believed that using CBWC was making a difference because it appeared there weren't any babies being admitted to the NNU with unexpected low BSL. Practitioners felt that low BSL levels were now being corrected before a baby became compromised, through implementing a proactive management approach. Over time using CBWC was reinforced as people experienced looking after 'normal' birthweight babies who weren't able to maintain BSL despite receiving extra breastfeeding support and/or breast milk and formula top-ups. Some examples of babies who required extra intervention to maintain normal BSL, who previously would not have been identified were shown in Table 9 (p. 85). There is no way to determine if these differences in outcomes are 
statistically significant, as the audit size would have been too small and the correct data wasn't available. But clinical significance could be argued if just one event such as the HDC case was prevented, as having a child with severe brain damage resulting from hypoglycaemia would be clinically significant for that family.

\section{7) Sustain knowledge use}

Nothing had been done by the DHB to sustain the use of the knowledge associated with CBWC. But there was a general feeling that attitudes had changed and people were now happy to calculate CBWC.

\section{a) Common practice}

Stakeholders believed that calculating a CBWC for every birth had become routine practice on the maternity unit.

How do you keep it going? Well we haven't really because everyone's ok about it. (Midwifery Educator)

\section{b) New staff}

As part of their orientation, new staff are informed of the policies around calculating CBWC.

As part of the orientation we give them the index of all the guidelines so that they know ... On our checklist would be setting them up on the computer IT system and making sure that they've got CBWC as well. (MM)

The CBWC is often an unfamiliar concept for new and locum staff, but it isn't as difficult to convince one person to change their practice.

Initially they were trying to convince a whole staff that this is new practice and we should do it. Whereas now that all the staff are on board it's such a culture of the ward, so it's less of a big deal when one person comes in and it's easier to say yes we do this and it's in the guideline. (Midwifery Educator) 
But when new staff questioned the use of $\mathrm{CBWC}$, it required people to revisit the evidence.

It's great now that people just do it and it is just in practice and that's why it's just a bit tedious when a new person comes in. But it keeps you on your toes if they question it you know. You can't just become complacent can you, you've got to revisit it. (Midwifery Educator)

When a new Paediatrician started who was unfamiliar with CBWC, many felt his questions were undermining the previous work that had been done.

He's just come from somewhere that doesn't do it, so he's right at the very beginning of complete disbelief about the whole thing. (Midwifery Educator)

I have since been informed that he is now convinced of the value of CBWC after experiencing their worth in clinical practice.

\section{c) Guideline reviews}

All guidelines are reviewed every two years or earlier if new evidence becomes available. The new Paediatrician was invited to help review the guideline in an attempt to make him aware of the evidence.

He has not bought into $C B W C \ldots$ and he's just not familiar with it. I've given him the readings and what I've said to him is I think he needs to help me review the guideline in March [2010]. Because it's really important that the paediatricians are fully on board with it because it is very undermining if they are not. But the other paediatricians are fully. (NNU)

\section{Summary}

The story of how CBWC were implemented at this DHB was more complex than anticipated. So a knowledge translation framework was used to describe how this evidence was put into action. The headings of the action cycle of the KTA framework were used to provide the outline for the story. The knowledge translation story began with the Midwifery Educator enquiring about CBWC. 
She then decided it was a valid tool to implement at the DHB, who provided services for a high risk birthing population. She was instrumental in facilitating the various interventions required to implement CBWC. She initially faced many challenges with implementing the knowledge, but with the release of the HDC findings and the UNICEF guidance document she was able to continue the momentum required to get CBWC fully implemented. The opposition to CBWC reduced as people began to experience looking after babies with 'normal' birthweights who were unable to maintain their BSL, despite early intervention. Consequently, calculating a CBWC for every birth became common practice and people began to accept the CBWC result regardless of birthweight. Although no formal evaluation of outcomes had occurred people felt using CBWC was making a difference by identifying 'at risk' babies who would have previously been missed.

An audit was conducted to evaluate if processes were being followed correctly and if outcomes had improved. The audit showed that although it was easy to make a mistake when calculating a CBWC, in most cases it did not make a clinical difference. But this also meant it was possible to purposely change a result so a baby was not longer identified as SGA or LGA. It showed that the CBWC was not always being recorded in the right places. It showed that the guideline was not always followed correctly, especially in regards to the management of LGA babies. But it was unknown whether using CBWC had actually reduced neonatal morbidity or mortality relating to hypoglycaemia at the DHB. The correct data wasn't available to measure this, which was the outcome people were most interested in. It showed that using CBWC identifies more babies as SGA and LGA, with the majority of these being Maori, who are a high risk birthing group. The following chapter discusses some of these key findings in further detail and comments on the research as a whole. 


\section{Chapter Six: Discussion}

\section{Introduction}

While dissecting the complexities of the knowledge translation story a number of interesting results were discovered. This chapter is divided into three sections and first discusses the more important findings relating to implementing and applying CBWC into practice. Some of the practical issues of using the CBWC calculator are highlighted, as well as the barriers which stakeholders found most challenging to overcome. Next, the knowledge translation process itself is discussed and the usefulness of applying the KTA framework to a clinical practice issue is explored. Finally, the chapter will reflect on the research as a whole and the limitations and significance of the study and suggest recommendations and ideas for future research.

\section{Section A: The process of implementing CBWC}

\section{1) Accuracy of the CBWC result: It's easy to use, however it's easy to make a mistake}

Everyone commented that the CBWC calculator was user-friendly but that it was prone to human error. Data was entered manually by selecting values from a series of drop down boxes, so accidental or intentional mistakes could be made. People considered the calculator itself to be valid but were concerned with the accuracy of the data entered, due to the variation in how people collected, recorded and interpreted the data used.

The audit showed that $62.7 \%$ of the time, I calculated the same CBWC result as what had been recorded. Rather than implying that $37.3 \%$ of the time the recorded result was incorrect, what this revealed was that two people looking at the same data could calculate a different result more than one third of the time. There were a number of possible explanations for this. Sometimes it appeared mistakes were made such as; selecting the wrong sex, incorrectly rounding 
birthweight, entering weeks of gestation instead of weeks and days and incorrectly recording the CBWC. In one instance, I recognised my own handwriting where I had recorded a CBWC of 32 instead of 23. Tiredness, distractions and a busy ward can contribute to making these types of mistakes. If the CBWC appears to be normal, it is unlikely the result would be re-checked. In the majority of cases the CBWC would have remained within the normal range, so the clinical care provided would have remained the same. While these types of mistakes were accidental, it was also possible to intentionally select incorrect data values so that a baby was no longer identified as 'at risk'. This in turn would have changed the clinical care provided.

A number of people talked about seeing others 'fix' a CBWC result by manipulating a data value to make a point of difference, which would then change a CBWC to a normal result. There were three instances where it appeared a CBWC $\geq 10$ was recorded, so a baby was not identified as SGA, when the result should have been $<10$. In these cases, the birthweights of the babies were $3180 \mathrm{~g}, 3300 \mathrm{~g}$, and $3100 \mathrm{~g}$. This showed that people were more reluctant to accept a CBWC result when the birthweight under the old definition would have been considered well within the normal range. People also had reservations about the accuracy of the data itself, especially the pre-pregnancy weight, height, gestation and ethnicity.

There was variation in the way that LMCs collected the pre-pregnancy weight and height. Height was either self-reported or measured by the LMC. Self reported height is usually always overestimated but within $3.5 \mathrm{~cm}$ of the measured height $75 \%$ of the time (Stewart, Jackson, Ford, \& Beaglehole, 1987). Overestimating height decreases the CBWC value. Many women did not know their pre-pregnancy weight. Sometimes the LMC would weigh the woman at booking and estimate their pre-pregnancy weight, or record the gestation and weight at booking instead. Commonly the booking weight was used in studies relating to CBWC (Gardosi, et al., 1992; McCowan, et al., 2005; McCowan, et al., 2004), but often this was not specified. Booking weight is listed as the variable to be entered on the calculator, rather than pre-pregnancy weight, which is what the DHB used. The guideline recommended using population centile 
charts if maternal details were missing. McCowan et al., (2004) suggested using average height and weight values for each ethnic group calculated from their study if these details were missing. Even though some error would be introduced, it is more accurate that using unadjusted population standards. When a data value is missing it is recommended that it should be estimated or population averages used, which is known as partial customisation (Gardosi, 2009b). In many of the studies examined, a large portion of the data could not be included as the maternal height and weight were not routinely recorded (Clausson, et al., 2001; Ego, et al., 2006; McCowan, et al., 2004).

The accuracy of gestation was also questioned and whether this should be calculated using the woman's last menstrual period (LMP) or by scan. Gardosi and Mongelli (1993) said that using scan dates was preferable to menstrual dates as there was a discrepancy of seven days or more in $20 \%$ of cases. Goldenberg and Cliver (1997) believed gestational age based on LMP alone is often not reliable as women may not always ovulate two weeks after the first day of their LMP. Also some women may not pay attention to the exact date of their LMP and some women may have period like bleeding early in pregnancy. One study showed that a dating ultrasound taken in the first half of pregnancy is generally a more accurate method of predicting the due date, as using 'certain' LMP dates had resulted in more women being induced for 'postdates' pregnancies unnecessarily (Mongelli, Wilcox, \& Gardosi, 1996). Gardosi (2009) recommends using ultrasound dates where possible even when menstrual dates are certain. The majority of studies on SGA babies relied on ultrasound to define gestational age (Goldenberg \& Cliver, 1997).

Questions were also raised regarding which ethnicity to use. Ethnicity is selfreported and the NZ calculator has the options of choosing Chinese, European, Indian, Maori, Samoan, Tongan and Other. People questioned which ethnicity to select if it was not included in this list. Tips given by Professor Lesley McCowan for selecting ethnicity for Polynesian women are to select Maori for Cook Island Maori and for non-Tongan and non-Samoan women to choose "other". And where possible you can also use other calculator versions for different countries, to see if there is an ethnicity that is more appropriate (Cowan, 
2009). If multiple ethnicities are given, then ethnicity is to be prioritised in the order of Maori, Pacific Peoples, South East Asian, Indian, Chinese, Other Asian, Other, Other European and European/Pakeha as outlined by the NZ Health Information Service (New Zealand Health Information Service, 2007).

Although the GROW software which developed the CBWC calculator is accurate and valid (Gardosi, 2009) the calculator itself is only as accurate as the data being used and the individual entering the data. The use of the calculator in practice highlighted the importance of accurate record keeping, accurate data collection, accurate data entry and writing legibly.

\section{2) Practice improved over time: You have to experience the benefit, before you believe the evidence}

The trend of the audit was that compliance with the guideline improved over the audit period. The CBWC was recorded and maternal details were collected more frequently. The CBWC was calculated more accurately and there was less manipulation of results. BSL were initiated more appropriately and procedures for LGA babies were followed more correctly. This is in opposition to most literature about changing practice where changes are often not sustained, as in the literature around handwashing practices (Larson, Bryan, Adler, \& Blane, 1997). But while initially challenged by having to use CBWC, compliance with calculating them improved over time as people began to believe the evidence. The results of the audit mirrored the attitudes and beliefs expressed by participants in the interviews and focus groups.

Some of the initial resistance was partly because people felt like they were just told to comply and they couldn't remember the education that had occurred previously. Some people claimed that CBWC weren't evidenced based. This is a common reaction when resisting change where clinicians are quick to critically scrutinise the evidence so they can dismiss it, when they feel the evidence is being forced on to them (Fitzgerald \& Dopson, 2005). However, stakeholders became more comfortable with incorporating $\mathrm{CBWC}$ into their practice as they 
were able to raise concerns and debate the issues. Successful adoption of evidence based practice depends on local stakeholders discussing and debating the evidence and making joint decisions about how it is applied (Ferlie, 2005). This improves knowledge uptake as end users then feel a sense of ownership over the guideline (Harrison, Graham, \& Fervers, 2009). Decreasing the number of BSL was an example of a negotiated change to the guideline which helped to appease stakeholders.

Accepting CBWC required stakeholders to redefine how SGA and LGA babies were identified and to move away from the cut-offs of $<2500 \mathrm{~g}$ and $>4500 \mathrm{~g}$. The term 'knowledge-destruction' is used to describe how we eliminate knowledge. The literature shows how it is difficult for people to destroy old knowledge and replace it with new knowledge (Landry, et al., 2006). Many had already rejected the $<2500 \mathrm{~g}$ and $>4500 \mathrm{~g}$ cut-offs as arbitrary and irrelevant. Before knowledge leads to behavioural change, "the newer knowledge has to actively relate to what individuals already know, including what they know through their experience" (Fitzgerald, Dopson, Ferlie, \& Locock, 2005, p. 156). Clinical experience meant practitioners had already noticed trends such as mothers from different ethnic groups having different sized babies and smaller women being predisposed to having smaller babies. So the scene had been set for the knowledge around CBWC to be assimilated into practice.

Identifying babies as SGA and LGA and identifying risk of hypoglycaemia were not new ideas. But using CBWC to identify this risk was a novel concept. The subject of hypoglycaemia was itself a controversial and contentious issue. Inevitably introducing CBWC as a tool to identifying risk of hypoglycaemia would be difficult. When the tool identified higher birthweight babies as SGA, beliefs were challenged and some regressed to the old definitions of 'normal' birthweight. But studies have shown that babies with the highest rates of perinatal morbidity and mortality are those identified as SGA by CBWC only (Clausson, et al., 2001). The higher birthweight SGA babies that practitioners were challenged by were the babies that should have been monitored more vigilantly. Rather than dismissing their birthweight as 'normal', in reality these were the babies at highest risk. 
Those implementing CBWC recall the process being incredibly challenging at times and taking longer than expected for all stakeholders to accept the changes. But over time compliance improved and people readily accepted the CBWC result regardless of birthweight. The resistance diminished as people experienced looking after babies with a 'normal' birthweight who were not able to maintain BSLs despite intervention. Ferlie and Dopson (2005) have said that the visibility of outcomes influences the success rate of adoption. The process of change before adopting a new practice was described by an awarenessagreement-adoption-adherence model (Davis \& Davis, 2010). Relating this to CBWC, the LMC or core staff became aware of the new practice, moved through a process of agreement and then an adoption of CBWC to finally adhering and conforming to the practice (Davis \& Davis, 2010). Those who had not experienced this phenomenon admitted they were less convinced about the value of CBWC.

\section{3) Did it make a difference?: Was a case similar to the HDC scenario prevented?}

Using CBWC there were 22 babies identified as SGA and eight babies identified as LGA who previously would have been missed. Details for these babies were outlined in Table 9 (p. 85) and Table 10 (p. 88). It is difficult to know what difference was made by identifying and monitoring these additional babies. When evaluating any knowledge translation process, it is often difficult to find strong evidence to support specific changes in health care delivery (Wensing, et al., 2010). It is challenging to measure how a change in practice translates into a change in patient outcomes (Rycroft-Malone \& Bucknall, 2010a). It is impossible to know whether those babies identified by CBWC only would have gone on to develop hypoglycaemia and/or brain damage if their care wasn't proactively managed from the beginning.

The NNU statistics were insufficient to measure how admissions had changed as a result of CBWC being implemented. There was anecdotal evidence that there were no longer admissions to the NNU of babies with unexpected very low BSL. 
There were a few examples of babies who were not able to maintain normal BSL despite early recognition and early intervention, who required further intervention in the NNU. Perhaps in these cases we can say it did make a difference and possibly prevented a baby from becoming very unwell. But in reality the data for these outcomes weren't available. To measure if implementing CBWC really did make a difference, it may be more appropriate to measure knowledge use versus the impact of knowledge use. There are three ways that knowledge can be used which are: instrumental, conceptual and symbolic (Straus, Tetroe, Graham, Zwarenstein, \& Bhattacharyya, 2009). These can be measured to see whether the knowledge is making a difference, rather than measuring the impact of the knowledge use (Straus, Tetroe, Graham, et al., 2009).

Instrumental knowledge is about measuring the change in practitioner behaviour, rather than measuring a change in health status (Graham, Bick, Tetroe, Straus, \& Harrison, 2010). "When the implementation intervention targets a clinical behaviour for which there is strong evidence of benefit, it may be appropriate to measure outcome only in terms of whether the behaviour occurred, making practitioner behaviour an indicator of the outcome measure or endpoint" (Hakkennes \& Green, 2006, p. 8). Although clinical outcomes could not be measured at the patient (baby) level, the change in behaviour of stakeholders could be measured. Maybe in this case, the primary outcome was compliance with the guideline rather than the rates of neonatal morbidity and mortality. The audit demonstrated all practices relating to compliance with the guideline improved over the audit period.

Conceptual knowledge is about using knowledge to get people to change the way they think about issues (Straus, Tetroe, Graham, et al., 2009). Over time people's attitudes changed and people believed calculating a CBWC had become routine practice. But self efficacy was higher than what was in reality happening, as the audit showed there was room for improvement. Symbolic use of knowledge, also known as persuasive knowledge use, has to do with using knowledge to influence policy (Straus, et al., 2010). The knowledge of the adverse HDC event was used to persuade stakeholders that implementing CBWC 
was urgently needed. Although it is unknown whether calculating a CBWC would have actually made a difference in the outcome for the baby in the HDC case.

One difficulty with evaluating knowledge translation processes is that often a clear definition isn't given for what a clinically worthwhile improvement is (Majumdar, 2009). When the DHB implemented CBWC, they hoped that it would prevent a scenario such as the HDC case from occurring at their hospital. But at the beginning of the implementation process they had not defined how this would be measured. It would have been helpful to decide what the worthwhile clinical difference would have needed to be to consider the intervention was working (Majumdar, 2009). Although specific numbers couldn't be evaluated and there were no relevant statistics to compare, stakeholders believed the use of CBWC had made a difference. One could easily argue that preventing a single baby from developing brain damage was clinically significant versus statistically significant. Although this would also need to be balanced with the increased intervention for babies required.

CBWC made a difference by introducing a more accurate method of identifying SGA and LGA babies. It made a difference by changing people's attitudes towards managing hypoglycaemia and the care stakeholders provided was more proactive. It made a difference for those babies who required admission to the NNU when they weren't able to maintain normal BSL on the postnatal ward. These babies were able to receive early treatment and intervention before becoming symptomatic. According to the studies on customisation, these were the babies at highest risk of perinatal morbidity and mortality, who previously would have been missed.

\section{Summary}

Implementing CBWC at any maternity unit will be a challenging endeavour because most practitioners are unfamiliar with CBWC and hypoglycaemia is a controversial topic in of itself. But the concepts behind the development of 
CBWC, such as women of different ethnicities having different sized babies, supports the knowledge that practitioners have experienced in practice. Over time attitudes can change which can change the way one chooses to practice. This can happen as practitioners recognise the value of CBWC in identifying babies requiring intervention that would have previously been missed.

\section{Section B: The knowledge translation framework}

\section{1) The KTA framework is unnecessarily complex; however, knowledge translation is a complex process}

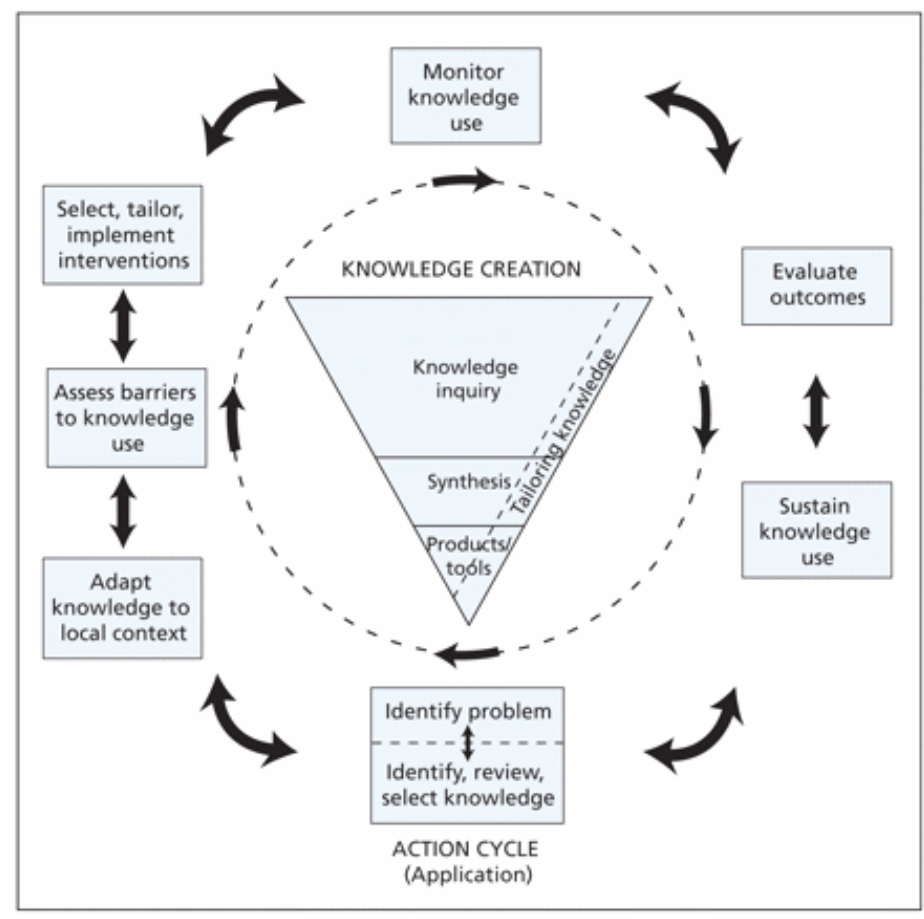

Figure 4. The knowledge-to-action framework (Straus, Tetroe \& Graham, 2009, p. 167)

The KTA framework itself was readable and seemed to be set out in a logical, step-by-step manner. It appeared user-friendly and at face value seemed to represent knowledge translation as a straightforward, linear process. But the developers of the framework explained that "in reality, the process is complex and dynamic, and the boundaries between these two concepts and their ideal phases are fluid and permeable" (Graham, et al., 2006, p. 18). Complexity was 
demonstrated in the diagram by the use of double sided arrows. But this was only truly understood when the framework was applied to a 'real world' clinical practice issue. The timing of events and a number of people and processes facilitated knowledge translation occurring. A closer examination of the literature found the authors acknowledged how potentially inadequate the framework could be.

"While this representation suggests circularity or a sequence of phases that need to be taken in order, this is not how implementation projects unfold in "real life". They are often chaotic, and move forward in an erratic manner with continuous course corrections as the action phases accommodate to contextual factors. Perhaps a better representation of our framework would be the probabilistic atomic model, where the action phases are like electrons around the nucleus of knowledge generation - and the contextual factors influence where a given phase might be at a specific time" (Graham \& Tetroe, 2010, p. 214)

The KTA framework lacked the ability to visually represent how complex the process of knowledge translation truly was.

The implementation of CBWC at a NZ DHB was evaluated for this research. In wanting to interpret what happened in a meaningful way, the KTA framework was used as a research tool to help describe the knowledge translation process that had occurred. Applying the KTA framework in this way was not as straightforward as had been presumed. It was not as simple as merely placing events as they happened within the framework headings. The reality was that the action phases may occur sequentially or simultaneously (Graham, et al., 2006) and a degree of flexibility was required when using the framework. During the data analysis it was sometimes hard to separate the individual parts of the framework. It was often difficult to decide which heading a particular finding should be placed under. Findings often overlapped and the way each framework heading was defined was also open to interpretation. In using the framework an obligation was felt to put data under each individual heading. Interpreting and describing the implementation process using the framework became a confusing and frustrating exercise. While the framework wasn't able to visually 
demonstrate the complexity of knowledge translation, on the other hand there were too many headings.

By reworking the action cycle and combining headings, the KTA framework could have been simplified further. In the literature, when phases in the action cycle were discussed, they were often combined as if they were one topic. For example, 'Adapting knowledge' and 'Identifying barriers' were often discussed together (Straus, et al., 2008; Straus \& Holroyd-Leduc, 2008). Only subtle differences were found between 'Monitor knowledge use' and 'Evaluate outcomes' and the concept of 'tailoring interventions' could be likened to 'Adapting knowledge to local context'. Headings and concepts could be combined to make a simplified version of the action cycle in the KTA framework (Figure 8). This version condenses the number of headings from seven to five. The arrows in the middle of the figure indicate the processes can also happen non-sequentially.

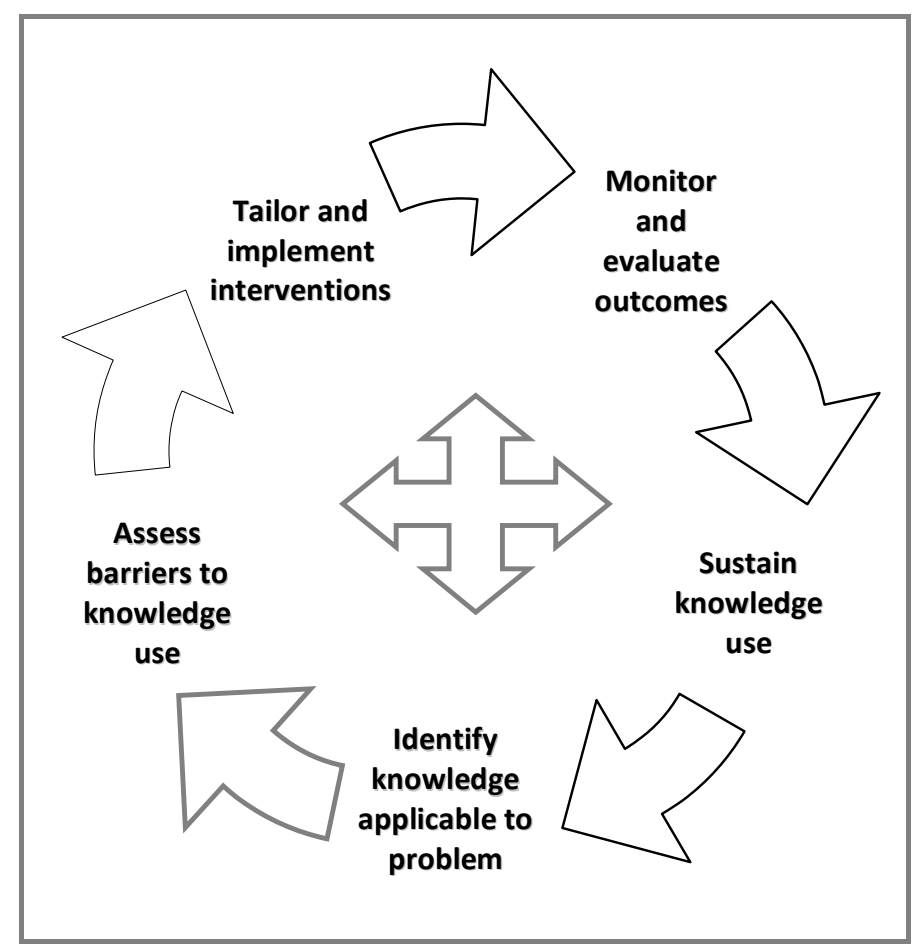

Figure 8. Simplified action cycle adapted from the KTA framework

The nature of conceptual frameworks are that they "are in a continual state of development as new evidence emerges which may challenge and augment their 
contents" (Rycroft-Malone \& Bucknall, 2010b, p. 28). Therefore, it is acceptable to adapt the framework as a result of this research experience. Simplifying the action cycle of the framework makes it less detailed and perhaps even more userfriendly. But this simplified version is also unable to reflect the complexity of the knowledge translation process. Although it is unable to demonstrate the complexity experienced within a one-dimensional diagram, the KTA framework is still a valuable tool.

\section{2) Knowledge translation can happen without a framework, but using the KTA framework can help to complete the process}

The DHB did not use the KTA framework when implementing CBWC at their maternity unit. It was applied retrospectively as a research tool to aid with the data analysis. Instead, the DHB followed their normal processes when applying any new evidence to a guideline eg emailing guideline changes, perinatal meeting presentations etc. This demonstrated that knowledge translation can happen without the use of a framework. But what a framework can do is highlight the ideal phases needed which can assist stakeholders to complete the knowledge translation process.

Without using a framework the DHB were able to complete a number of the ideal phases proposed by the KTA framework. But there were also a number of steps which weren't done which means their knowledge translation process was incomplete. Barriers to using the knowledge of CBWC were not initially assessed. So the interventions employed were not specifically designed to address any potential barriers stakeholders might have to implementing CBWC into their practice. Routine processes were followed for updating any guideline or policy at the DHB. But Wensing et al., (2010) explained that it is important that knowledge translation interventions are specifically tailored to barriers, in the same way that a clinical treatment is tailored to a specific health problem. The level of opposition to CBWC was unexpected. By tailoring the interventions perhaps it wouldn't have taken as long for stakeholders to accept the evidence. After implementing CBWC nothing had been done to formally monitor knowledge use, evaluate outcomes and sustain knowledge use. The Midwifery 
Educator had wanted to evaluate outcomes but was unable to. Often the predicament is having the time and funding to properly assess change processes (Davies \& Edwards, 2009). It is also acknowledged that sustainability is rarely addressed even though it is recommended in the early stages when interventions are being designed (Davies \& Edwards, 2009).

This research attempted to complete the knowledge translation process by undertaking the phases of the action cycle that had not been done. But this wasn't entirely possible as the required data wasn't available. It was not possible to truly evaluate what difference, if any, implementing CBWC had made on the numbers of babies experiencing morbidity and mortality relating to hypoglycaemia. Ideally applying a framework early in an implementation process can help to guide the process and focus attention on what is important (Rycroft-Malone \& Bucknall, 2010a). In this way decisions can be made from the beginning about what outcomes are most important and what data needs to be collected to be able to measure this.

The DHB did not need to use a framework to facilitate implementing CBWC. But the knowledge translation process was incomplete because the DHB did not evaluate whether CBWC were being used as it was intended and did not evaluate the outcomes resulting from the change in practice. This is where a framework may have been helpful. Frameworks should facilitate, not constrain the implementation process (Rycroft-Malone \& Bucknall, 2010a). It can help you know what is required to make the process complete. When used at the planning stages of implementation it can illustrate what is needed to complete the process. But one thing that did become evident from the implementation process at the DHB was the need for a champion.

\section{3) A champion facilitates the knowledge translation process}

A champion facilitates knowledge translation by maintaining the momentum needed to advance change. It is important that organisations invest in someone who is responsible for ensuring that midwifery practice is evidence-based, as change cannot occur without a champion (Melnyk, Fineout-Overholt, Stone, \& 
Ackerman, 2000). Identifying people who are enthusiastic about the issue, can help sell new ideas (Rycroft-Malone \& Bucknall, 2010a). In this knowledge translation process the champion was the Midwifery Educator.

The Midwifery Educator was the person mainly responsible for updating clinical guidelines at the maternity unit. In addition to facilitating all Quality related events, she worked clinical shifts. Her workload would have been covered by a number of employees at a larger DHB. When she first heard about CBWC through her role as the local PMMRC Coordinator, she investigated how it might be used at the maternity facility to help better identify SGA and LGA babies. After consulting with stakeholders she included CBWC in the relevant guidelines. But it wasn't until staff and LMCs were expected to start calculating a CBWC for every birth that people starting opposing the change. With the timely release of the HDC findings and the UNICEF guidance document, the opportunity presented itself to fully implement CBWC through further facilitating a variety of interventions. The Midwifery Educator was identified by all stakeholders as being instrumental in the whole process. Undoubtedly without her hard work CBWC wouldn't have been put into action at the DHB.

\section{Summary}

Knowledge translation is a complex process that is facilitated by a number of phenomena, including a champion. It can happen without the use of a framework. But what using a simple framework can do is help to identify the phases required to complete the process.

\section{Section C: Reflections on the research as a whole}

\section{1) It was not possible to do a process-outcome evaluation}

Chapter Four (p. 36) outlined the research approach, design and methods used. The research was described as a process-outcome evaluation. The aim was to evaluate peoples experiences of the events that occurred and the extent to which processes were being followed correctly, as well as evaluating outcomes 
resulting from introducing CBWC. Although the intention was to perform a process-outcome evaluation, in reality, the study was a process evaluation. The data wasn't there to evaluate if CBWC actually did improve clinical outcomes and make a difference for babies. It was unknown whether any scenarios, similar to the HDC case, had been prevented since CBWC were implemented. Evaluating the extent to which the program was implemented was able to explain some of the outcomes of the audit (Owen, 2006). But it was not possible to evaluate the real difference it made in influencing the rates of perinatal morbidity and mortality relating to neonatal hypoglycaemia, which was disappointing.

\section{2) Using CBWC facilitated a proactive approach to managing hypoglycaemia and made practitioners more aware of the bigger picture of risk assessment}

Using CBWC to identify SGA and LGA babies was just one factor to consider in identifying babies at risk of hypoglycaemia. In the beginning, CBWC was the only issue people focused on during some heated discussions at perinatal meetings. Those implementing CBWC explained that the changes in the guidelines weren't just about CBWC. The changes were also about the bigger picture of risk assessment and the DHB being more proactive in their approach to managing neonatal hypoglycaemia.

The CBWC result was included in a flowchart used for the ongoing assessment of babies at risk of hypoglycaemia (Appendix L). The flowchart stressed that babies should be reviewed prior to each feed for 24 - 48 hours, even after two normal BSLs were recorded. Feeding charts (Appendix M) were also used which required staff to assess the quality of each breastfeed. Both of these tools emphasised a proactive management approach towards babies at risk of hypoglycaemia. There was a recognition that since the introduction of CBWC, stakeholders were more aware of hypoglycaemia and were intervening before a baby become symptomatic. Eventually, people viewed the CBWC as more than just a number and saw how it related to the environment in which the baby was expected to thrive. People began to see how a baby's CBWC related to the 
bigger picture of risk assessment and the care and education provided in the antenatal, intrapartum and postnatal periods.

Stakeholders began to understand that CBWC wasn't just about hypoglycaemia. They noticed relationships between CBWC and smoking, obesity, diet, diabetes screening, drugs and alcohol in pregnancy and safe sleeping messages. The CBWC seemed to correlate with high risk birthing groups as $75.8 \%$ of SGA babies were born to Maori mothers. Maori pregnant women are over-represented in socioeconomic deprived areas (New Zealand Health Information Service, 2007). Smoking rates for Maori women of childbearing age are more than double that of non-Maori, peaking at $61 \%$ for women between the ages of $20-24$ years (The Quit Group and the Ministry of Health, 2009). The rates of Maori babies dying of SUDI (Sudden Unexplained Death in Infancy) are significantly higher than other ethnic groups (Child and Youth Mortality Review Committee, 2009). Gestational diabetes is diagnosed in $6 \%$ of pregnant Maori women, which is double that of NZ European women (Health Research Council of New Zealand, 2010). Maori women are "1.7 times more likely to be obese than ... women in the total population" (Ministry of Health, 2008, p. 111). Although not audited, it would have been interesting to have investigated the smoking status, socioeconomic status and other health risk behaviours of the women having a SGA baby. Obesity is defined as a BMI $>30 \mathrm{~kg} / \mathrm{m}^{2}$ (C. T. R. U. Ministry of Health, 2009). In the study $31.8 \%$ (7/22) of mothers with a SGA baby identified by CBWC only, were obese and $62.5 \%$ (5/8) of the mothers having a LGA baby identified by CBWC only, were obese. Maternal obesity in pregnancy is associated with many risks (Yu, Teoh, \& Robinson, 2006). People recognised these types of risk factors were often associated with the mothers of babies identified by CBWC as SGA or LGA.

When a baby was born with a CBWC of 0 , people would ask whether a CGC chart was completed antenatally. People questioned why the baby failed to grow to its optimum size and what factors were involved. These cases were often discussed at perinatal meetings, especially when there was a poor outcome for the baby. The discussions were around whether a low CBWC was expected and what could have been done differently. Babies born with a low CBWC 
reinforced the importance of using CGC charts in pregnancy for those LMCs who weren't routinely using them as had been recommended.

\section{3) Reflection on the HDC case}

The implementation of CBWC was described by an LMC as a "knee jerk reaction" to the HDC case which was based on "fear". Although CBWC had already been included in the relevant guidelines, the HDC report acted as the catalyst to accelerate the implementation process. Areas chosen for policy development are often in response to an adverse incident. Policy is then developed outlining how a specific practice matter should be approached which aims to prevent future incidents (Boogaerts, Grealish, \& Ranse, 2008). Some criticise that this can be limiting when the response is to a single event "where professional judgement (or lack thereof) may have been a significant factor" (Boogaerts, et al., 2008, p. 52). The HDC case was a single event which occurred at another DHB. But the Clinical Director of Child Health was charged by the clinical board to show how a case such as this would be prevented at their DHB. Using CBWC became one answer presented to the clinical board to better identify babies at risk of hypoglycaemia.

It is unclear whether calculating a CBWC would have even made a difference in the HDC case. Maternal details were not given in the report, so a CBWC could no be calculated. Sex-adjusted population based centiles used at the DHB in this study gave a BWC sitting around the $10^{\text {th }}$ centile (Appendix O). The baby was delivered by emergency caesarean section due to foetal distress in early labour. There were concerns around whether the baby had adequate reserves to tolerate a long labour. The Apgars were 7 at one minute and 10 at five minutes and the baby received facial oxygen. The mother was taking the anti-depressant Aropax, so the baby was at risk of hypoglycaemia, regardless of its birthweight. Aropax is not recommended in pregnancy unless the potential benefit for the mother outweighs the possible risk to the baby (GlaxoSmithKline NZ Ltd, 2010). It is recommended that babies of mothers on Aropax are observed closely for hypoglycaemia and that complications were most likely to occur in the first 24 hours (GlaxoSmithKline NZ Ltd, 2010). The mother was also a smoker. The baby most likely would have had normal BSLs in the first 24 hours as it was 
initially feeding well. By Day three it was symptomatic of hypoglycaemia, but it is unknown how long a baby can be hypoglycaemic before becoming symptomatic (World Health Organization, 1997). The hypoglycaemia had gone unrecognised and the baby deteriorated to the point of becoming brain injured, despite late intervention. There were several factors, other than the baby's birthweight, which may have contributed to the baby's inability to produce an appropriate metabolic and endocrine response to counterregulate the hypoglycaemia that occurred (Cornblath, et al., 2000). The baby's birthweight may not have been the greatest risk factor for hypoglycaemia. Calculating a CBWC alone may have not made a difference, as other risk factors were also not identified.

When a policy is developed in reaction to an adverse event occurring this can sometimes obscure the real issue which is often the cause of the incident (Boogaerts, et al., 2008). The HDC report played a significant role in facilitating CBWC to be fully implemented at the DHB, as a proposed way to prevent a case such as this occurring at their hospital. But it is unknown whether calculating a CBWC would have really made a difference in this scenario. The real issue might have been in the delay in recognising the baby was no longer feeding appropriately and the non-recognition of a number of factors that put the baby at risk of hypoglycaemia. The introduction of any instrument is only effective when used in conjunction with good clinical observations and judgment. The midwifery expert and Commissioner discussed the use of CGC as a better guide to identify babies at risk. But in the context of this case, what they actually should have been recommending was the calculating of a CBWC at birth, as they were discussing identifying birthweight as a risk factor for hypoglycaemia. So even the experts were unsure as to what they were referring to. This showed how unfamiliar people were with using CGC and CBWC in clinical practice.

\section{4) Reflection on conducting the research}

I wanted to do a Masters thesis so I could gain the experience of completing a piece of research. I was naively unaware of how challenging the process would be. From initially writing a research proposal and gaining ethical approval, to collecting and analysing data, to discussing findings and results, I often felt like I 
was stumbling along the way. The biggest lesson learned was that research takes much longer than expected and I was constantly having to adjust the research timeline I had set myself. I believe that this research project has given me a new set of skills which will aid me in my future educational and professional endeavours. Overall, I felt the research went well and despite the challenges I accomplished what I wanted to achieve. Completing this research ended up being more about the journey and the learning acquired along the way, as much as it was about CBWC, evaluation and knowledge translation. This thesis has shown me that I can transform data into something meaningful, which can be applied in a practical way to the clinical environment and contribute to the field of midwifery knowledge.

\section{Limitations}

The study was limited by its size and scope. This was a small scale research study completed to fulfill the requirements of a Masters Thesis. The findings of the study may not be applicable in other scenarios such as a larger DHB with differing population characteristics. If fewer methods were employed I perhaps would have been able to achieve a more in-depth analysis and discussion rather than an overview of several areas. Several methods were required to answer all of the objectives I wanted to achieve. No statistical analysis was performed as the audit sample size of 303 births was likely too small to determine statistical significance and was beyond the scope of the research project.

Stakeholders were most interested in learning whether the use of CBWC had made a difference by reducing neonatal morbidity and mortality associated with hypoglycaemia. Determining this outcome was unachievable as the data wasn't available to answer the question. It would have required the NNU recording more specific data around the reasons for admissions relating to hypoglycaemia. This was unfortunate as one of the main reasons why it was implemented and the Clinical Director of Child Health was so passionate about it, was that the DHB wanted to prevent babies developing brain damage related to hypoglycaemia. But anecdotally it appeared no babies with really low unexpected BSLs were 
being admitted to the NNU and no one remembered a baby sustaining a brain injury relating to hypoglycaemia.

One disappointment was that the Clinical Director of Child Health was not forthcoming to be interviewed. He commented that he would have nothing further to add from what could be obtained from the other interviews. He said that his role was to only support the Midwifery Educator and NNU Quality Coordinator in the writing of the relevant guidelines. It is unknown why the Clinical Director minimised his role in the process, as he was highlighted by many stakeholders as a key person to talk to. Nobody knew why this HDC case specifically was highlighted by the clinical board or whether it was just standard practice to review all HDC cases. The Clinical Director would have been able to answer this question and shed light on other aspects of the implementation process.

Although the sample size was small, the research still contributes to the knowledge around the practical use of CBWC in a clinical environment. It also makes a contribution by commenting on the usefulness of applying the KTA framework to a clinical practice issue.

\section{Section D: Significance/Recommendations/Ideas for further research}

\section{1) Significance}

It was recognised that "further studies in other populations are required to determine the generalisability of these earlier studies of customised centiles and their role in prediction of morbidity in general populations" (McCowan, et al., 2005, p. 1027). This research was a small scale study and was only able to provide anecdotal evidence that using CBWC decreased neonatal morbidity at the DHB. But there still appeared to be a number of firsts associated with the research.

This was the first piece of research to investigate the use of CBWC as an assessment tool for identifying babies at risk of developing hypoglycaemia due 
to birthweight. This research was also the first to explore issues relating to the practical application of the CBWC calculator at a maternity unit and the challenges involved with changing the way people practiced. This research was also unique in that it applied the KTA framework retrospectively to a clinical practice issue and was able to comment on the usefulness of using the KTA framework in this way.

\section{2) Recommendations}

Calculating a CBWC relies on accurate data entry. The calculator requires users to select data values from a series of drop down menus and mistakes can easily be made. Incorrect data values can also be purposefully selected to manipulate a CBWC result so a baby no longer requires BSL monitoring. To remove the temptation to change a result, it would be useful if the computer system allowed for a woman's booking visit data to be pre-entered. At the time of birth the gestation would be automatically calculated and only the birthweight and baby's sex would be entered to generate the CBWC. The hospital computer system was not designed this way. But I have worked at another DHB where the mother's booking information is pre-entered, which makes it more difficult for practitioners to go back and change the original data entered. Double checking data entry would also help to decrease the number of mistakes.

The Maternity Unit Manager had emailed other maternity managers in NZ to enquire what methods were being used to identify SGA and LGA babies. From the few responses received, she learned other DHBs were using the $<2500 \mathrm{~g}$ and $>4500 \mathrm{~g}$ cutoffs, or a chart with cut-off weights for varying gestations based on population BWC charts. No other DHBs were using CBWC. The evidence shows that using customised centiles is preferable to using population based centiles (Ego, et al., 2006). So using CBWC is more desirable than using cut-off weights based on population BWC charts. The evidence that gestation influences whether a birthweight should be considered 'normal' (World Health Organization, 1961) indicates that no DHB in NZ should be using the cut-off points of $<2500 \mathrm{~g}$ and $>4500 \mathrm{~g}$ to identify SGA or LGA babies. These values are arbitrary and out-dated and do not take into consideration a number of variables 
which are known to affect the potential optimal growth of a baby, most importantly gestation and maternal weight. Using these out-dated cut-off points will result in a number of 'at risk' babies being missed.

The LMC should consider calculating a CBWC as part of the initial newborn assessment and part of the primary care provided. The LMC should be aware of the CBWC result before handing over care of the baby to core staff. This requires the $\mathrm{LMC}$ to either calculate the $\mathrm{CBWC}$ themselves or to ask a staff member to assist them. The LMC should be the one who informs the woman and her family of a CBWC result if the baby requires BSL monitoring. The LMC should also initiate the appropriate paperwork and care plan and forward this information to core staff. Where the woman has been handed over to secondary care prior to or during labour, the core staff would take over these responsibilities. If LMCs and core staff communicate their roles clearly and work as a team, there will be less missed opportunities where the calculating of a CBWC is delayed.

If the Perinatal Maternal Mortality Review Committee recommend CGC to be completed for all pregnancies in $\mathrm{NZ}$, then it is reasonable to recommend calculating a CBWC for every birth. The evidence which lead to the development of the GROW software is valid for both CGC and CBW. A CGC can only predict birthweight and estimate growth restriction and macrosomia, whereas a CBWC is calculated using the actual birthweight. If customisation is a valid argument for the provision of antenatal care, then it should be considered just as valid in the postnatal period. If practitioners are expected to act upon the results of plotted CGC charts in pregnancy, then they should be expected to act upon the results of a CBWC calculated at birth. The two are not mutually exclusive. Completing both a CGC and calculating a CBWC will help to determine how closely correlated the two results are.

When implementing new evidence into a guideline, using a knowledge translation framework can help stakeholders to identify all the steps required to complete the process. This is particularly important when the evidence involves an unknown or novel practice. Identifying a 'champion', who is supported by 
management, is also crucial in facilitating the implementation process. Also any major change in a guideline should be evaluated within a given time period, to assess whether processes are occurring as intended and whether the outcomes are producing the desired results.

These recommendations highlight the need for further research to be conducted to identify the difference that CBWC really makes.

\section{3) Ideas for further research}

There are numerous opportunities for further research relating to the use of CBWC in clinical practice and the relatively new field of knowledge translation.

If CBWC are to be used as a method for determining the risk of SGA and LGA babies developing hypoglycaemia, then more research needs to be performed to provide the evidence for defining monitoring threshold limits. The DHB studied monitored babies with a CBWC $<10$ or $>95$ (where the mother had not had a negative diabetes screen in pregnancy). But it is possible that these thresholds could be narrowed, for example to $<5$ or $>97$. But further research is needed to determine what these cut-off points could safely be.

Research needs to be performed to determine the correlation between fundal height measurements on a CGC and the CBWC at birth. This way the predicted birthweight can be compared to the actual birthweight. This will confirm how accurate the CGC are in determining birthweight based on plotted fundal height measurements.

The KTA framework needs to be applied in more clinical practice settings which may further validate the use of the framework as a clinical practice tool. Ideally the framework would be applied in a prospective, longitudinal manner, so that the different phases of the implementation process can be informed as it happens (Spyridonidis \& Calnan, 2011). In this study the framework was not used by the DHB, but it was applied retrospectively to aid with the data analysis and presentation of the knowledge translation story. 


\section{Conclusion: Revisiting the Research Question}

The first aim of this study was to identify and critique the evidence relating to the use of CBWC. The evidence demonstrates that using customised standards versus population based standards, improves the identification of babies at greater risk of perinatal morbidity and mortality. The literature showed that the variables gestation and maternal weight have the greatest physiological effect on birthweight. Ethnicity, maternal height, parity and the baby's sex also have a significant effect on birthweight. Smoking has a significant pathological effect on optimal foetal growth and was not included in the development of the GROW software. GROW was used to create CGC and the CBWC calculator. While the evidence supporting the use of CBWC has been available for a number of years, they are not commonly used within NZ DHBs. There has been a delay in getting this knowledge translated into action.

The second aim of this study was to describe how CBWC were implemented at one NZ DHB. Data from interviews and focus groups formed the basis of the implementation story and additional documentation helped to verify specific events and dates. A knowledge translation framework was used retrospectively to provide a theoretical foundation and describe the process in a meaningful way. The Midwifery Educator was the champion who facilitated various interventions to implement CBWC. CBWC became fully implemented after the release of a HDC report and a UNICEF guidance document, which catalysed the momentum required for this to happen.

The third aim of the study was to evaluate the processes followed when utilising CBWC. Qualitative data described the attitudes and beliefs of stakeholders towards CBWC and an audit was conducted to see if the guideline was being followed correctly. Initially practitioners were challenged by the evidence for CBWC, especially when babies of 'normal' birthweight were being identified as SGA or LGA. But over time people came to accept the CBWC result regardless of birthweight, when they experienced caring for babies who were unable to 
maintain normal BSL despite early intervention. This was mirrored by the audit results where compliance to the guideline improved over time.

The fourth aim of the study was to identify outcomes relating to the babies identified as SGA or LGA using CBWC. It was unknown whether perinatal mortality and morbidity had decreased since implementing CBWC, as the appropriate data was not available. But the DHB had become more proactive in their management of hypoglycaemia and identifying risk. Practitioners were intervening earlier instead of waiting for babies to become symptomatic. There was anecdotal evidence that using $\mathrm{CBWC}$ was making a difference in identifying babies who would have previously been missed. It is unknown whether a scenario similar to the HDC case had been prevented, although there were some examples of possible near misses found in the audit.

The fifth aim of the study was to evaluate the usefulness of the knowledge-toaction Framework when applied to a clinical practice issue. Applying the framework in a retrospective manner was sometimes difficult and confusing. This highlighted the fact that knowledge translation is a complex process which is inadequately demonstrated by a one-dimensional diagram. But what using a framework can do is identify the ideal phases needed for knowledge translation to be completed. It also highlighted the importance of a champion to facilitate the implementation process.

The significance of this study relates back to the original research question which was:

How was the evidence behind the use of $\mathrm{CBWC}$ put into practice at one $\mathrm{NZ}$ $\mathrm{DHB}$ and what are the outcomes resulting from translating this knowledge into action?

Although this research is a small-scale evaluation, it contributes to knowing how the evidence for CBWC can be translated into action. This is the first study which describes the practical application of CBWC in a clinical practice setting. Consequently, this research may be of interest to other maternity units who are 
considering whether to implement CBWC. But there are still a number of issues around CBWC that need to be explored. This includes determining the appropriate cut-off points to be used to identify SGA and LGA babies by CBWC and identifying the real difference CBWC makes in reducing morbidity and mortality outcomes for babies at risk of hypoglycaemia. 


\section{Appendices}

\section{Appendix A: Ethics Approval}

Health

and

Disability

Ethics

Committees

4 November 2009

Ms Malia Lardelli

25 Griffiths Grove

Newlands

Wellington 6037
Northern Y Regional Ethics Committee

Ministry of Health

$3^{\text {rd }}$ Floor. BNZ Building

354 Victoria Street

PO Box 1031

Hamilton

Phone (07) 8587021 Fax (07) 8587070

Dear Malia

Evidence into Practice: An evaluation of the implementation of customised birthweight centiles at one New Zealand DHB.

Investigator: Malia Lardelli Supervisor: Joan Skinner

Ethics Ref: NTY/09/69/EXP

The above study has been given ethical approval by the Deputy Chairperson of the Northern Y Regional Ethics Committee.

Approved Documents

Research Proposal

Information sheet and consent form for interview version 1 dated 21/07/2009.

Information sheet and consent form for focus group version 1 dated 21/07/2009.

Interview question guide.

Focus group question guide LMCs .

Focus group question guide maternity staff midwives/nurses

Invitation poster for focus group

Audit tool.

Progress Reports

The study is approved until 30 April 2011. The Chairperson will review the approved application annually and notify the Investigator if they withdraw approval. It is the Investigator's responsibility to forward a progress report prior to ethical review of the project in 4 November 2010. The report form is available on

http://www.newhealth.govt.nz/ethicscommittees. Please note that failure to provide a progress report may result in the withdrawal of ethical approval. A final report is also required at the conclusion of the study.

Amendments

It is also a condition of approval that the Committee is advised if the study does not commence, or is altered in any way, including all documentation eg advertisements, letters to prospective participants.

Please quote the above ethics committee reference number in all correspondence.

It should be noted that Ethics Committee approval does not imply any resource commitment or administrative facilitation by any healthcare provider within whose facility the research is to be carried out. The organisation may specify their own processes regarding notification or approval.

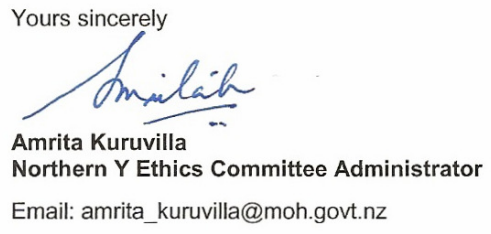




\section{Appendix B: DHB Approval}

CORPORATE SERVICES DEPARTMENT
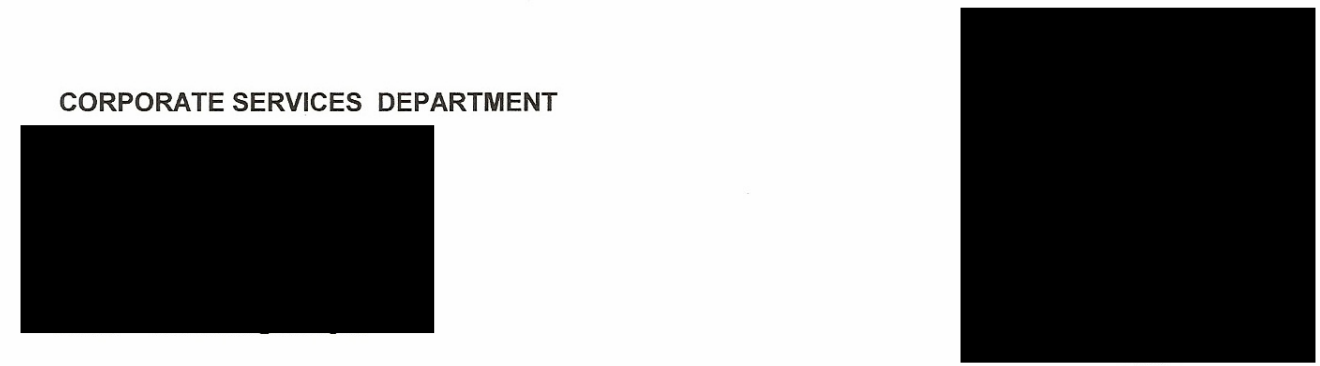

Malia Lardelli

25 Griffiths Grove

Newlands

WELLINGTON 6037

Dear Malia

RESEARCH WORK THE IMPEMENTATION OF CUSTOMISED BIRTHWEIGHT CENTILES AT ONE NZ DHB"

EVIDENCE INTO PRACTICE : AN EVALUATION OF

ETHICS REF : NTY/09/69/EXP

This letter confirms that on 09/11/09 an email fron $\quad$-Chief Medical Advisd oted th received no comments related to the above proposea course or study and that he saw no reason to delay the research.

It was noted that your study had obtained approval from the Northern Y Regional Ethics Committee Deputy Chairperson on the 04/11/09.

Congratulations on your successful bid and please note that we would like to receive a copy of your completed research report.

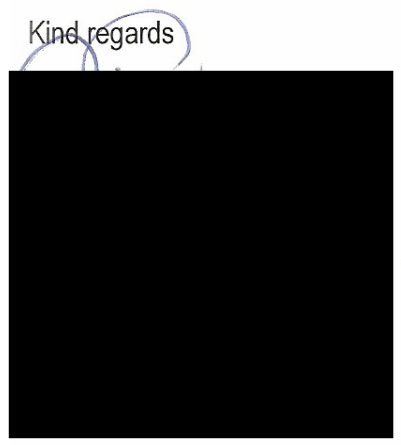




\title{
Appendix C: Information Sheet for Interview
}

\author{
VICTORIA UNTVERSTTYO OWEIITMGTON

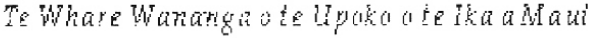

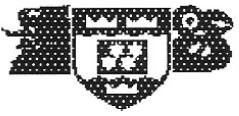

Information Sheet for Interview

Evidence into Practice: An evaluation of the implementation of customised birthweight centiles at one New Zealand DHB.

\section{Introduction:}

You are invited to take part in an evaluation study looking at the process of putting evidence into practice. In particular this study will look at the process of implementing customised birthweight centiles at your DHB. You have been identified as one of the key people involved in this process. Your participation is entirely voluntary and you may choose to withdraw from the study at any time.

This study has been granted ethical approval by the Northern Region Ethics Committee (Ethics Reference NTY/09/60/EXP) and has also been approved by I

Chairman of the hospital clinical board.

This study will be written up as part of a Masters in Midwifery degree by Malia Lardelli and is being supervised by Dr Joan Skinner, a senior lecturer in the Graduate School of Nursing, Midwifery and Health at Victoria University of Wellington.

\section{About the Study:}

\section{The Question}

How was the evidence behind the use of CBWC put into practice at one NZ DHB and what are the outcomes resulting from translating this knowledge into action?

Aims and objectives:

- To identify and critique the evidence relating to the use of CBWC

- To describe how the CBWC was implemented in one NZ DHB

- To evaluate the processes followed when utilising the CBWC

- To identify outcomes relating to the number of babies identified as SGA or LGA using CBWC as compared with other methods

Overall research design/methodology

This is a mixed methods evaluation study that uses both quantitative and qualitative methods. The methods include a literature review, interviews with key stakeholders, focus groups and an audit of practice.

\section{What is involved?}

- The interview is designed to take 30 minutes and can take place at a mutually agreed time and place. You will be interviewed by Malia Lardelli, the principal investigator. You will be required to sign the attached consent form prior to the interview. 
- Your participation is entirely voluntary. If you agree to take part in the interview you are free to withdraw at any time without having to give a reason and you do not have to answer all interview questions.

- You will be asked several semi-structured questions regarding your involvement in the implementation of customised birthweight centiles, including why and how this process was implemented.

- The interview will be tape-recorded, but not transcribed. The recording will be used to complement the notes taken during the interview.

- Your confidentiality will be maintained in any written reports as you will not be identified by name. Your job title instead may be used when relevant to discussing specific steps in the implementation process. The DHB will also remain unidentified.

- All raw data collected during the interview will be seen by the principal investigator and research supervisor only. These will be stored in a locked filing cabinet or as password protected electronic documents for 10 years and then deleted/wiped/shredded.

Please feel free to contact the researcher or research supervisor if you have any questions about this study.

\section{Principal Investigator:}

Malia Lardelli

TDH Midwife

Masters of Midwifery by Thesis student - Victoria University of Wellington

25 Griffiths Gr, Newlands, Wellington 6037

044776048 / 021912647

jm.lardelli@xtra.co.nz

\section{Research Supervisor:}

Dr Joan Skinner

Senior Lecturer - Victoria University of Wellington

PO Box 600, Wellington 6140

044636654

joan.skinner@vuw.ac.nz 


\section{Appendix D: Consent Form for Interview}

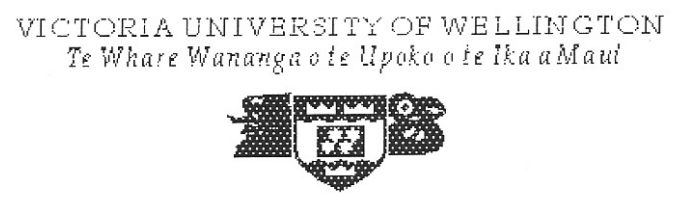

Consent Form for Interview

Evidence into Practice: An evaluation of the implementation of customised birthweight centiles at one New Zealand DHB.

- I have read and understood the attached 'Information sheet for Interview' dated $21 / 07 / 2009$ and I am satisfied that any questions I may have about participating in the interview have been answered.

- I agree to participate in this semi-structured interview and understand I may withdraw at any time without explanation and that I do not have to answer all questions.

- I understand that the interview will be tape recorded, but not transcribed, and that only the researcher and her supervisor will have access to the tape.

- I understand all data collected will be stored in a locked filing cabinet or as password protected documents and will then be deleted/wiped/shredded after 10 years.

- I understand that my name will not be used in any written reports. I understand my job title may be used when relevant to discussing specific steps in the implementation process. The DHB will also remain unidentified. by being interviewed.

(full name) hereby consent to take part in this study

Signature:

Date:

Interview conducted by:

Signature:

Date: 


\section{Appendix E: Interview Question Guide}

\section{Interview semi-structured question guide}

1) How did you first hear about customised birthweight centiles (CBWC)?

2) Why did you decide to start using CBWC at the unit?

3) What steps were involved in implementing the use of CBWC at your hospital?

4) What was your involvement in the implementation of this method?

5) What were some of the barriers in implementing the change to this method?

6) How was this change in policy accepted by members of staff?

7) How well do you feel the process is followed at the unit?

8) How well do you think staff understanding what a CBWC is?

9) What benefits, if any, have you noticed since the change to using CBWC?

10) What negative effects, if any, have you noticed since the change to using CBWC? 


\title{
Appendix F: Information Sheet for Focus Group
}

\author{
VICTORIA UNTVERSTY OF WELLINGTON

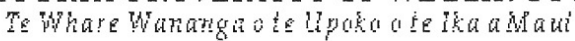

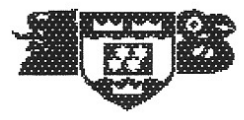 \\ Information Sheet for Focus Group \\ Evidence into Practice: An evaluation of the implementation of \\ customised birthweight centiles at one New Zealand DHB.
}

\section{Introduction:}

You are invited to take part in an evaluation study looking at the process of putting evidence into practice. In particular this study will look at the process of implementing customised birthweight centiles (CBWC) at your DHB and how this works in practice. You are invited to take part in a focus group which will discuss this topic. One focus group will be held with staff midwives and nurses and another will be held with Lead Maternity Caregivers (LMCs). Your participation is entirely voluntary and you may choose to withdraw from the study at any time.

This study has been granted ethical approval by the Northern Region Ethics Committee (Ethics Reference NTY/09/60/EXP) and has also been approved by D Chairman of the hospital clinical board.

This study will be written up as part of a Masters in Midwifery degree by Malia Lardelli and is being supervised by Dr Joan Skinner, a senior lecturer in the Graduate School of Nursing, Midwifery and Health at Victoria University of Wellington.

\section{About the Study:}

The Question

How was the evidence behind the use of CBWC put into practice at one NZ DHB and what are the outcomes resulting from translating this knowledge into action?

Aims and objectives:

- To identify and critique the evidence relating to the use of $\mathrm{CBWC}$

- To describe how the CBWC was implemented in one NZ DHB

- To evaluate the processes followed when utilising the CBWC

- To identify outcomes relating to the number of babies identified as SGA or LGA using CBWC as compared with other methods

Overall research design/methodology

This is a mixed methods evaluation study that uses both quantitative and qualitative methods. The methods include a literature review, interviews with key stakeholders, focus groups and an audit of practice. 


\title{
What is involved?
}

- The focus group is designed to take 45-60 minutes at a specified time and venue. It will be facilitated by Malia Lardelli, the principal investigator. You will be required to sign the attached consent form prior to participating in the focus group.

- Your participation is entirely voluntary. If you agree to take part in the focus group you are free to withdraw at any time without having to give a reason and you do not have to answer all questions.

- Participants will be asked to exhibit common courtesy towards others at all times by respecting all opinions and by agreeing to maintain the confidentiality of the group by not discussing individuals and their comments outside of the focus group.

- The group will be asked several semi-structured questions regarding the implementation and use of CBWC at the maternity unit and their role in putting them into practice and whether this process is working.

- The focus group discussion will be tape-recorded, but not transcribed. The recording will be used to complement the notes taken during the focus group by the principal investigator.

- All raw data collected during the focus groups will be accessed by the principal investigator and research supervisor only. These will be stored in a locked filing cabinet or as password protected electronic documents and deleted/wiped/shredded after 10 years.

- As a thank you for your time you will be provided with refreshments during the focus group.

Please feel free to contact the researcher or research supervisor if you have any questions about this study.

\author{
Principal Investigator: \\ Malia Lardelli \\ TDH Midwife \\ Masters of Midwifery by Thesis student - Victoria University of Wellington \\ 25 Griffiths Gr, Newlands, Wellington 6037 \\ 044776048 / 021912647 \\ jm.lardelli@xtra.co.nz \\ Research Supervisor: \\ Dr Joan Skinner \\ Senior Lecturer - Victoria University of Wellington \\ PO Box 600, Wellington 6140 \\ 044636654 \\ joan.skinner@vuw.ac.nz
}




\section{Appendix G: Consent Form for Focus Group}

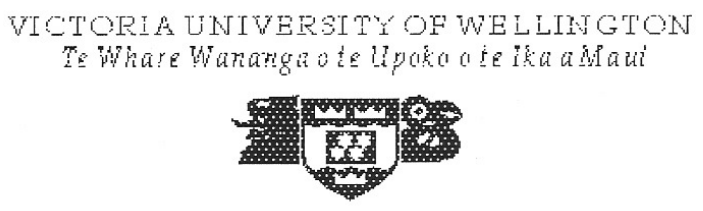

Consent Form for Focus Group

Evidence into Practice: An evaluation of the implementation of customised birthweight centiles at one New Zealand DHB.

- I have read and understood the attached 'Information sheet for Focus Group' dated 21/07/2009 and I am satisfied that any questions I may have about participating in the focus group has been answered.

- I agree to participate in this focus group and understand I may withdraw at any time without explanation and that I do not have to answer all questions.

- I agree to exhibit common courtesy towards others at all times and to maintain the confidentially of other participants.

- I understand that the focus group will be tape recorded, but not transcribed, and that only the researcher and her supervisor will have access to the tape.

- I understand all data collected will be stored in a locked filing cabinet or as password protected electronic documents and then destroyed after 10 years.

- I understand that no individual from the focus groups will be named in any written reports. The DHB will also remain unidentified.

I

by participating in this focus group.

(full name) hereby consent to take part in this study

Signature:

Date:

Focus Group conducted by:

Signature:

Date: 


\section{Appendix H: Invitation Poster for Focus Group}

VICTORIA UNTVERSTTY OF WELITMGTON

Te Whare Wananga o te Ipokn ate ika a Mau

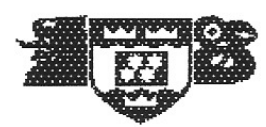

\section{FOCUS GROUP INVITATION}

To all LMCs and maternity unit midwives and nurses:

For my Masters Thesis in midwifery I am interested in evaluating how evidence is incorporated into practice.

You are invited to participate in a focus group where we will be discussing the implementation and use of customised birthweight centiles (CBWC) at the maternity unit.

Please find an information sheet in your cubby holes for more details.

\section{REFRESHMEDTS WILL BE PROVIDED!}

LMCs and staff midwives often have different roles in the process of implementing CBWCs, so will have separate discussions.

$$
\begin{gathered}
\text { Focus Group for LMCs: } \\
\text { Where } \\
\text { When }
\end{gathered}
$$

Focus Group for staff midwives/nurses:

Where

When

Please RSVP to: Malia Lardelli at 021912647 or jm.lardelli@xtra.co.nz

If you have any questions regarding this research you may contact myself or my research supervisor Dr Joan Skinner at 044636654 / joan.skinner@vuw.ac.nz

\section{I hope to see lots of you there!}




\section{Appendix I: Focus Group Question Guide LMCs}

1) How was the CBWC implemented at the maternity unit?

- What consultation did you receive prior to the implementation?

- What was your involvement in the implementation?

- How did this implementation take place?

- Why do you think it was implemented?

2) What is your role in using the CBWC?

- Collecting information eg height/weight of mother is needed antenatally, what issues are there around collecting this information?

- Once a baby is born what is your role in calculating the CBWC? Is this part of your role or the core staff's role?

- Before handing women over to core staff do you check the CBWC has been done and why/why not?

- What information is shared with women about the CBWC antenatally/postnatally?

3) What is the process in calculating and recording the CBWC?

- What issues are there around calculating the CBWC? Is easy/hard to do?

- How quickly is this calculation usually made?

- How well is the process followed at the unit, do all staff follow the same process?

- Where is the CBWC recorded?

- Is the process to follow clear in the guideline?

4) What are the positive and negative effects of using the CBWC?

- What benefits have you noticed since the CBWC was implemented?

- What negative effects have you noticed since the CBWC was implemented?

5) What is your understanding of the CBWC?

- What does a CBWC mean? How well do you think LMC's/staff understand it?

- How do you think the evidence does/doesn't support the use of CBWC? 


\section{Appendix J: Focus Group Question Guide Core Staff}

1) How was the CBWC implemented at the maternity unit?

- What consultation did you receive prior to the implementation?

- What was your involvement in the implementation?

- How did this implementation take place?

- Why do you think it was implemented?

2) What is your role in using the CBWC?

- What issues are there around the antenatal collection of information required to calculate the $\mathrm{CBWC}$ ?

- Once a baby is born what is your role in calculating the CBWC? Is this the LMCs role or your role?

- What information do you share with women about the CBWC postnatally?

3) What is the process in calculating and recording the CBWC?

- What issues are there around calculating the CBWC? Is easy/hard to do?

- How quickly is this calculation usually made?

- How well is the process followed at the unit, do all staff follow the same process?

- Where is the CBWC recorded?

- Is the process to follow clear in the guideline?

4) What are the positive and negative effects of using the CBWC?

- What benefits have you noticed since the CBWC was implemented?

- What negative effects have you noticed since the CBWC was implemented?

5) What is your understanding of the CBWC?

- What does a CBWC mean? How well do you think LMC's/staff understand it?

- How do you think the evidence does/doesn't support the use of CBWC? 


\section{Appendix K: Audit Tool}

\begin{tabular}{|c|c|c|c|c|c|c|c|c|c|c|c|c|c|c|c|c|c|c|c|}
\hline & 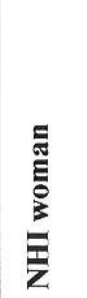 & 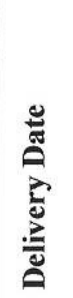 & 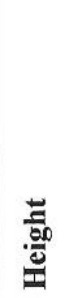 & 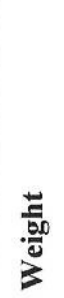 & $\sum_{\mu}^{\bar{n}}$ & $\begin{array}{l}\text { 总 } \\
\text { 咅 }\end{array}$ & 氖 & 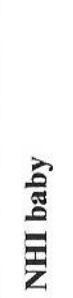 & 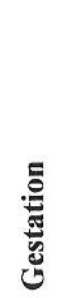 & ڤ̆ & $\begin{array}{l}\text { 总 } \\
\text { 童 }\end{array}$ & 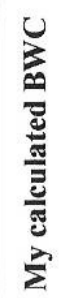 & 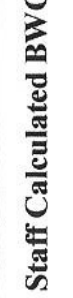 & 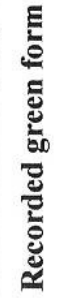 & 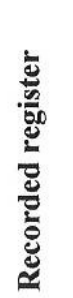 & 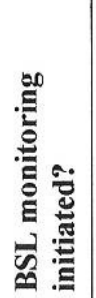 & 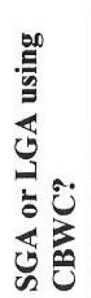 & 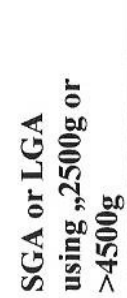 & 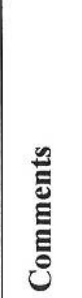 \\
\hline 1 & & & & & & & & & & & & & & & & & & & \\
\hline 2 & & & & & & & & & & & & & & & & & & & \\
\hline 3 & & & & & & & & & & & & & & & & & & & \\
\hline 4 & & & & & & & & & & & & & & & & & & & \\
\hline 5 & & & & & & & & & & & & & & & & & & & \\
\hline 6 & & & & & & & & & & & & & & & & & & & \\
\hline 7 & & & & & & & & & & & & & & & & & & & \\
\hline 8 & & & & & & & & & & & & & & & & & & & \\
\hline 9 & & & & & & & & & & & & & & & & & & & \\
\hline 10 & & & & & & & & & & & & & & & & & & & \\
\hline etc & & & & & & & & & & & & & & & & & & & \\
\hline
\end{tabular}




\section{Appendix L: Hypoglycaemia Flow Chart}

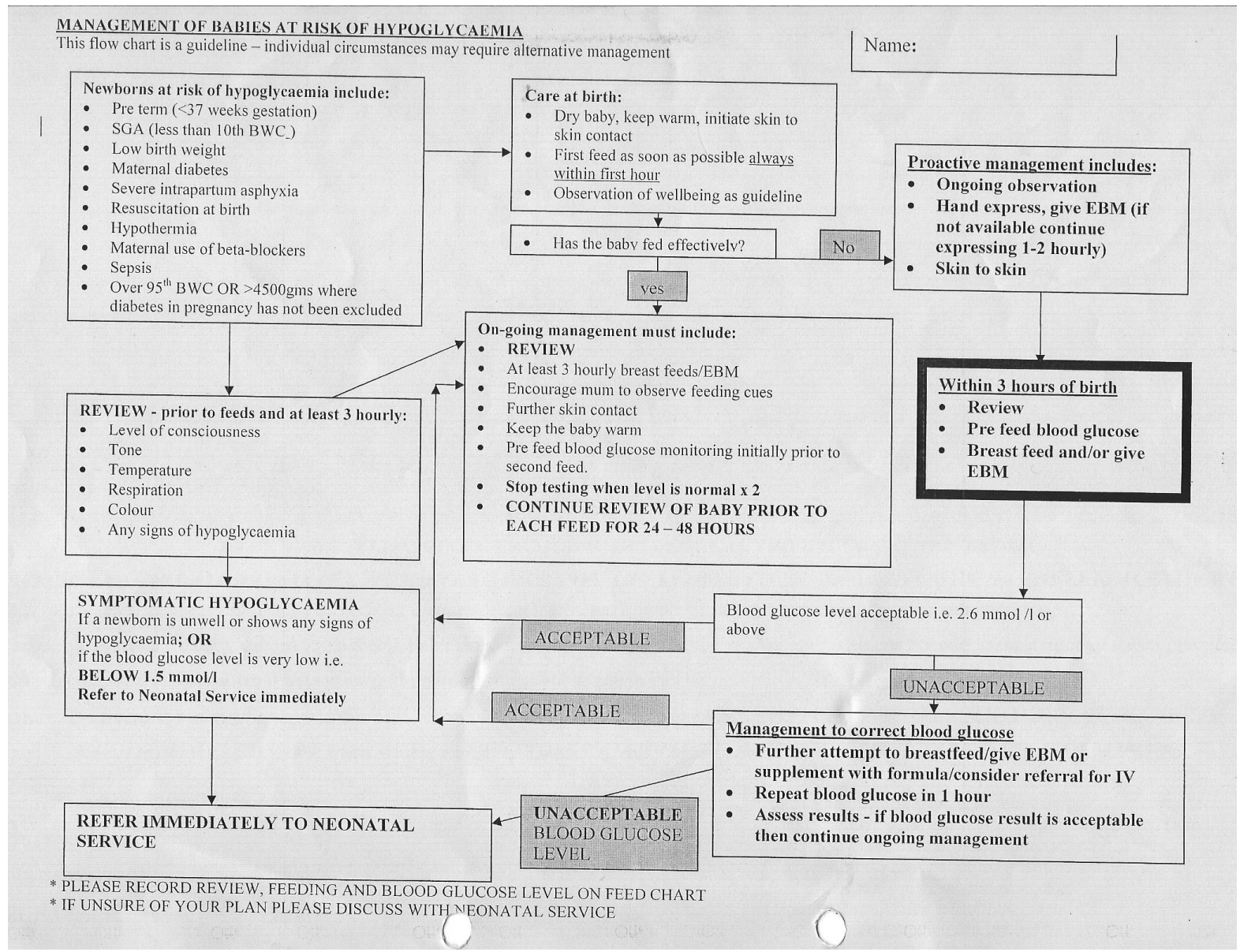




\section{Appendix M: Feedchart}

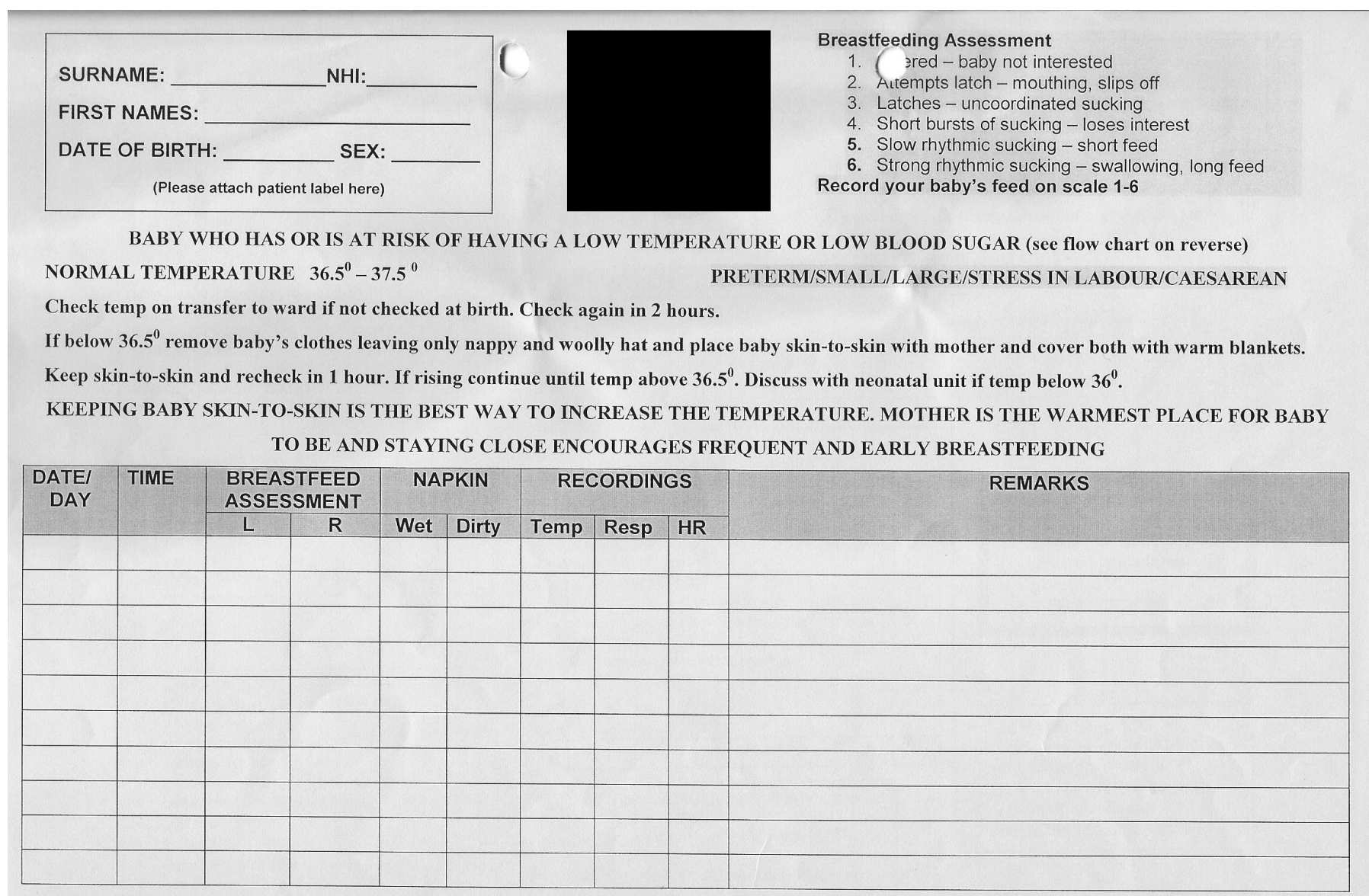

February 2009 


\section{Appendix N: Infant Summary Sheet}

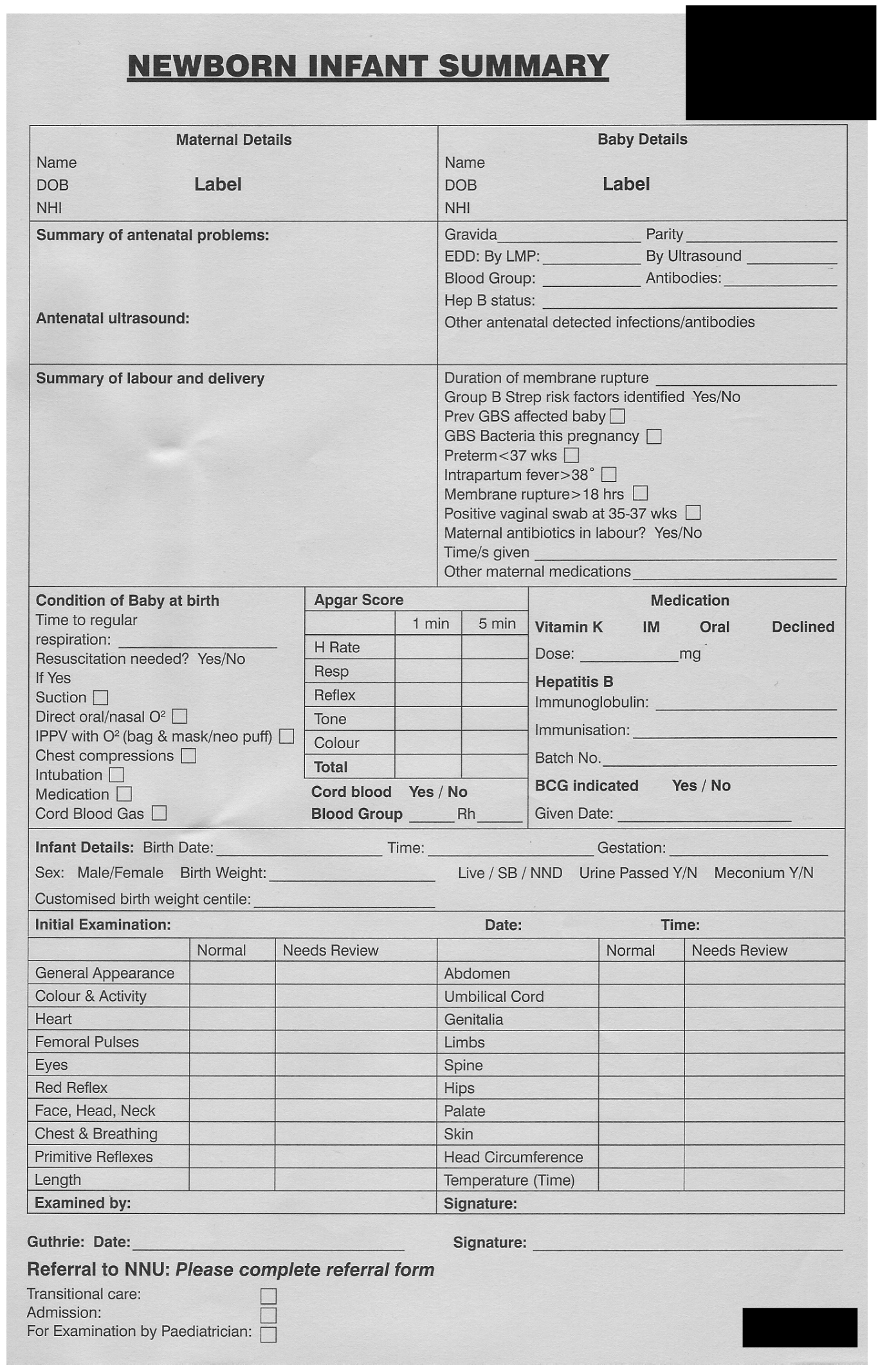




\section{Appendix O: Population Birthweight Centile Charts}

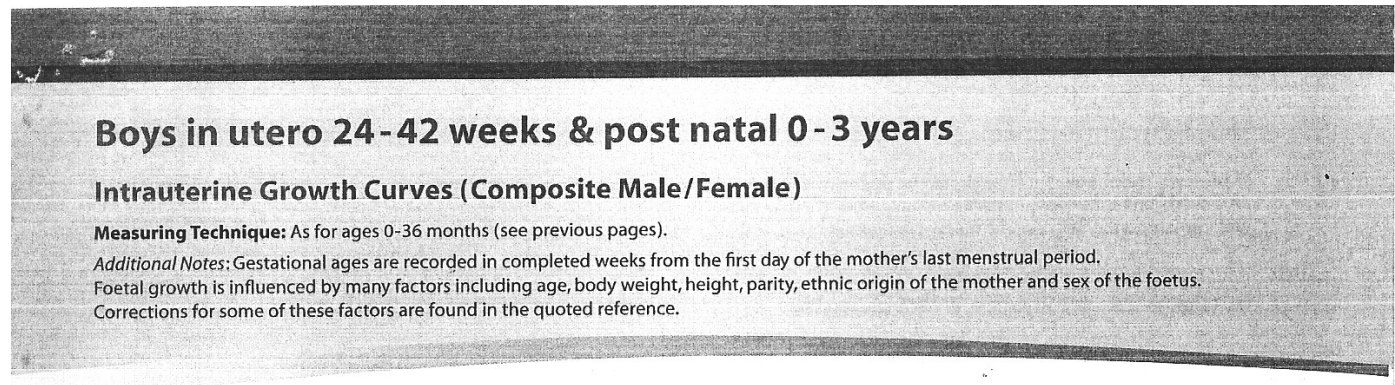

Data Source: Kitchen, W. H. et al. 1983,'Revised intrauterine growth curves for an Australian hospital population', Aust. Paediatr.J. 19:157-161.
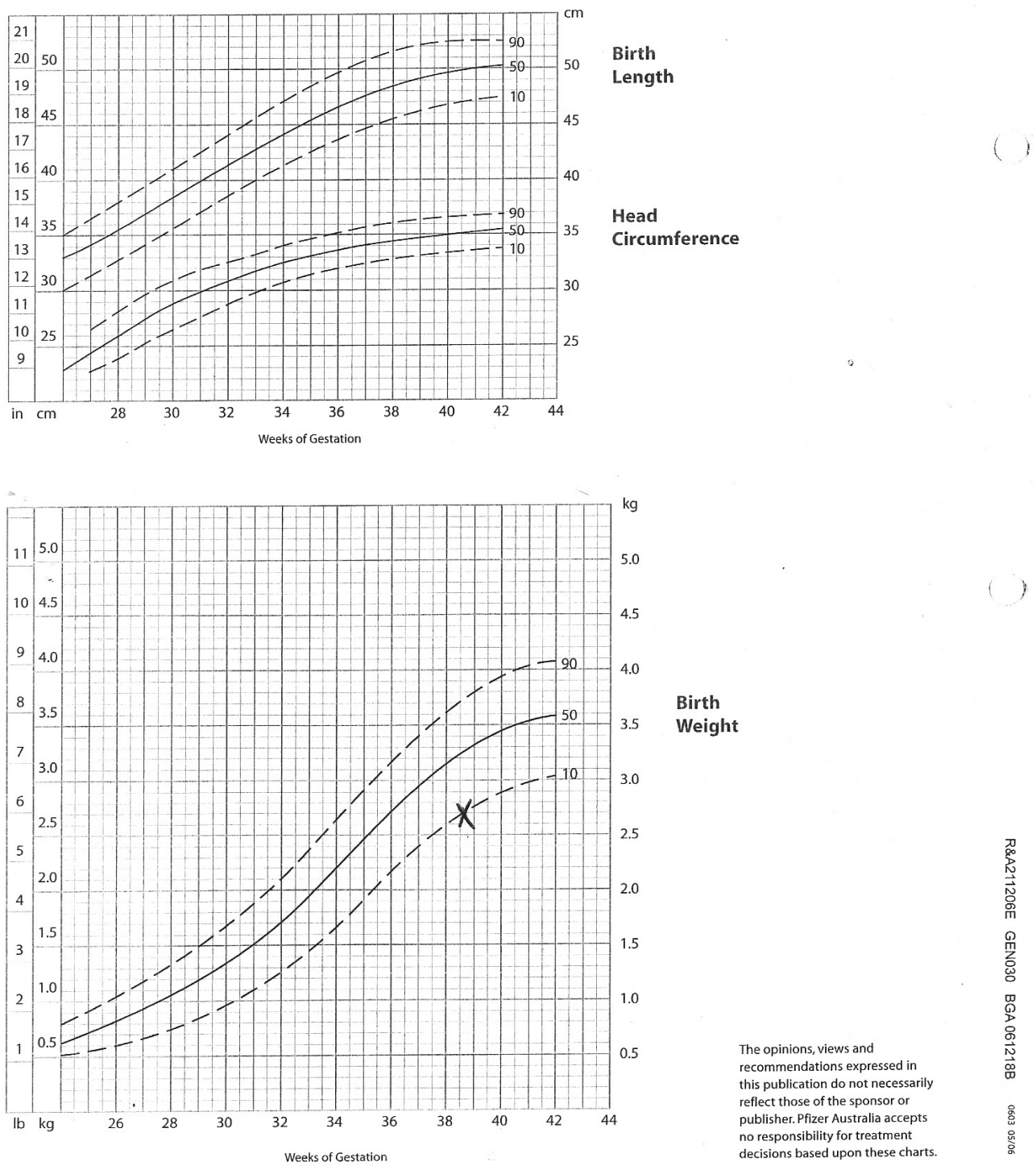


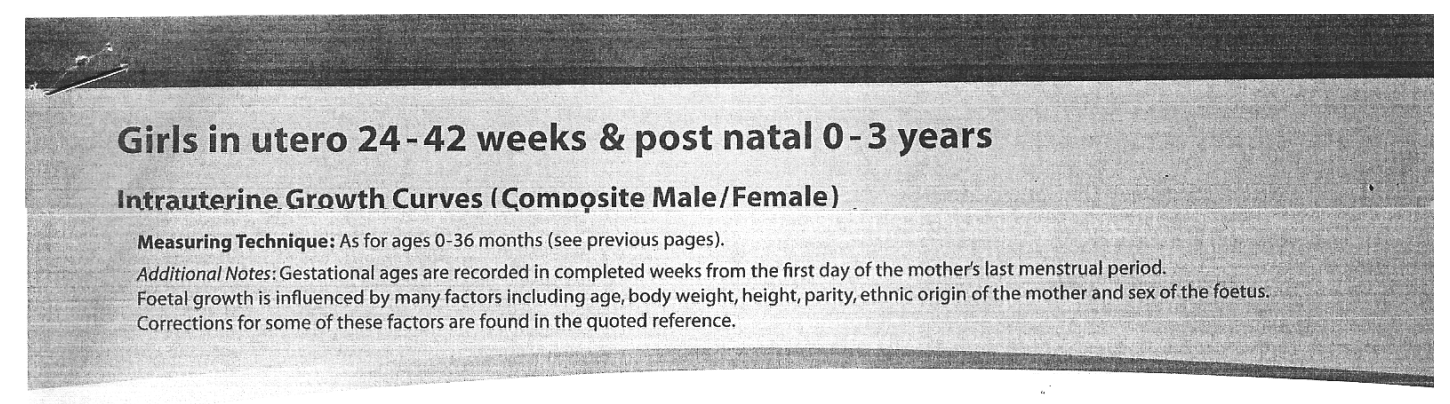

Data Source: Kitchen, W. H.et al. 1983, 'Revised intrauterine growth curves for an Australian hospital population', Aust. Paediatr.J.19:157-161.
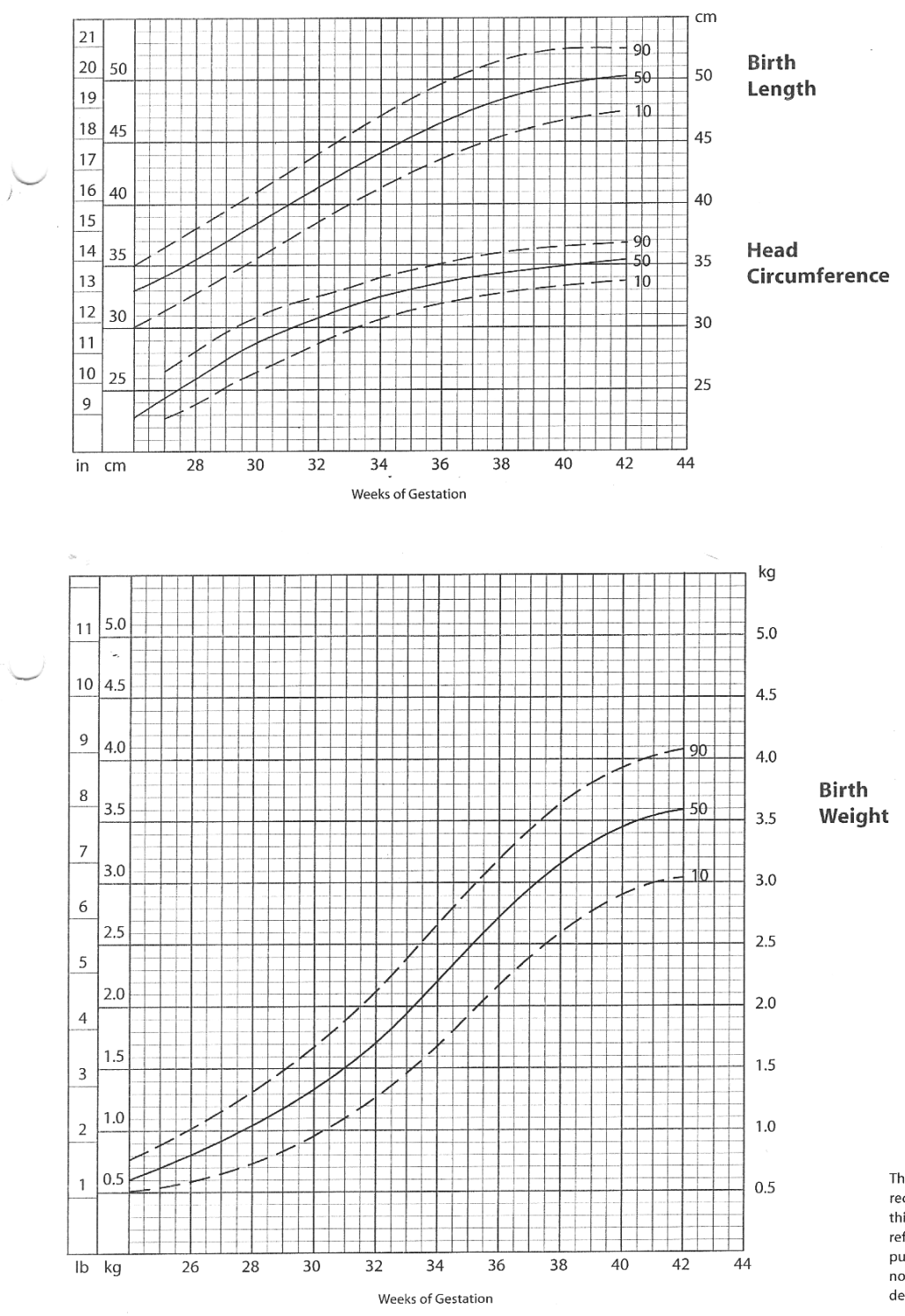

Birth
Weight 


\section{References}

Bakketeig, L. S., \& Magnus, P. (1992). Small-for-gestational-age (SGA) definitions and associated risks. International Journal of Technology Assessment in Health Care, 8 Suppl 1, 139-146. doi: 10.1017/ S0266462300013039

Barbour, R. (2008). Introducting qualitative research: A student guide to the craft of doing qualitative research. London: SAGE.

Battaglia, F. C., \& Lubchenco, L. O. (1967). A practical classification of newborn infants by weight and gestational age. The Journal of Pediatrics, 71(2), 159-163. doi: 10.1016/S0022-3476(67)80066-0

Beeby, P. J., Bhutap, T., \& Taylor, L. K. (1996). New South Wales populationbased birthweight percentile charts. Journal of Paediatrics and Child Health, 32(6), 512-518. doi: 10.1111/j.1440-1754.1996.tb00965.x

Boogaerts, M., Grealish, L., \& Ranse, K. (2008). Policy and practice: Exploring tensions to develop practice. Practice Development in Health Care, 7(1), 49-57. doi: 10.1002/pdh.240

Braun, V., \& Clarke, V. (2006). Using thematic analysis in psychology. Qualitative Research in Psychology, 3(2), 77-101. doi: 10.1191/ 1478088706qp063oa

Brophy, S., Snooks, H., \& Griffiths, L. (2008). Small-scale evaluation in health: A practical guide. London: SAGE.

Brouwers, M., Stacey, D., \& O'Connor, A. (2010). Knowledge creation: Synthesis, tools and products. Canadian Medical Association Journal 182(2), E68-72. doi: 10.1503/cmaj.081230

Campbell, M., Fitzpatrick, R., Haines, A., Kinmonth, A. L., Sandercock, P., Spiegelhalter, D., \& Tyrer, P. (2000). Framework for design and evaluation of complex interventions to improve health. BMJ, 321(7262), 694-696. doi: 10.1136/bmj.321.7262.694

Carmines, E. G., \& Zeller, R. A. (1979). Reliability and validity assessment. California: SAGE.

Carter, B. (2004). How do you analyse qualitative data? In T. Lavender, G. Edwards \& Z. Alfirevic (Eds.), Demystifying qualitative research in pregnancy and childbirth (pp. 87-108). Wiltshire: Quay Books.

Child and Youth Mortality Review Committee. (2009). Fifth report to the minister of health: Reporting mortality 2002-2008. Wellington: Child and Youth Mortality Review Committee. 
Clausson, B., Gardosi, J., Francis, A., \& Cnattingius, S. (2001). Perinatal outcome in SGA births defined by customised versus population-based birthweight standards. BJOG : An International Journal of Obstetrics and Gynaecology, 108(8), 830-834. doi: 10.1111/j.1471-0528.2001.00205.x

Cnattingius, S., Bergstrom, R., Lipworth, L., \& Kramer, M. S. (1998).

Prepregnancy weight and the risk of adverse pregnancy outcomes. The New England Journal of Medicine, 338(3), 147-152. doi: 10.1056/ NEJM199801153380302

Cornblath, M., Hawdon, J. M., Williams, A. F., Aynsley-Green, A., Ward-Platt, M. P., Schwartz, R., \& Kalhan, S. C. (2000). Controversies regarding definition of neonatal hypoglycemia: Suggested operational thresholds. Pediatrics, 105(5), 1141-1145. doi: 10.1542/peds.105.5.1141

Cowan, J. (2009, December). Customise growth charts and birth weight centiles. Midwifery News, 30-31.

Creswell, J. W. (2009). Research design: Qualitative, quantitative, and mixed methods approaches (3rd ed.). California: SAGE.

Datta, L. (2001). The wheelbarrow, the mosaic and the double helix: Challenges and strategies for successfully carrying out mixed methods evaluation. Evaluation Journal of Australasia, 1(2). Retrieved September 2, 2010, from http://www.aes.asn.au/publications/vol1no2/wheelbarrow_mosaic_ and_double_helix.pdf

Davies, B., \& Edwards, N. (2009). Sustaining knowledge use. In S. Straus, J. Tetroe \& I. Graham (Eds.), Knowledge translation in health care: Moving from evidence to practice (pp. 165-173). Oxford: Blackwell Publishing.

Davis, D., \& Davis, N. (2010). Selecting educational interventions for knowledge translation. Canadian Medical Association Journal, 182(2), E89-93. doi: 10.1503/cmaj.081241

De Jong, C. L. D., Gardosi, J., Dekker, G. A., Colenbrander, G. J., \& Van Geijn, H. P. (1998). Application of a customised birthweight standard in the assessment of perinatal outcome in a high risk population. BJOG: An International Journal of Obstetrics \& Gynaecology, 105(5), 531-535. doi: 10.1111/j.1471-0528.1998.tb10154.x

Doran, D. M. (2010). An outcomes framework for knowledge translation. In D. Bick \& I. Graham (Eds.), Evaluating the impact of implementing evidence-based practice (pp. 67-85). Chichester: Wiley-Blackwell. 
Ego, A., Subtil, D., Grange, G., Thiebaugeorges, O., Senat, M. V., Vayssiere, C., \& Zeitlin, J. (2006). Customized versus population-based birth weight standards for identifying growth restricted infants: A french multicenter study. American Journal of Obstetrics and Gynecology, 194(4), 10421049. doi: 10.1016/j.ajog.2005.10.816

Ego, A., Subtil, D., Grange, G., Thiebaugeorges, O., Senat, M. V., Vayssiere, C., \& Zeitlin, J. (2008). Should parity be included in customised fetal weight standards for identifying small-for-gestational-age babies? Results from a french multicentre study. BJOG : An International Journal of Obstetrics and Gynaecology, 115(10), 1256-1264. doi: 10.1111/j.14710528.2008.01855.x

Ferlie, E. (2005). From evidence to actionable knowledge? In S. Dopson \& L. Fitzgerald (Eds.), Knowledge to action? Evidence-based health care in context (pp. 182-197). Oxford: Oxford University Press.

Ferlie, E., \& Dopson, S. (2005). Studying complex organizations in health care. In S. Dopson \& L. Fitzgerald (Eds.), Knowledge to action? Evidencebased health care in context (pp. 8-27). Oxford: Oxford University Press.

Fitzgerald, L., \& Dopson, S. (2005). Knowledge, credible evidence, and utilization. In S. Dopson \& L. Fitzgerald (Eds.), Knowledge to action? Evidence-based health care in context (pp. 132-154). Oxford: Oxford University Press.

Fitzgerald, L., Dopson, S., Ferlie, E., \& Locock, L. (2005). Knowledge in action. In S. Dopson \& L. Fitzgerald (Eds.), Knowledge to action? Evidencebased health care in context (pp. 155-181). Oxford: Oxford University Press.

Foy, R., \& Eccles, M. P. (2009). Audit and feedback interventions. In S. Straus, J. Tetroe \& I. Graham (Eds.), Knowledge translation in health care: Moving from evidence to practice (pp. 126-131). Oxford: Blackwell Publishing.

Gardosi, J. (2006). New definition of small for gestational age based on fetal growth potential. Hormone Research, 65 Suppl 3, 15-18. doi: 10.1159/ 000091501

Gardosi, J. (2009a). Intrauterine growth restriction: New standards for assessing adverse outcome. Best Practice \& Research Clinical Obstetrics \& Gynaecology, 23(6), 741-749. doi: 10.1016/j.bpobgyn.2009.09.001

Gardosi, J. (2009b). This document describes the application of the customised growth potential to assess fetal size and growth, using the Gestation Related Optimal Weight (GROW) software. Retrieved August 11, 2010, from http://www.gestation.net/GROW\%20doc\%20Aug09.pdf 
Gardosi, J., Chang, A., Kalyan, B., Sahota, D., \& Symonds, E. M. (1992).

Customised antenatal growth charts. Lancet, 339(8788), 283-287. doi:

10.1016/0140-6736(92)91342-6

Gardosi, J., Clausson, B., \& Francis, A. (2007). The use of customised versus population-based birthweight standards in predicting perinatal mortality. BJOG : An International Journal of Obstetrics and Gynaecology, 114(10), 1301-1302; author reply 1303. doi: 10.1111/j.14710528.2007.01432.x

Gardosi, J., Clausson, B., \& Francis, A. (2009). The value of customised centiles in assessing perinatal mortality risk associated with parity and maternal size. BJOG : An International Journal of Obstetrics and Gynaecology, 116(10), 1356-1363. doi: 10.1111/j.1471-0528.2009.02245.x

Gardosi, J., \& Francis, A. (2009a). Adverse pregnancy outcome and association with small for gestational age birthweight by customized and populationbased percentiles. American Journal of Obstetrics and Gynecology, 201(1), 28 e21-28. doi: 10.1016/j.ajog.2009.04.034

Gardosi, J., \& Francis, A. (2009b). A customized standard to assess fetal growth in a US population. American Journal of Obstetrics and Gynecology, 201(1), 25 e21-27. doi: 10.1016/j.ajog.2009.04.035

Gardosi, J., \& Francis, A. (2009c). Parity and smallness for gestational age. BJOG: An International Journal of Obstetrics \& Gynaecology, 116(8), 1135-1136. doi: 10.1111/j.1471-0528.2009.02127.x

Gardosi, J., Mongelli, J. M., \& Mul, T. (1995). Intrauterine growth retardation. Bailliere's Clinical Obstetrics and Gynaecology, 9(3), 445-463. doi: 10.1016/S0950-3552(05)80374-8

Gardosi, J., \& Mongelli, M. (1993). Risk assessment adjusted for gestational age in maternal serum screening for down's syndrome. BMJ, 306(6891), 1509-1511. doi: 10.1136/bmj.306.6891.1509

Gardosi, J., Mongelli, M., Wilcox, M., \& Chang, A. (1995). An adjustable fetal weight standard. Ultrasound in Obstetrics \& Gynecology, 6(3), 168-174. doi: 10.1046/j.1469-0705.1995.06030168.x

GlaxoSmithKline NZ Ltd. (2010). Data sheet: Aropax tablets paroxetine. Retrieved August 1, 2011, from http://www.medsafe.govt.nz/profs /datasheet/a/Aropaxtab.pdf

Goldenberg, R. L., \& Cliver, S. P. (1997). Small for gestational age and intrauterine growth restriction: Definitions and standards. Clinical Obstetrics and Gynecology, 40(4), 704-714. 
Graham, I., Bick, D., Tetroe, J., Straus, S., \& Harrison, M. (2010). Measuring outcomes of evidence-based practice: Distinguishing between knowledge use and its impact. In D. Bick \& I. Graham (Eds.), Evaluating the impact of implementing evidence-based practice (pp. 18-37). Chichester: WileyBlackwell.

Graham, I., Logan, J., Harrison, M. B., Straus, S., Tetroe, J., Caswell, W., \& Robinson, N. (2006). Lost in knowledge translation: Time for a map? The Journal of Continuing Education in the Health Professions, 26(1), 13-24. doi: $10.1002 /$ chp.47

Graham, I., \& Tetroe, J. (2007). Some theoretical underpinnings of knowledge translation. Academic Emergency Medicine, 14(11), 936-941. doi: 10.1197/j.aem.2007.07.004

Graham, I., \& Tetroe, J. (2009). Implementation of evidence. International Journal of Evidence-Based Healthcare, 7(3), 157-158. doi: 10.1111/j.1744-1609.2009.00140.x

Graham, I., \& Tetroe, J. (2010). The knowledge to action framework. In J. Rycroft-Malone \& T. Bucknall (Eds.), Models and frameworks for implementing evidence-based practice: Linking evidence to action (pp. 207-221). Chichester: Wiley-Blackwell.

Groom, K. M., Poppe, K. K., North, R. A., \& McCowan, L. (2007). Small-forgestational-age infants classified by customized or population birthweight centiles: Impact of gestational age at delivery. American Journal of Obstetrics and Gynecology, 197(3), 239 e231-235. doi:

10.1016/j.ajog.2007.06.038

Grundy, M. F., Hood, J., \& Newman, G. B. (1978). Birth weight standards in a community of mixed racial origin. British Journal of Obstetrics and Gynaecology, 85(7), 481-486. doi: 10.1111/j.1471-0528.1978.tb15618.x

Hakkennes, S., \& Green, S. (2006). Measures for assessing practice change in medical practitioners. Implementation Science 1, 29. doi: 10.1186/17485908-1-29

Halliday, H. L. (2009). Neonatal management and long-term sequelae. Best Practice \& Research Clinical Obstetrics \& Gynaecology, 23(6), 871-880. doi: 10.1016/j.bpobgyn.2009.06.005

Hammersley, M. (2008). Assessing validity in social research. In P. Alasuutari, L. Bickman \& J. Brannen (Eds.), The SAGE handbook of social research methods (pp. 42-53). London: SAGE.

Hanberg, A., \& Brown, S. C. (2006). Bridging the theory-practice gap with evidence-based practice. Journal of Continuing Education in Nursing, 37(6), 248-249. 
Harrison, M., Graham, I., \& Fervers, B. (2009). Adapting knowledge to local context. In S. Straus, J. Tetroe \& I. Graham (Eds.), Knowledge translation in health care: Moving from evidence to practice (pp. 73-82). Oxford: Blackwell Publishing.

Harrison, M., Legare, F., Graham, I., \& Fervers, B. (2010). Adapting clinical practice guidelines to local context and assessing barriers to their use. Canadian Medical Association Journal, 182(2), E78-84. doi: 10.1503/cmaj.081232

Health and Disability Commissioner. (2007). A report by the health and disability commissioner: Case 05HDC16723. Retrieved February 5, 2009, from http://www.hdc.org.nz/media/14764/05hdc16723midwife.pdf

Health Research Council of New Zealand. (2010). Diabetes in pregnancy 'creating dangerous cycle'. Retrieved September 1, 2011, from http://www.hrc.govt.nz/sites/default/files/Dr\%20Janet\%20Rowan\%20and \%20Prof\%20Elaine\%20Rush_0.pdf

Heiskanen, N., Raatikainen, K., \& Heinonen, S. (2006). Fetal macrosomia - a continuing obstetric challenge. Biology of the Neonate, 90(2), 98-103. doi: $10.1159 / 000092042$

Hennink, M., Hutter, I., \& Bailey, A. (2011). Qualitative research methods. London: SAGE.

Holtrop, P. C. (1993). The frequency of hypoglycemia in full-term large and small for gestational age newborns. American Journal of Perinatology, 10(2), 150-154. doi: 10.1055/s-2007-994649

Hutcheon, J. A., Zhang, X., Cnattingius, S., Kramer, M. S., \& Platt, R. W. (2008). Customised birthweight percentiles: Does adjusting for maternal characteristics matter? BJOG : An International Journal of Obstetrics and Gynaecology, 115(11), 1397-1404. doi: 10.1111/j.14710528.2008.01870.x

Johnson, B., \& Christensen, L. (2012). Educational research: Quantitative, qualitative, mixed approaches (4th ed.). California: SAGE.

Johnson, R., Onwuegbuzie, A. J., \& Turner, L. A. (2007). Toward a definition of mixed methods research. Journal of Mixed Methods Research, 1(2), 112133. doi: $10.1177 / 1558689806298224$

Kirk, J., \& Miller, M. L. (1986). Reliability and validity in qualitative research. California: SAGE.

Kitson, A., \& Phil, D. (2009). Knowledge translation and guidelines: A transfer, translation or transformation process? International Journal of EvidenceBased Healthcare, 7(2), 124-139. doi: 10.1111/j.1744-1609.2009.00130.x 
Landry, R., Amara, N., Pablos-Mendes, A., Shademani, R., \& Gold, I. (2006). The knowledge-value chain: A conceptual framework for knowledge translation in health. Bulletin of the World Health Organization, 84(8), 597-602. doi: 10.1590/S0042-96862006000800009

Larson, E. L., Bryan, J. L., Adler, L. M., \& Blane, C. (1997). A multifaceted approach to changing handwashing behavior. American Journal of Infection Control, 25(1), 3-10. doi: 10.1016/S0196-6553(97)90046-8

Maher, L., \& Evans, A. (n.d). Sustainability model and guide. Retrieved May 10, 2011, from http://www.evidenceintopractice.scot.nhs.uk/media/ 135265/sustainability_model.pdf

Majumdar, S. R. (2009). Case examples. In S. Straus, J. Tetroe \& I. Graham (Eds.), Knowledge translation in health care: Moving from evidence to practice (pp. 174-181). Oxford: Blackwell Publishing.

Mathison, S. (Ed.). (2005). Encyclopedia of evaluation. California: SAGE.

Maulik, D. (2006). Fetal growth compromise: Definitions, standards, and classification. Clinical Obstetrics and Gynecology, 49(2), 214-218.

McCowan, L., Harding, J. E., \& Stewart, A. (2005). Customized birthweight centiles predict SGA pregnancies with perinatal morbidity. BJOG : An International Journal of Obstetrics and Gynaecology, 112(8), 1026-1033. doi: 10.1111/j.1471-0528.2005.00656.x

McCowan, L., \& Stewart, A. (2004). Term birthweight centiles for babies from New Zealand's main ethnic groups. The Australian \& New Zealand Journal of Obstetrics \& Gynaecology, 44(5), 432-435. doi: 10.1111/j.1479-828X.2004.00273.X

McCowan, L., Stewart, A., Francis, A., \& Gardosi, J. (2004). A customised birthweight centile calculator developed for a New Zealand population. The Australian \& New Zealand Journal of Obstetrics \& Gynaecology, 44(5), 428-431. doi: 10.1111/j.1479-828X.2004.00272.x

Melnyk, B. M., Fineout-Overholt, E., Stone, P., \& Ackerman, M. (2000). Evidence-based practice: The past, the present, and recommendations for the millennium. Pediatric Nursing, 26(1), 77-80.

Mertens, D. M. (2005). Research and evaluation in education and psychology: Integrating diversity with quantitative, qualitative, and mixed methods (2nd ed.). California: SAGE.

Mertens, D. M. (2010). Research and evaluation in education and psychology: Integrating diversity with quantitative, qualitative, and mixed methods (3rd ed.). California: SAGE. 
Ministry of Health. (2002). Toward clinical excellence: An introduction to clinical audit, peer review and other clinical improvement activities. Wellington: Ministry of Health.

Ministry of Health. (2008). A portrait of health: Key results of the 2006/07 New Zealand health survey. Wellington: Ministry of Health.

Ministry of Health. (2010). Hospital based maternity events 2006. Wellington: Ministry of Health.

Ministry of Health, C. T. R. U. (2009). Clinical guidelines for weight management in New Zealand adults. Wellington: Ministry of Health.

Mitton, C., Adair, C. E., McKenzie, E., Patten, S. B., \& Perry, B. W. (2007). Knowledge transfer and exchange: Review and synthesis of the literature. The Milbank Quarterly, 85(4), 729-768. doi: 10.1111/j.14680009.2007.00506.x

Mongelli, M., Figueras, F., Francis, A., \& Gardosi, J. (2007). A customized birthweight centile calculator developed for an Australian population. The Australian \& New Zealand Journal of Obstetrics \& Gynaecology, 47(2), 128-131. doi: 10.1111/j.1479-828X.2007.00698.x

Mongelli, M., Wilcox, M., \& Gardosi, J. (1996). Estimating the date of confinement: Ultrasonographic biometry versus certain menstrual dates. American Journal of Obstetrics and Gynecology, 174(1 Pt 1), 278-281. doi: 10.1016/S0002-9378(96)70408-8

New Zealand Health Information Service. (2007). Report on maternity: Maternal and newborn information 2004. Wellington: Ministry of Health.

Oakley, J. R., \& Parsons, R. J. (1977). Skinfold thickness as an indicator of neonatal hypoglycaemia in infants with birthweights over $2500 \mathrm{~g}$. Developmental Medicine and Child Neurology, 19(5), 585-588. doi: 10.1111/j.1469-8749.1977.tb07990.x

Onwuegbuzie, A. J., \& Johnson, R. B. (2006). The validity issue in mixed research. Research in the Schools, 13(1). Retrieved August 1, 2011, from http://carbon.videolectures.net/v005/e1/4gi2nosqk7a4u3rhmb6f4yl2huqff 7a5.pdf

Owen, J. M. (2006). Program evaluation: Forms and approaches (3rd ed.). New York: The Guilford Press.

Patton, M. Q. (2002). Qualitative research and evaluation methods. California: SAGE.

Patton, M. Q. (2008). Utilization-focused evaluation (4th ed.). Los Angeles: SAGE. 
PMMRC. (2009). Perinatal and maternal mortality in New Zealand 2006:

Second report to the minister of health June 2007 to June 2008.

Wellington: Ministry of Health.

PMMRC. (2011). Fifth annual report of the perinatal and maternal mortality review committee: Reporting mortality 2009. Wellington: Health and Quality \& Safety Commission.

Resnik, R. (2007). One size does not fit all. American Journal of Obstetrics and Gynecology, 197(3), 221-222. doi: 10.1016/j.ajog.2007.07.019

Rogers, E. M. (2005). Diffusion of innovations (5th ed.). New York: Free Press.

Rozance, P. J., \& Hay, W. W. (2006). Hypoglycemia in newborn infants: Features associated with adverse outcomes. Biology of the Neonate, 90(2), 74-86. doi: 10.1159/000091948

Rycroft-Malone, J., \& Bucknall, T. (2010a). Summary and concluding comments. In J. Rycroft-Malone \& T. Bucknall (Eds.), Models and frameworks for implementing evidence-based practice: Linking evidence to action (pp. 247-257). Chichester: Wiley-Blackwell.

Rycroft-Malone, J., \& Bucknall, T. (2010b). Theory, frameworks and models: Laying down the ground work. In J. Rycroft-Malone \& T. Bucknall (Eds.), Models and frameworks for implementing evidence-based practice: Linking evidence to action (pp. 23-50). Chichester: WileyBlackwell.

Saks, M., \& Allsop, J. (2008). Researching health: Qualitative, quantatitive and mixed methods. London: SAGE.

Schaefer-Graf, U. M., Rossi, R., Buhrer, C., Siebert, G., Kjos, S. L., Dudenhausen, J. W., \& Vetter, K. (2002). Rate and risk factors of hypoglycemia in large-for-gestational age newborn infants of nondiabetic mothers. American Journal of Obstetrics and Gynecology, 187(4), 913917. doi: 10.1067/mob.2002.126962

Sloan, C. T., \& Lorenz, R. P. (1991). Importance of locally derived birth weight nomograms. The Journal of Reproductive Medicine, 36(8), 598-602.

Smithson, J. (2008). Focus groups. In P. Alasuutari, L. Bickman \& J. Brannen (Eds.), The SAGE handbook of social research methods (pp. 357-370). London: SAGE.

Spyridonidis, D., \& Calnan, M. (2011). Opening the black box: A study of the process of NICE guidelines implementation. Health Policy, 102(2-3), 117-125. doi: 10.1016/j.healthpol.2011.06.011 
Stewart, A., Jackson, R. T., Ford, M. A., \& Beaglehole, R. (1987).

Underestimation of relative weight by use of self-reported height and weight. American Journal of Epidemiology, 125(1), 122-126.

Straus, S. (2009). The action cycle. In S. Straus, J. Tetroe \& I. Graham (Eds.), Knowledge translation in health care: Moving from evidence to practice (pp. 59-72). Oxford: Oxford University Press.

Straus, S., Graham, I., Taylor, M., \& Lockyer, J. (2008). Development of a mentorship strategy: A knowledge translation case study. The Journal of Continuing Education in the Health Professions, 28(3), 117-122. doi: 10.1002/chp.179

Straus, S., \& Holroyd-Leduc, J. (2008). Knowledge-to-action cycle. EvidenceBased Medicine, 13(4), 98-100. doi: 10.1136/ebm.13.4.98-a

Straus, S., Tetroe, J., \& Graham, I. (2009). Defining knowledge translation. Canadian Medical Association Journal 181(3-4), 165-168. doi: 10.1503/cmaj.081229

Straus, S., Tetroe, J., Graham, I., Zwarenstein, M., \& Bhattacharyya, O. (2009). Monitoring knowledge use and evaluating outcomes of knowledge use. In S. Straus, J. Tetroe \& I. Graham (Eds.), Knowledge translation in health care: Moving from evidence to practice (pp. 151-159). Oxford: Blackwell.

Straus, S., Tetroe, J., Graham, I., Zwarenstein, M., Bhattacharyya, O., \& Shepperd, S. (2010). Monitoring use of knowledge and evaluating outcomes. Canadian Medical Association Journal 182(2), E94-98. doi: 10.1503/cmaj.081335

Sudsawad, P. (2007). Knowledge translation: Introduction to models, strategies, and measures. Retrieved April 5, 2011, from http://www.ncddr.org/kt/ products/ktintro/ktintro.pdf

The Quit Group and the Ministry of Health. (2009). Maori smoking and tobacco use. Wellington: Ministry of Health.

Thompson, J. M., Mitchell, E. A., \& Borman, B. (1994). Sex specific birthweight percentiles by gestational age for New Zealand. The New Zealand Medical Journal, 107(970), 1-3.

UNICEF. (2008). Guidance on the development of policies and guidelines for the prevention and management of hypoglycaemia of the newborn. Retrieved February 5, 2009, from http://www.unicef.org.uk/Documents/ Baby_Friendly/Guidance/4/hypo_policy.pdf?epslanguage=en 
Voorhorst, F. J., Bouter, L. M., Bezemer, P. D., \& Kurver, P. H. (1993). Maternal characteristics and expected birth weight. European Journal of Obstetrics, Gynecology, and Reproductive Biology, 50(2), 115-122. doi: 10.1016/0028-2243(93)90175-C

Wensing, M., Bosch, M., \& Grol, R. (2010). Developing and selecting interventions for translating knowledge to action. Canadian Medical Association Journal 182(2), E85-88. doi: 10.1503/cmaj.081233

Wight, N., Marinelli, A., \& The Academy of Breastfeeding Medicine Protocol Committee. (2006). ABM clinical protocol \#1: Guidelines for glucose monitoring and treatment of hypoglycaemia in breastfed neonates. Breastfeeding Medicine, 1(3). doi: 10.1089/bfm.2006.1.178

Wollschlaeger, K., Nieder, J., Koppe, I., \& Hartlein, K. (1999). A study of fetal macrosomia. Archives of Gynecology and Obstetrics, 263(1-2), 51-55. doi: $10.1007 / \mathrm{s} 004040050262$

World Health Organization. (1961). Public health aspects of low birth weight: Third report of the expert committee on maternal and child health. Geneva: World Health Organization.

World Health Organization. (1997). Hypoglycaemia of the newborn: Review of the literature. Retrieved Feb 2, 2009, from http://whqlibdoc.who.int/ hq/1997/WHO_CHD_97.1.pdf

World Health Organization. (2006). Bridging the "Know -Do" gap meeting on knowledge translation in global health. Retrieved August 30, 2010, from http://www.who.int/kms/WHO_EIP_KMS_2006_2.pdf

Yu, C. K., Teoh, T. G., \& Robinson, S. (2006). Obesity in pregnancy. BJOG : An International Journal of Obstetrics and Gynaecology, 113(10), 11171125. doi: 10.1111/j.1471-0528.2006.00991.x

Zhang, X., Platt, R. W., Cnattingius, S., Joseph, K. S., \& Kramer, M. S. (2007). The use of customised versus population-based birthweight standards in predicting perinatal mortality. BJOG : An International Journal of Obstetrics and Gynaecology, 114(4), 474-477. doi: 10.1111/j.14710528.2007.01273.x 